Supporting Information

\title{
Lewis Acid Activated Reactions of Silyl Ketenes for the Preparation of $\alpha$-Silyl Carbonyl Compounds
}

\author{
Sarah M. Mitchell ${ }^{\dagger}$, Yuanhui Xiang ${ }^{\dagger\lrcorner}$, Rachael Matthews ${ }^{\dagger}{ }^{\wedge}$, Alexis M. Amburgey ${ }^{\dagger}$, \\ Emily B. Pentzer $*^{*}$ \\ †Department of Chemistry, Case Western Reserve University, 10900 Euclid Avenue, Cleveland, Ohio 44106, \\ United States \\ ‡Department of Materials Science \& Engineering and Department of Chemistry, Texas A\&M University, 3003 \\ TAMU, College Station, Texas 77843, United States \\ *Corresponding Author (E-mail: emilypentzer@tamu.edu) \\ $\Delta$ Co-second authors \\ Email:smm253@case.edu
}

Table of Contents:

Page

Figure S1 (Control experiment with benzyl alcohol)

Figure S2 (Control experiment with benzyl mercaptan)

Figure S3 (Control experiment with aniline)

Table S1 (Various LAs examined)

Figure S4 (LA Coordination to silyl ketene)

Figure S5 (Space filling models of various silyl ketenes) 

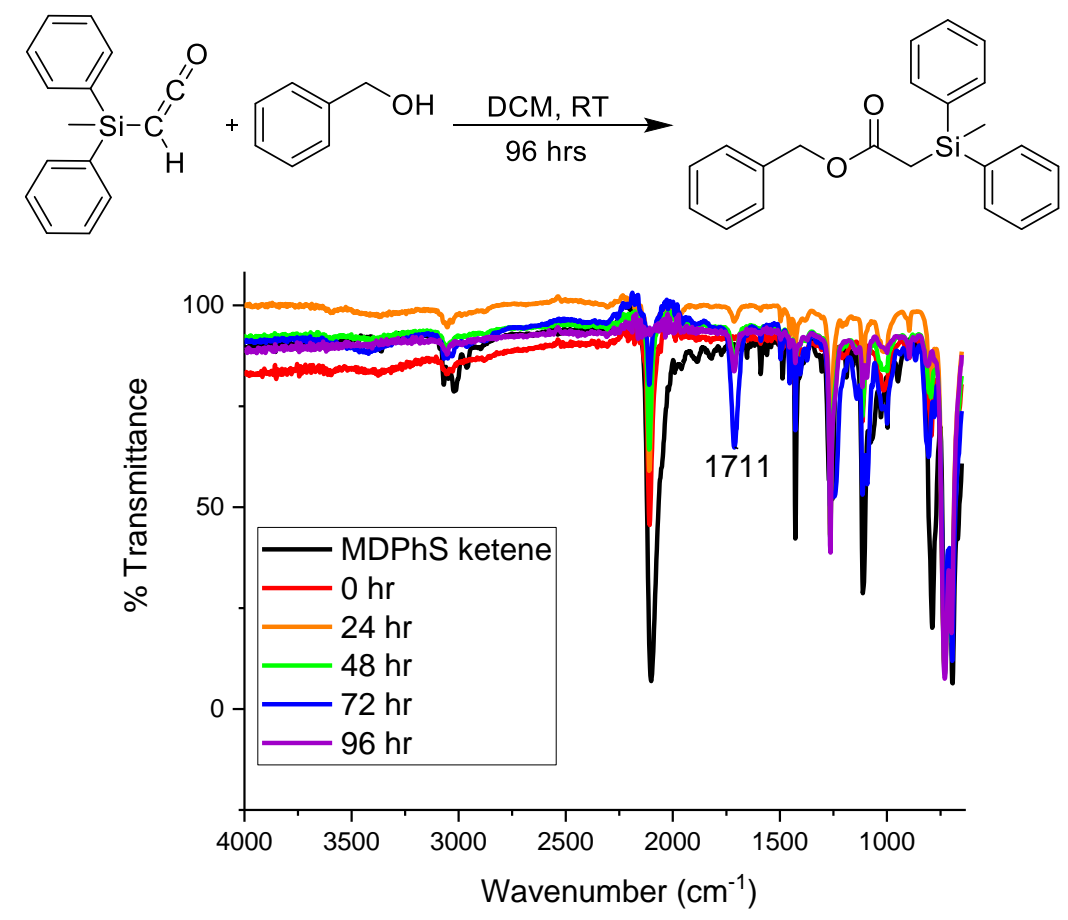

Figure S1. Control experiment performed with no Lewis acid and benzyl alcohol. The reaction was monitored with FTIR to see the consumption of the ketene peak at $\sim 2100 \mathrm{~cm}^{-1}$.

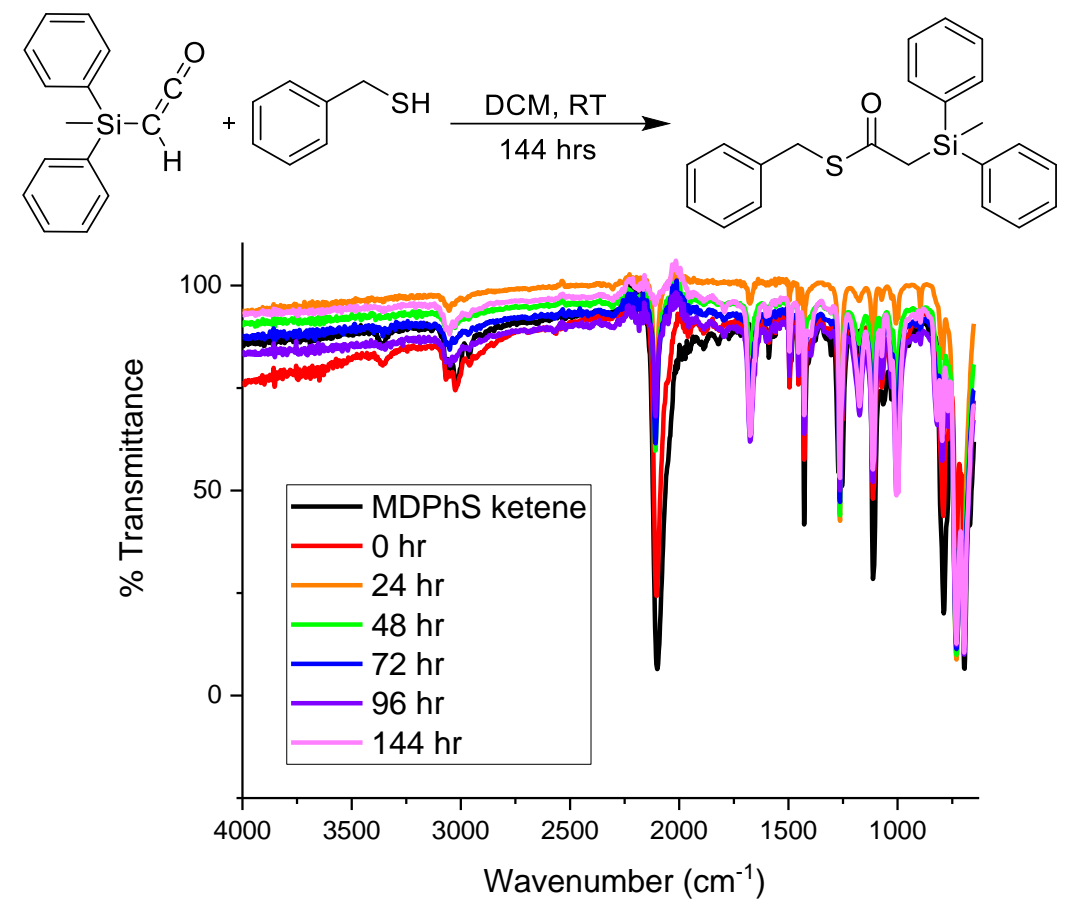

Figure S2. Control experiment performed with no Lewis acid and benzyl mercaptan. The reaction was monitored with FTIR to see the consumption of the ketene peak at $\sim 2100 \mathrm{~cm}^{-1}$. 

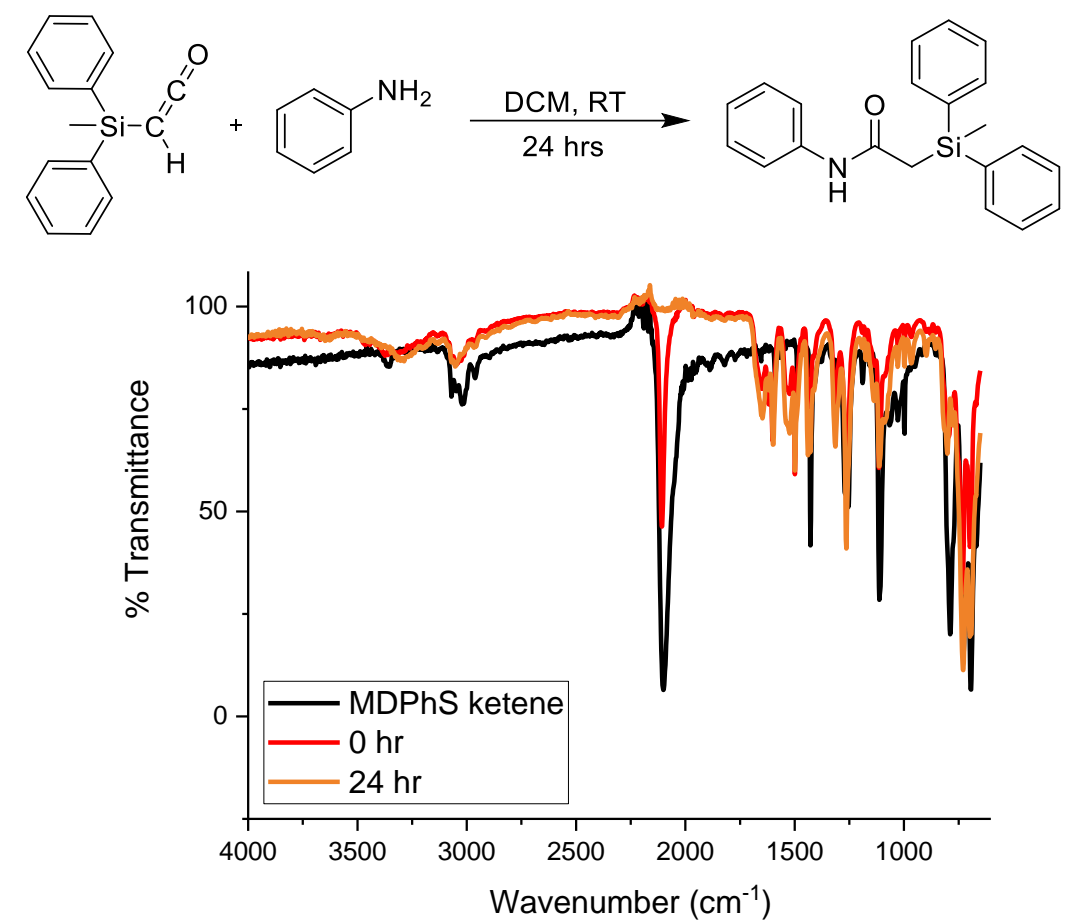

Figure S3. Control experiment performed with no Lewis acid and aniline. The reaction was monitored with FTIR to see the consumption of the ketene peak at $\sim 2100 \mathrm{~cm}^{-1}$.

Table S1. Lewis acid scope.

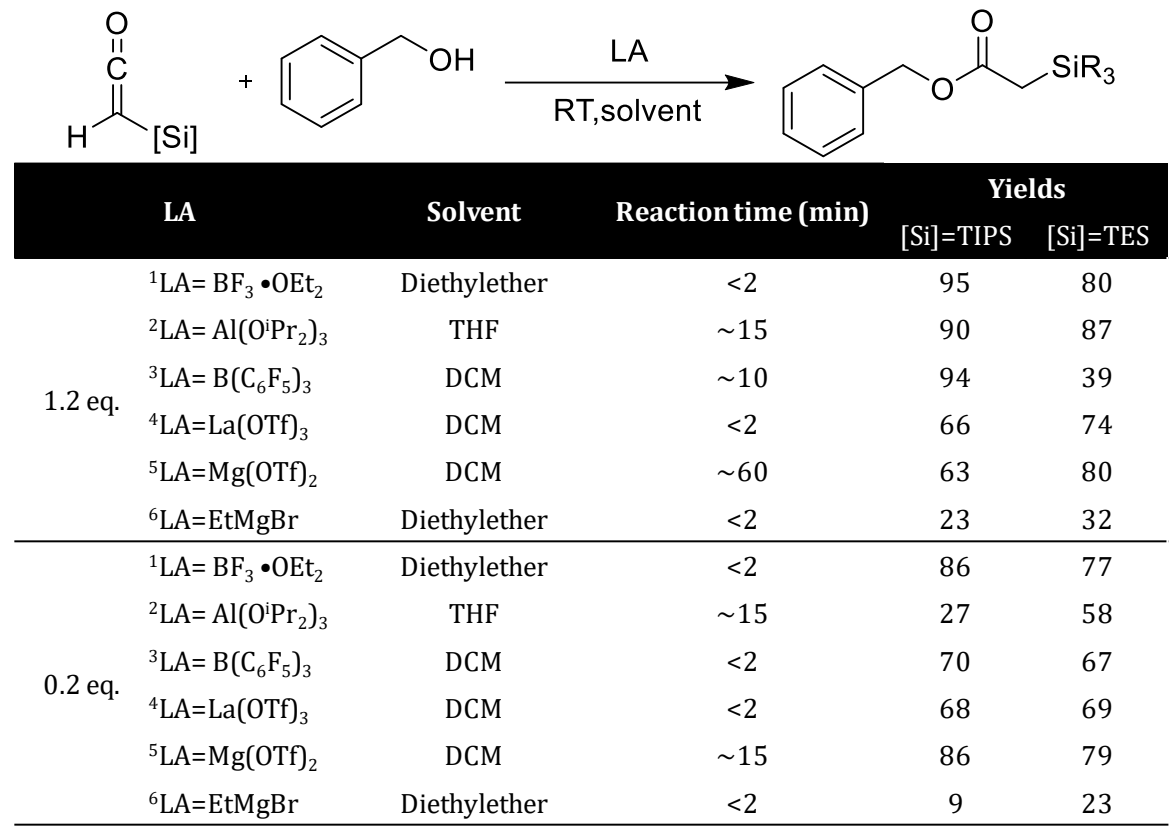

*each LA was added in both 1.2 or 0.2 equivalents to the reaction mixture. It was found that $\mathrm{BF}_{3} \cdot \mathrm{OEt}_{2}$ can also be added in a catalytic amount. 

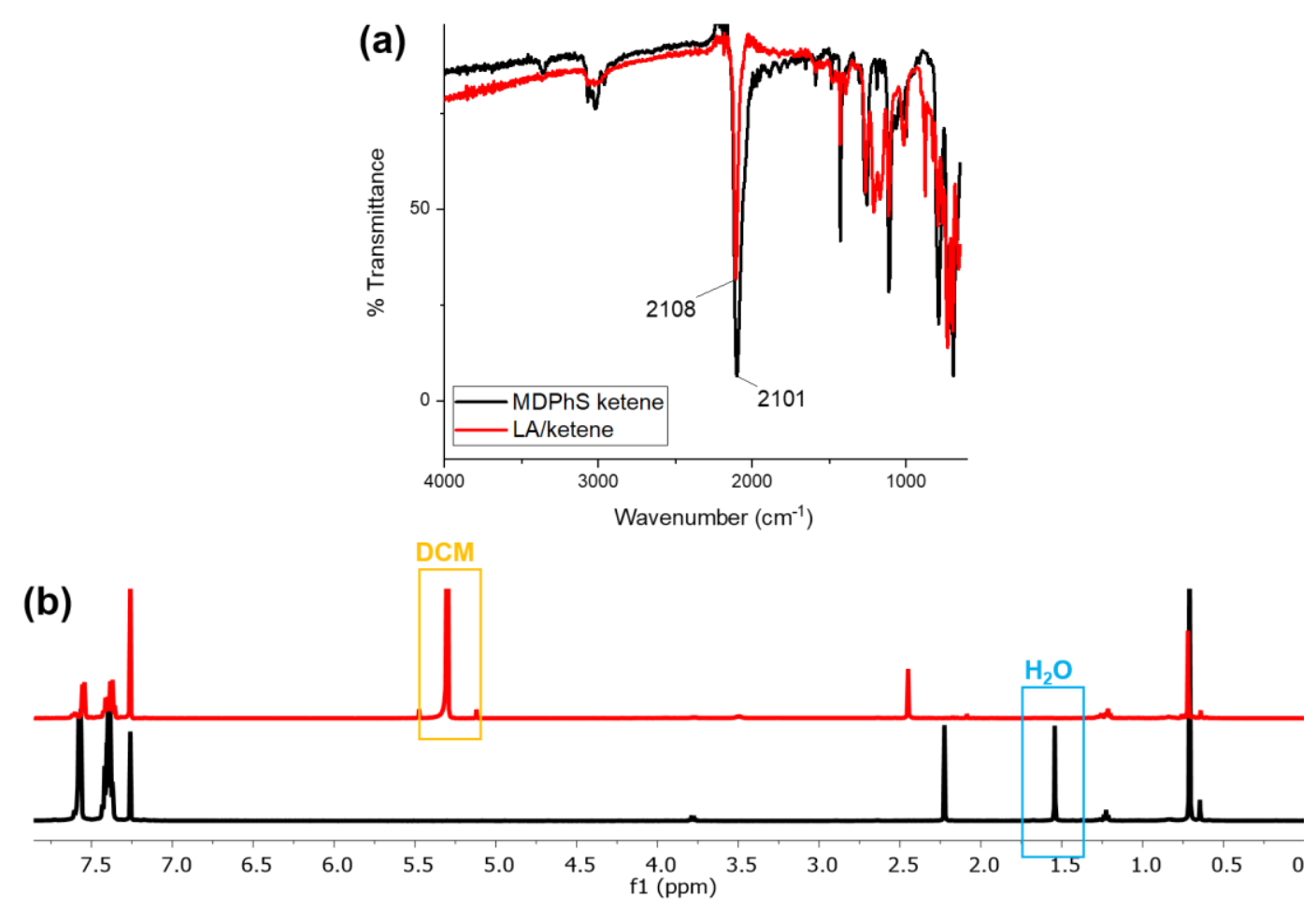

Figure S4. Lewis acid coordination to MDPhS ketene with no presence of nucleophile. (a) FTIR (b) ${ }^{1} \mathrm{H}$ NMR. 
A.<smiles>CC[Si](C=O)(CC)CC</smiles>
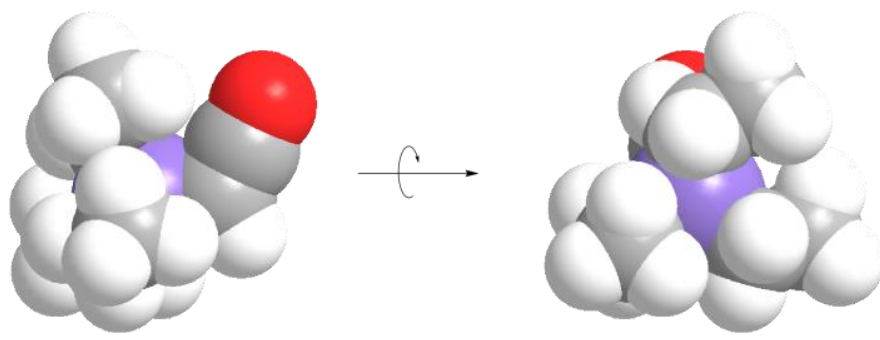

B.<smiles>O=C1OC2CCC1CC21SC2CCC1C2</smiles>

C.
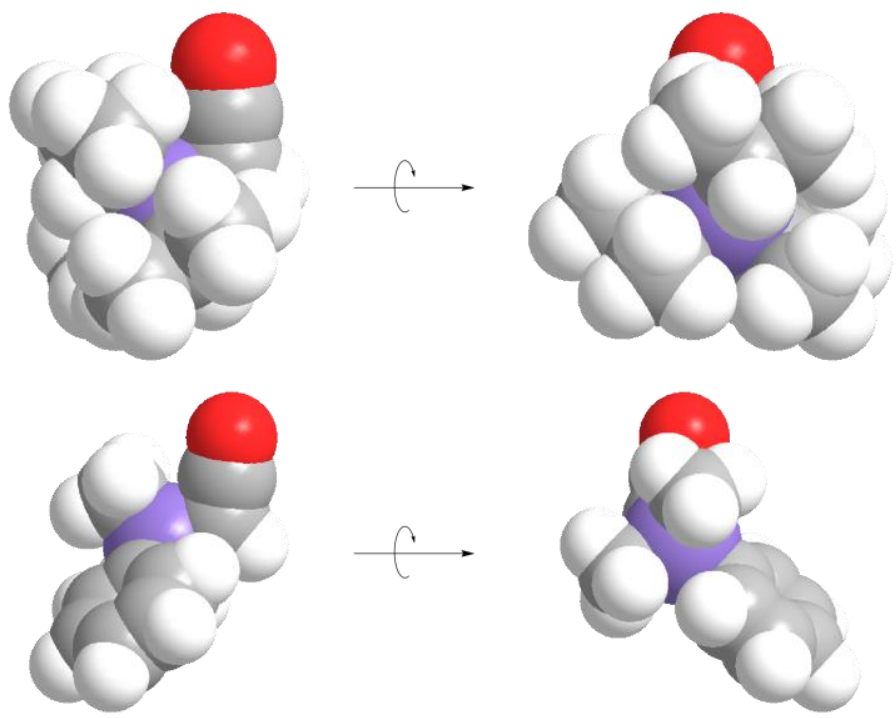

D.<smiles>O=CC1(c2ccccc2)c2ccccc2[SH]1(=O)c1ccccc1</smiles>
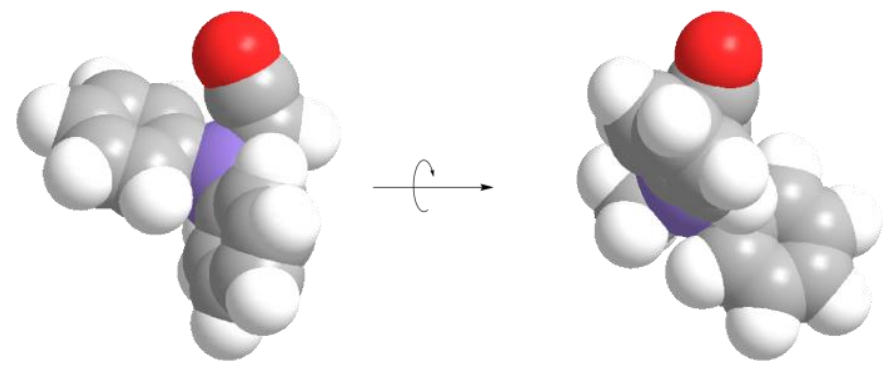

E.<smiles>CC(C)(C)c1ccccc1[SH](=O)(c1ccccc1)c1ccccc1</smiles>
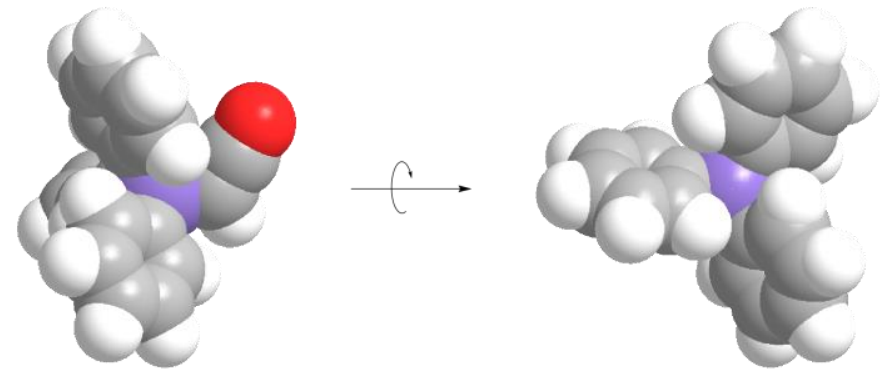

Figure S5. Space filling models of silyl ketenes. (Oxygen-red, silicon-purple) (A) TES ketene (B) TIPS ketene (C) DMPhS ketene (D) MDPhS ketene (E) TPhS ketene. 
Figure S6. Compound 1. (A) FTIR (B) mass spectroscopy<smiles>CCOC(=O)C[Si](C)(c1ccccc1)c1ccccc1</smiles>

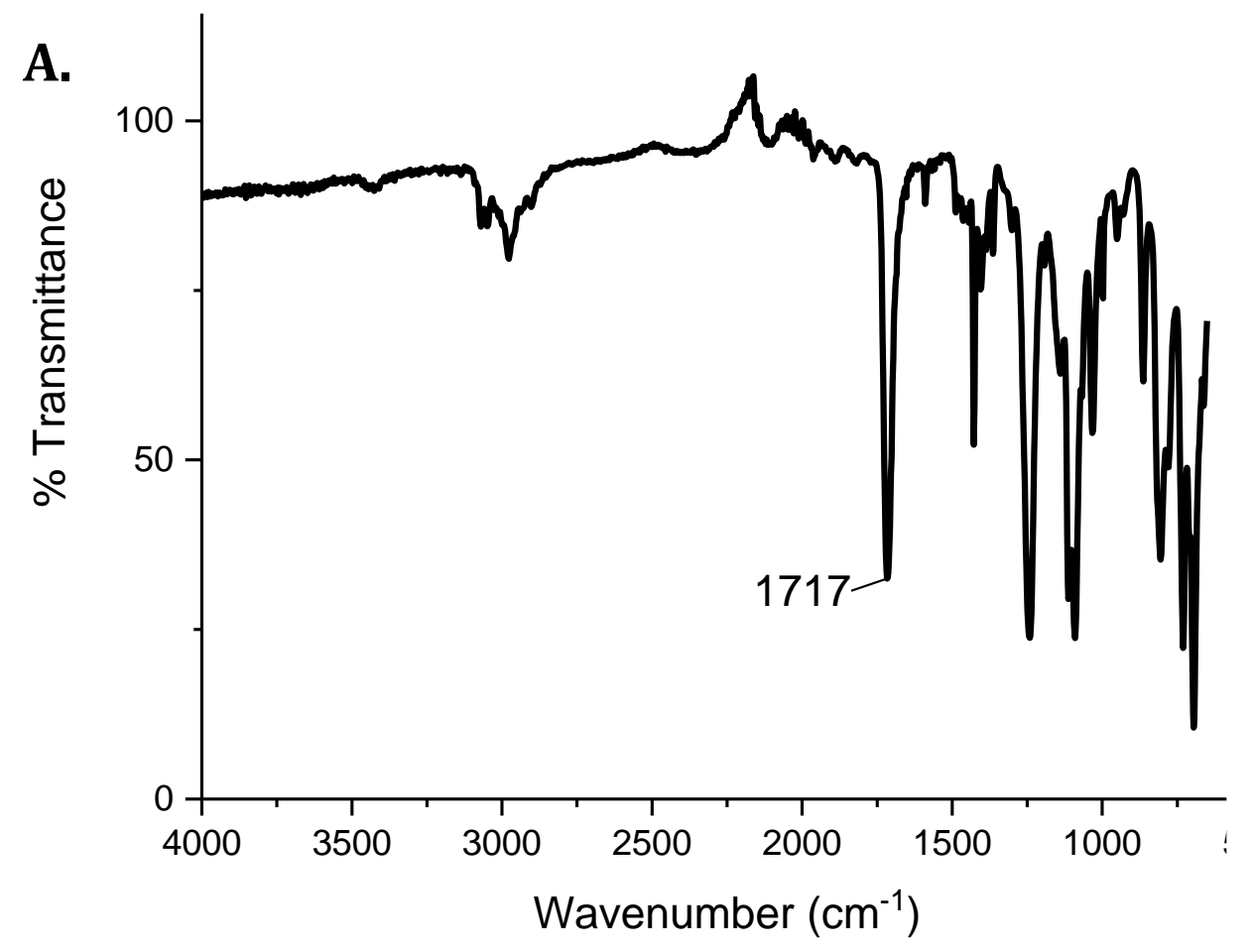


B. 09191907 \#101-108 RT: $0.45-0.48$ AV: 8 SB: 23 0.27-0.37 NL: 7.45E8
T: FTMS + p ESI Full ms [50.0000-500.0000]

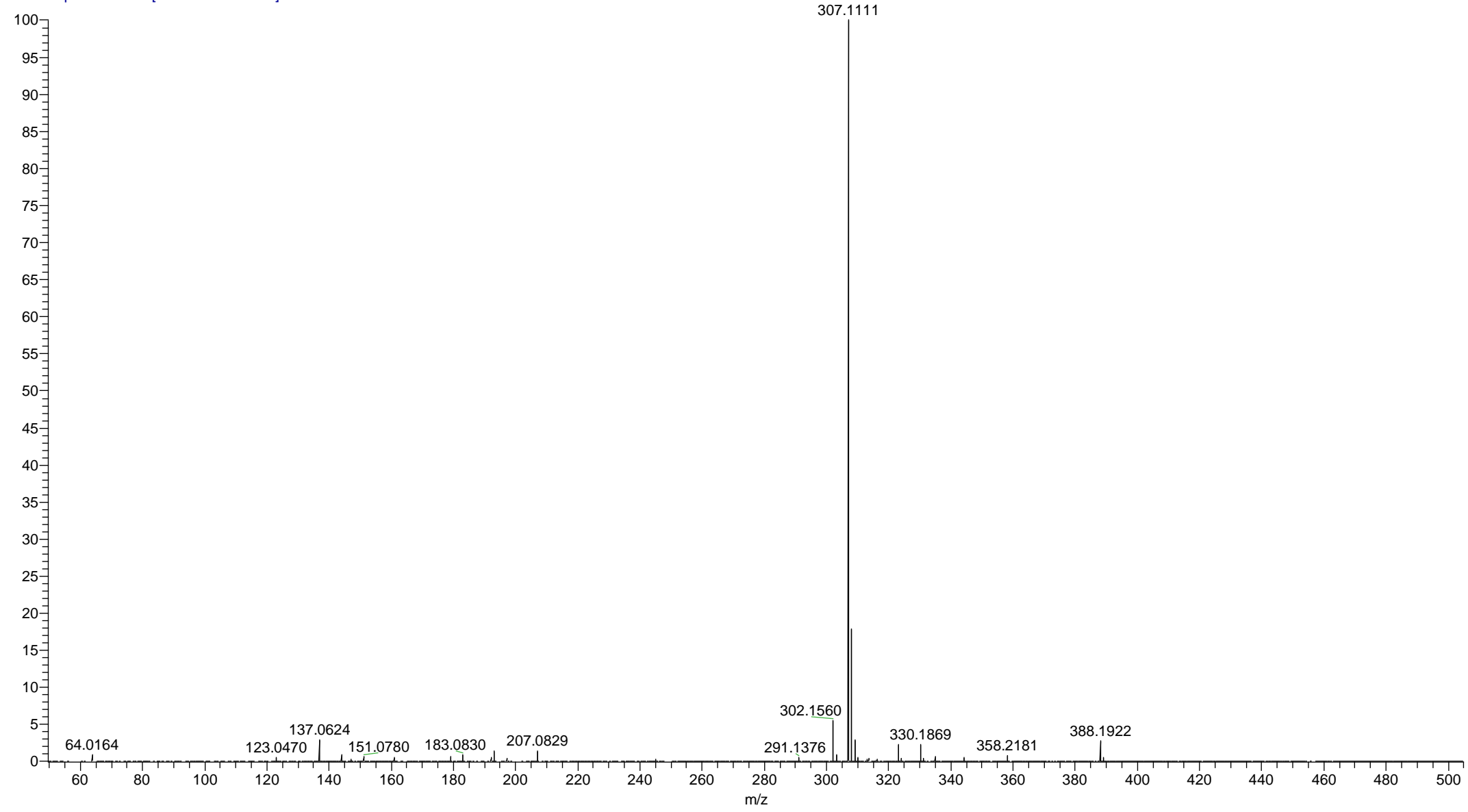


Figure S7. Compound 2. (A) FTIR (B) ${ }^{1} \mathrm{H}$ NMR (C) ${ }^{13} \mathrm{C}$ NMR (D) ${ }^{29}$ Si NMR (E) mass spectroscopy<smiles>CC(C)OC(=O)C[Si](C)(c1ccccc1)c1ccccc1</smiles>

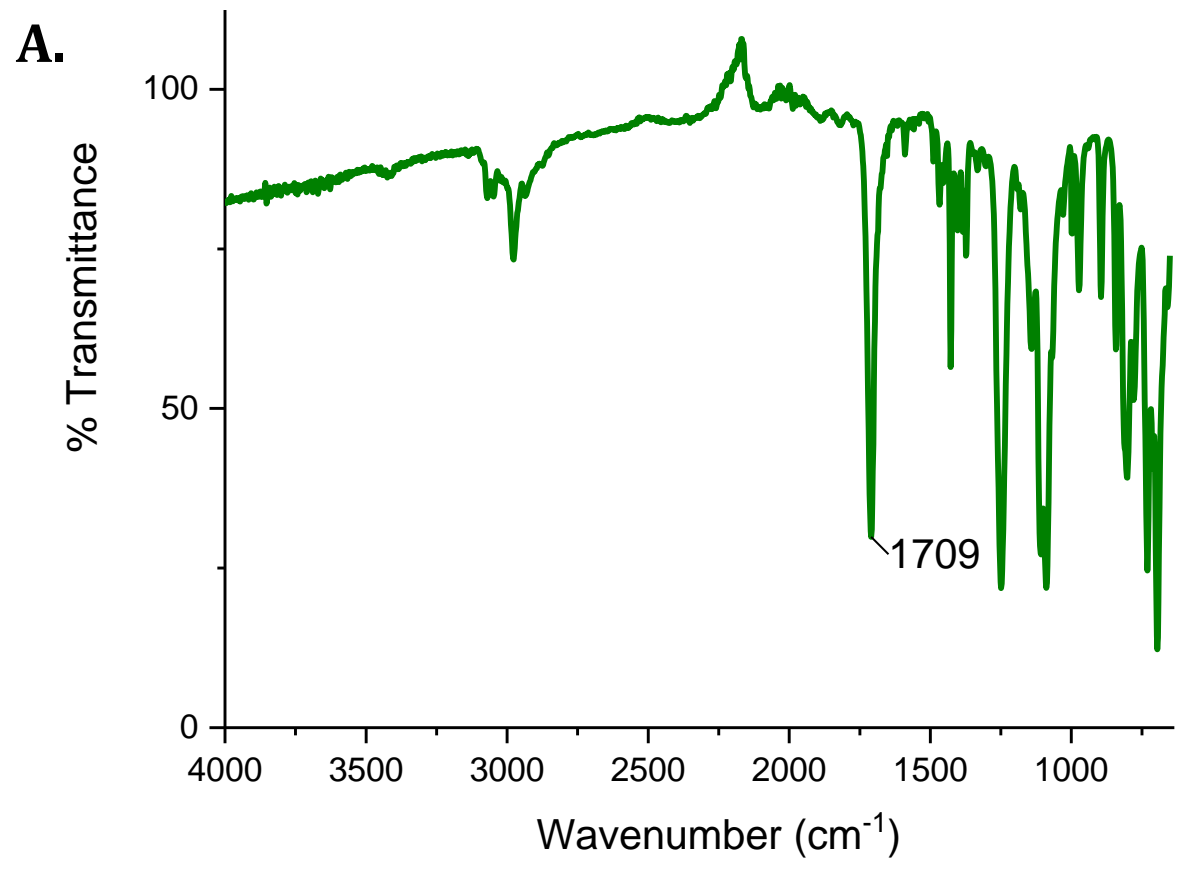


B.
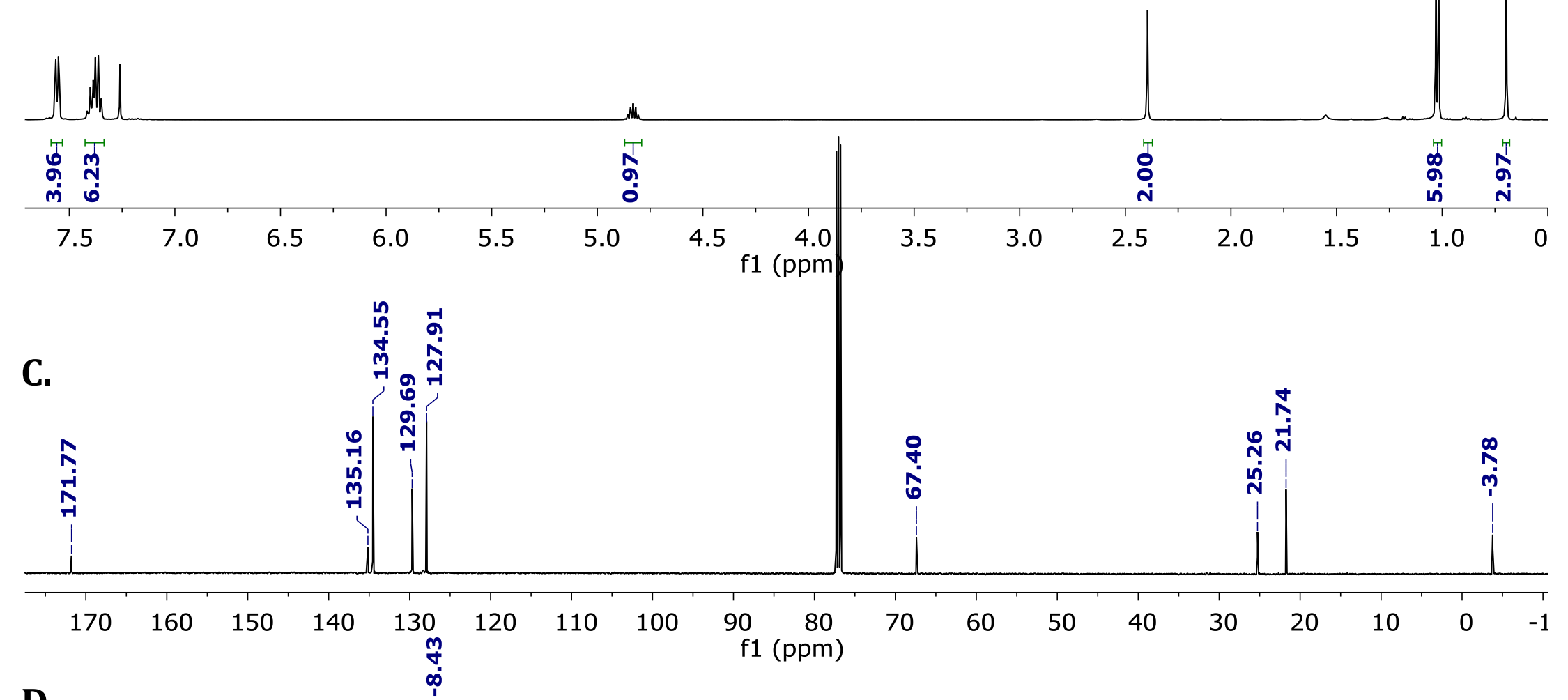

D.

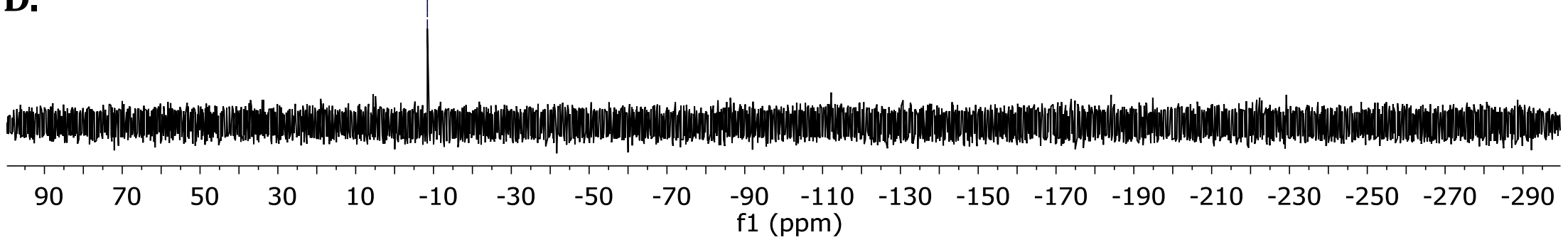


E.

09191908 \#116-133 RT: 0.52-0.59 AV: 18 SB: 22 0.32-0.41 NL: 1.26E8 T: FTMS + p ESI Full ms [50.0000-500.0000]

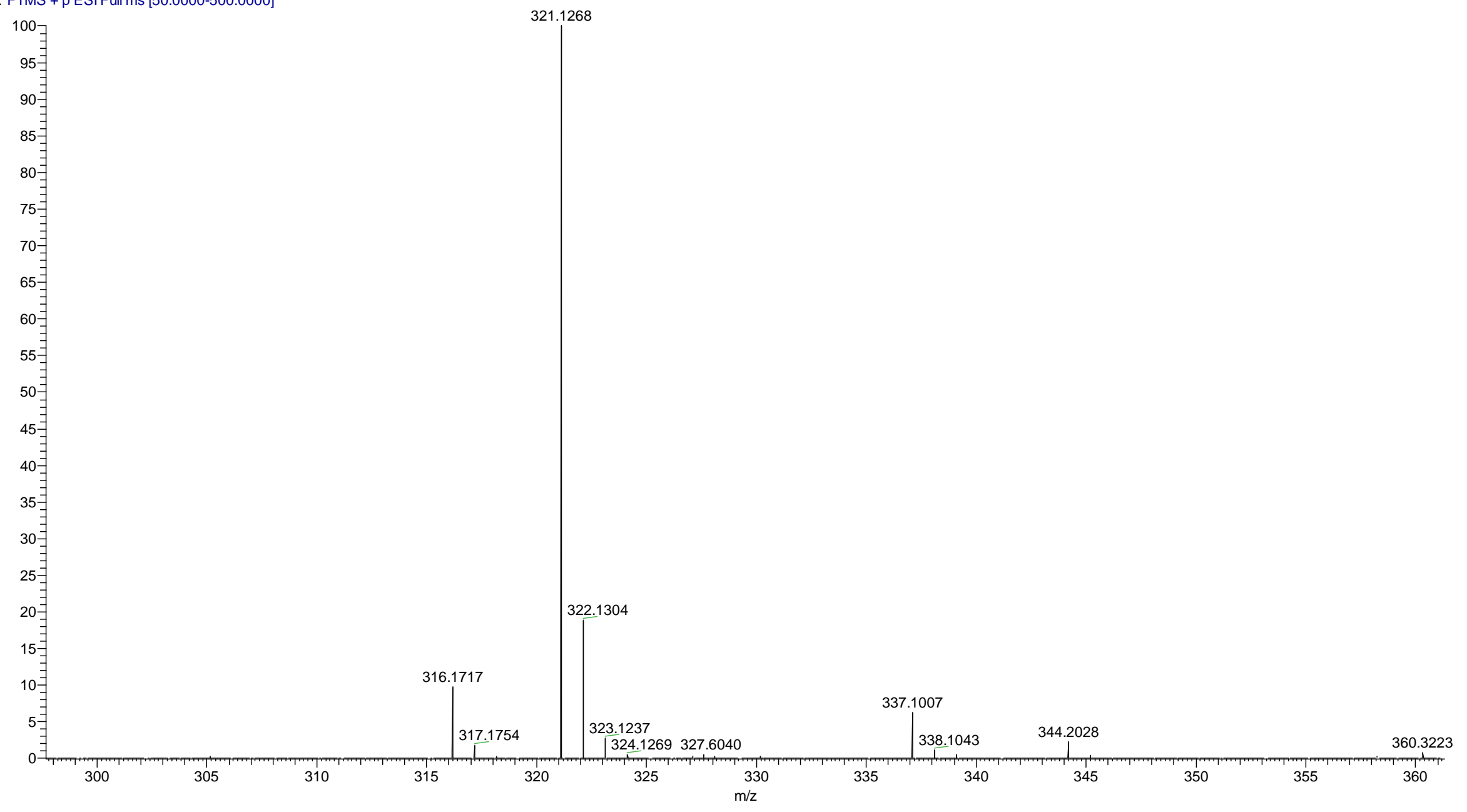


Figure S8. Compound 3. (A) FTIR (B) ${ }^{1} \mathrm{H}$ NMR (C) ${ }^{13} \mathrm{C}$ NMR (D) ${ }^{29}$ Si NMR (E) mass spectroscopy<smiles>CC(C)(C)OC(=O)C[Si](C)(c1ccccc1)c1ccccc1</smiles>

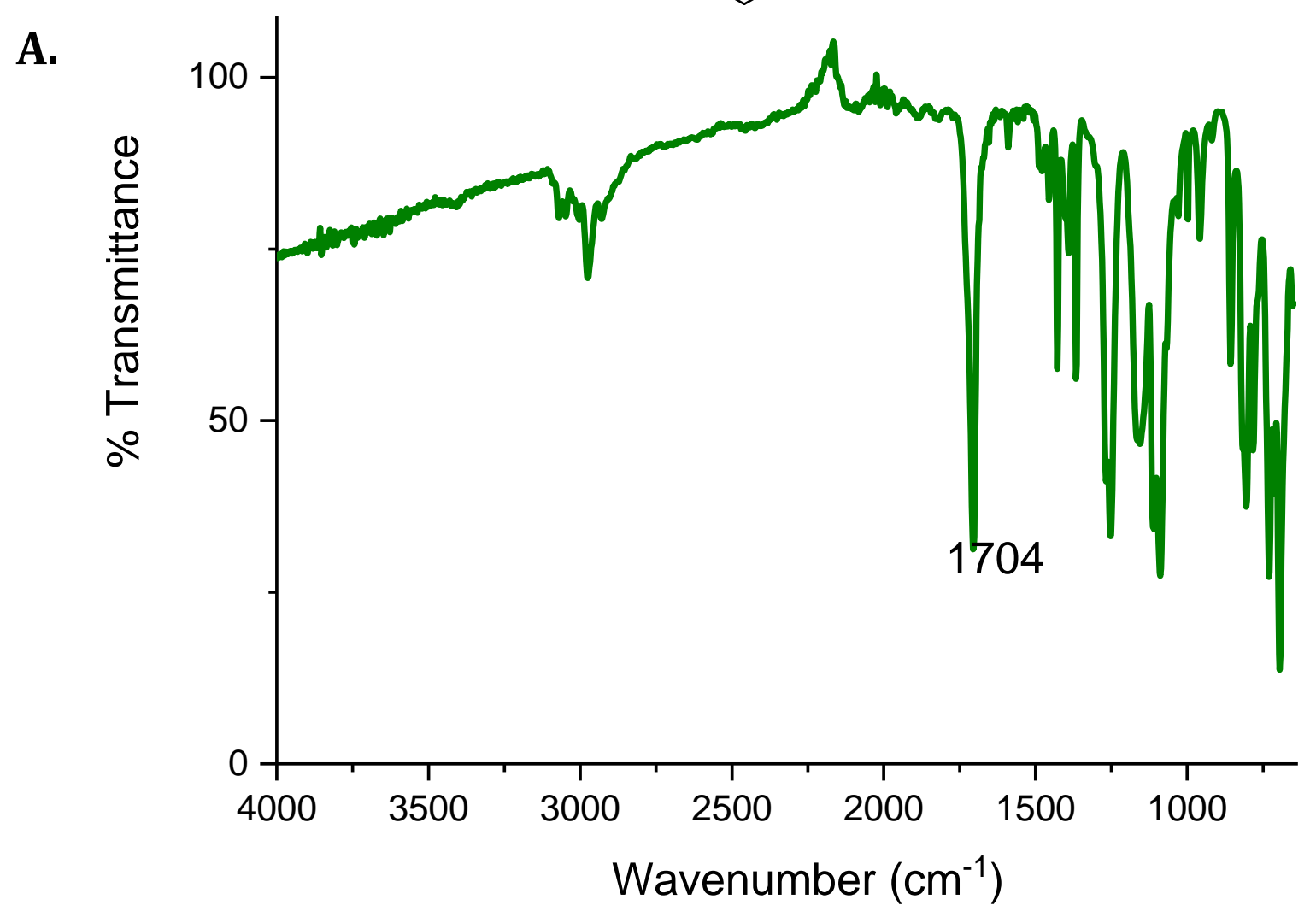




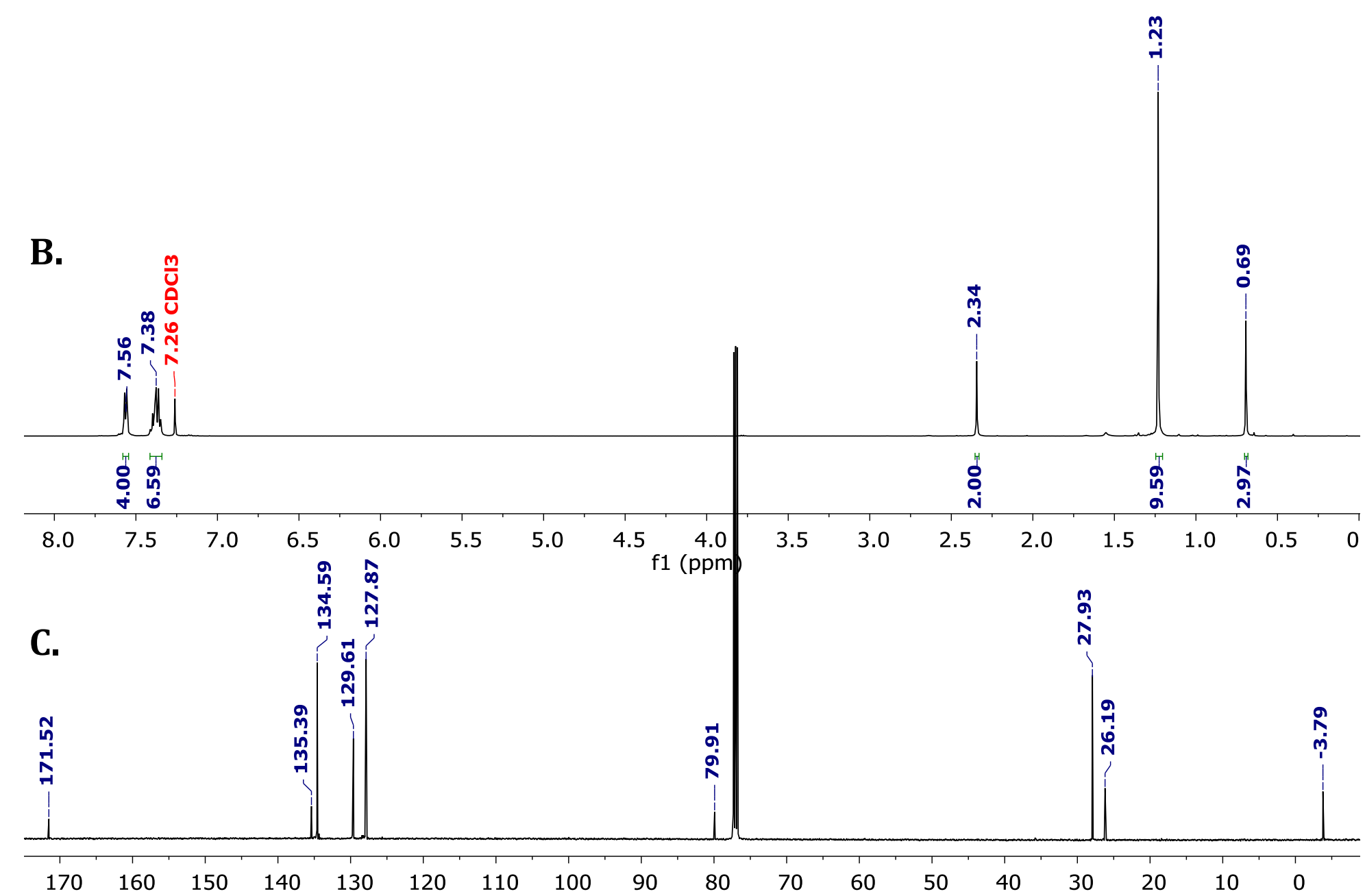

D.

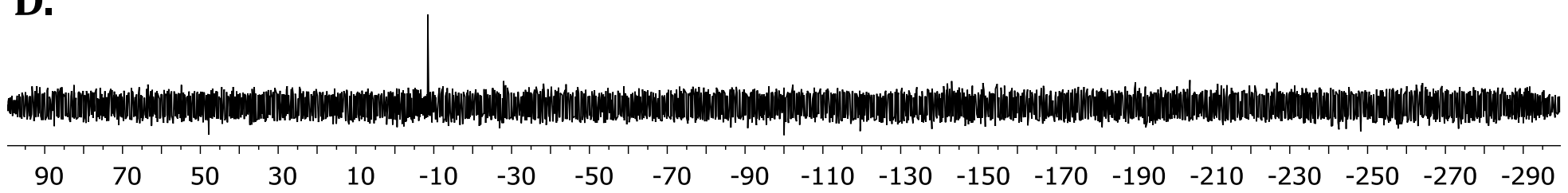




\section{E.}

09191909a \#109-118 RT: 0.49-0.53 AV: 10 SB: 23 0.28-0.38 NL: 1.52E8

T: FTMS + p ESI Full ms [50.0000-500.0000]

100

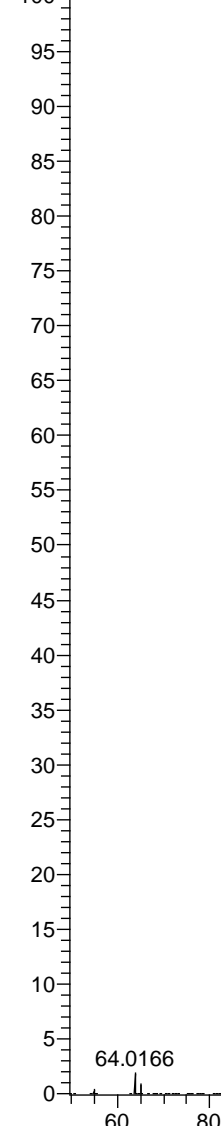

$$
\text { SB: } 23 \text { 0.28-0.38 NL: } 1.52 E 8
$$

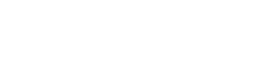


Figure S9. Compound 4 (A) FTIR (B) ${ }^{1} \mathrm{H}$ NMR (C) ${ }^{13} \mathrm{C}$ NMR (D) ${ }^{29}$ Si NMR (E) mass spectroscopy<smiles>C[Si](CC(=O)Oc1ccccc1)(c1ccccc1)c1ccccc1</smiles>

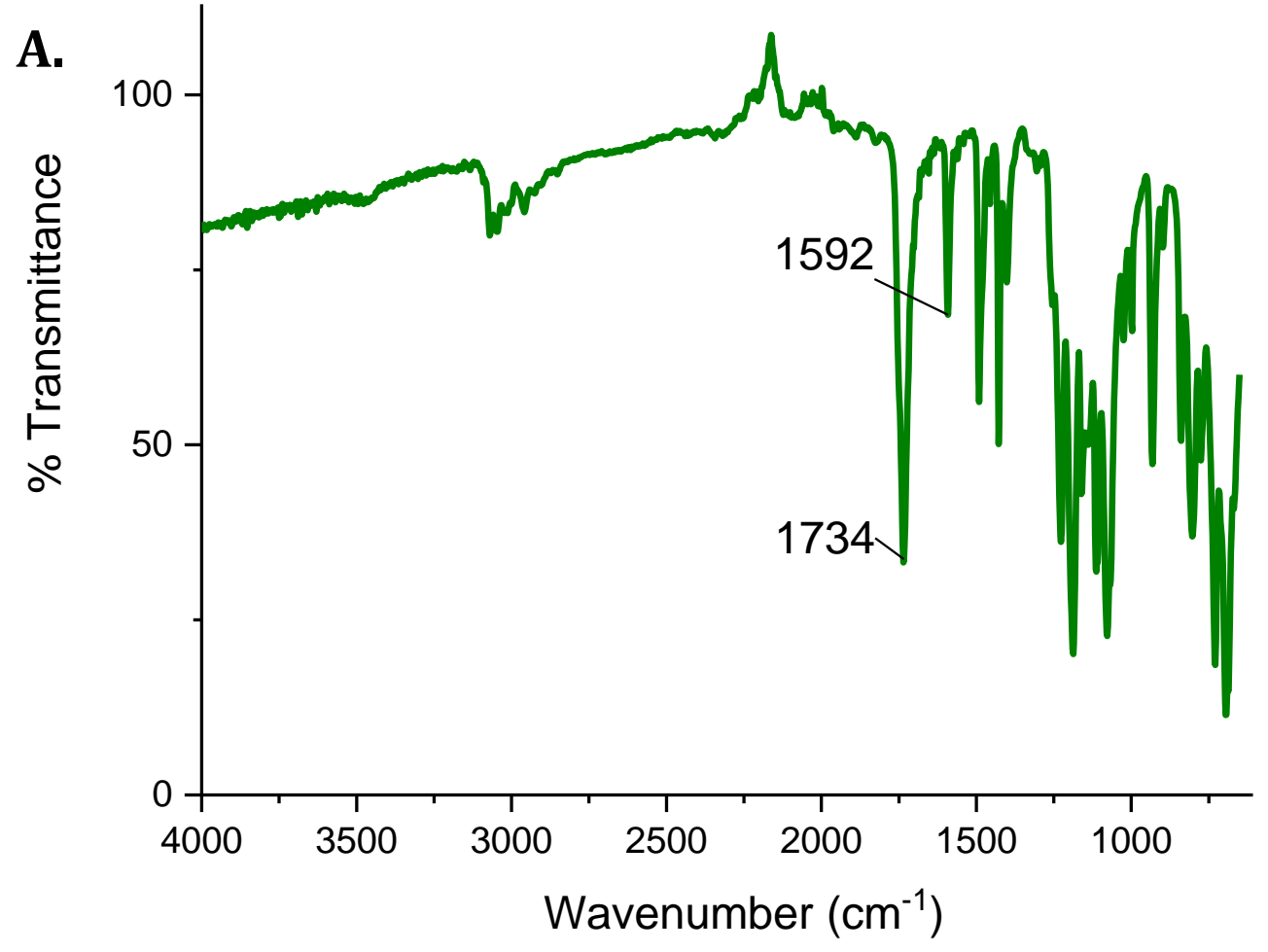




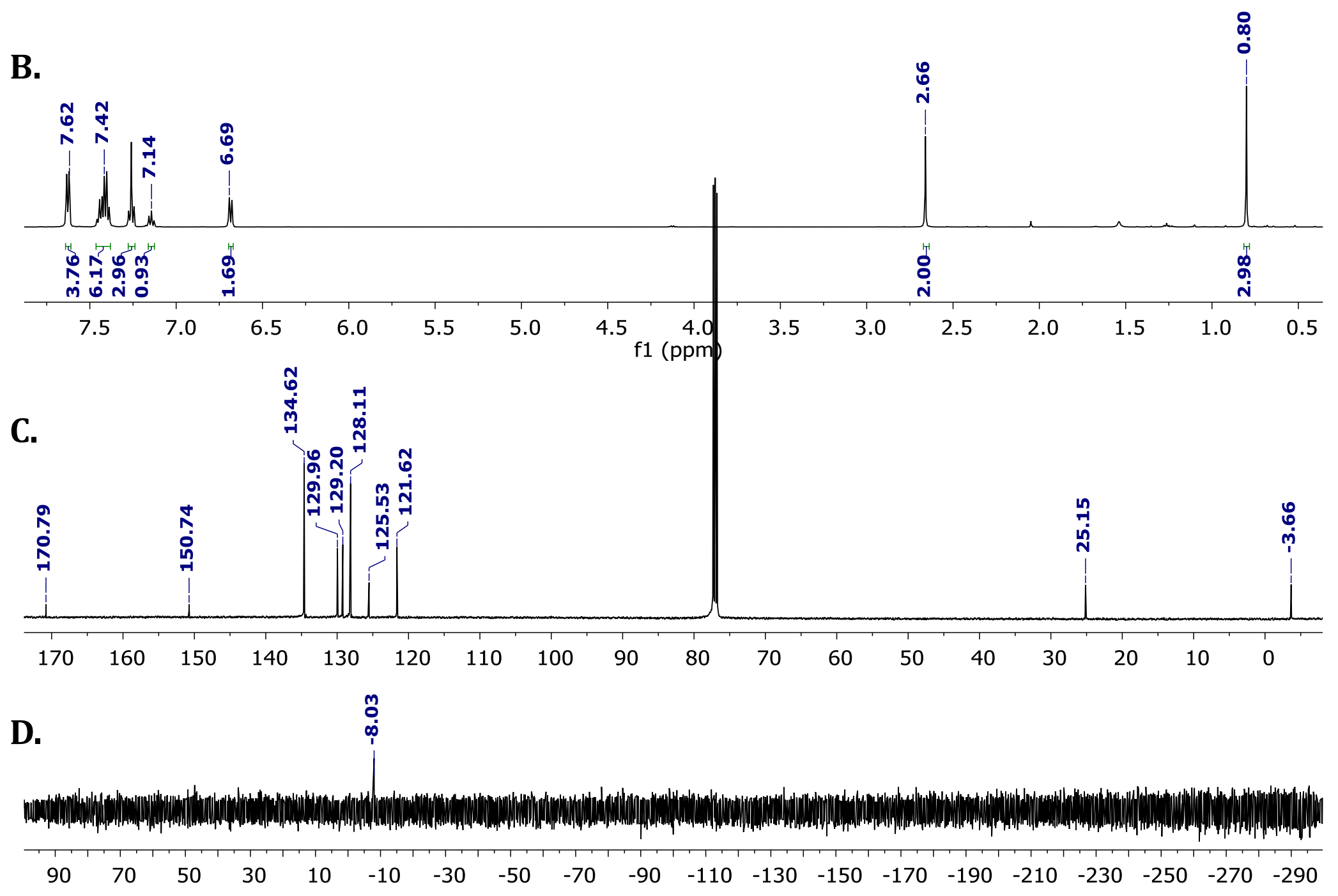


E.

09191910\#148-167 RT: 0.66-0.74 AV: 20 SB: 27 0.37-0.49 NL: 8.91E7 T: FTMS + p ESIFull ms [50.0000-500.0000]

$95 \frac{\text { G }}{9}$

90 手

$85-$

$80-$

75 氺

$70 \exists$

65 =

60 ㄱ.

$55^{-}$

$50=$

$45^{-}$

${ }^{40}-$

30 긍

$25 \exists$

20 큭

15 年

10

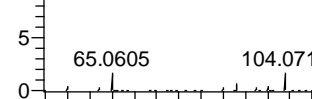

355.1112

$378.1871 \quad 436.1926$ 406.2185 $480+500$ 
Figure S10. Compound 5 (A) FTIR (B) ${ }^{1} \mathrm{H}$ NMR (C) ${ }^{13} \mathrm{C}$ NMR (D) ${ }^{29}$ Si NMR (E) mass spectroscopy<smiles>COc1ccc(OC(=O)C[Si](C)(c2ccccc2)c2ccccc2)cc1</smiles>

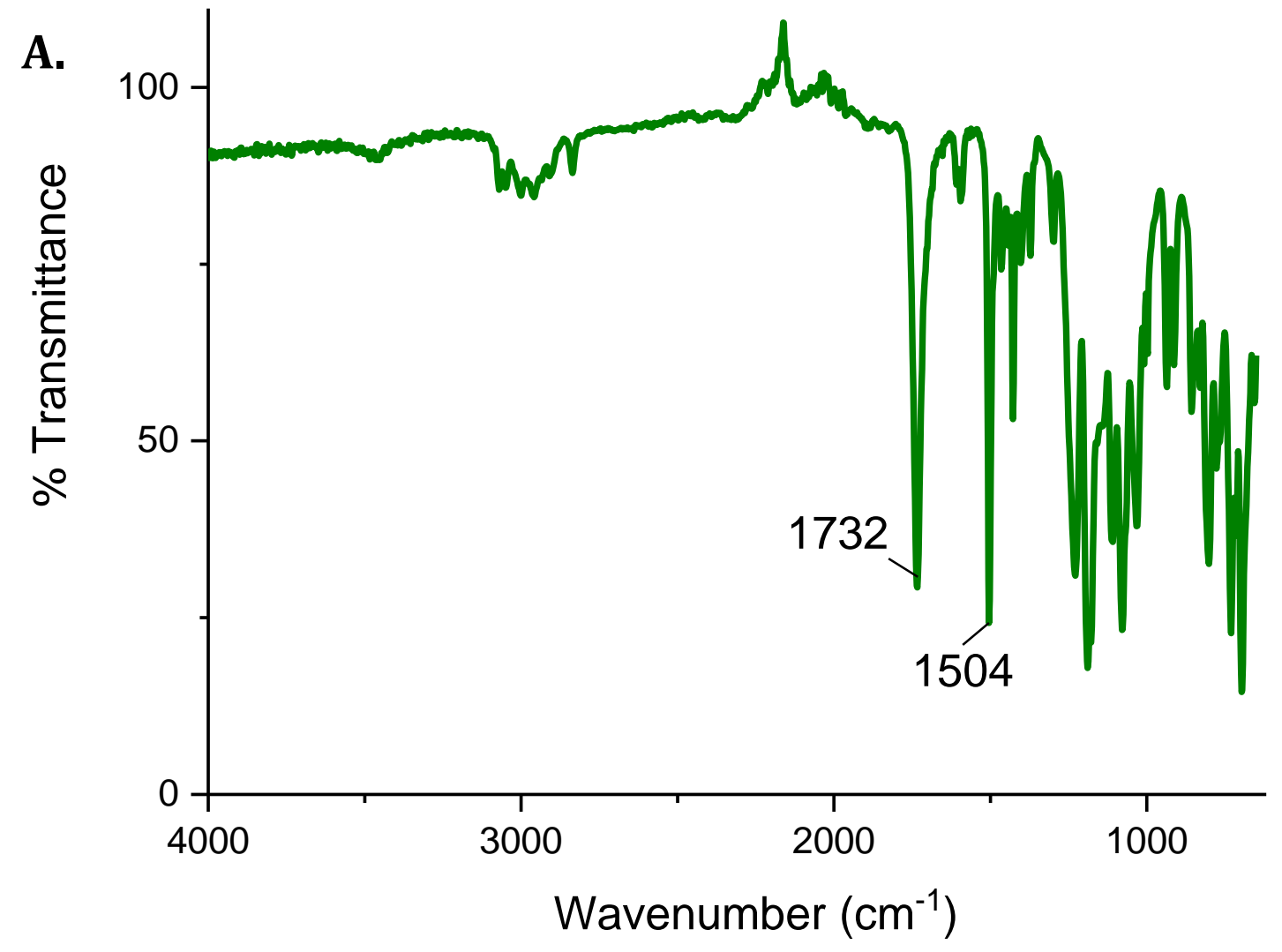



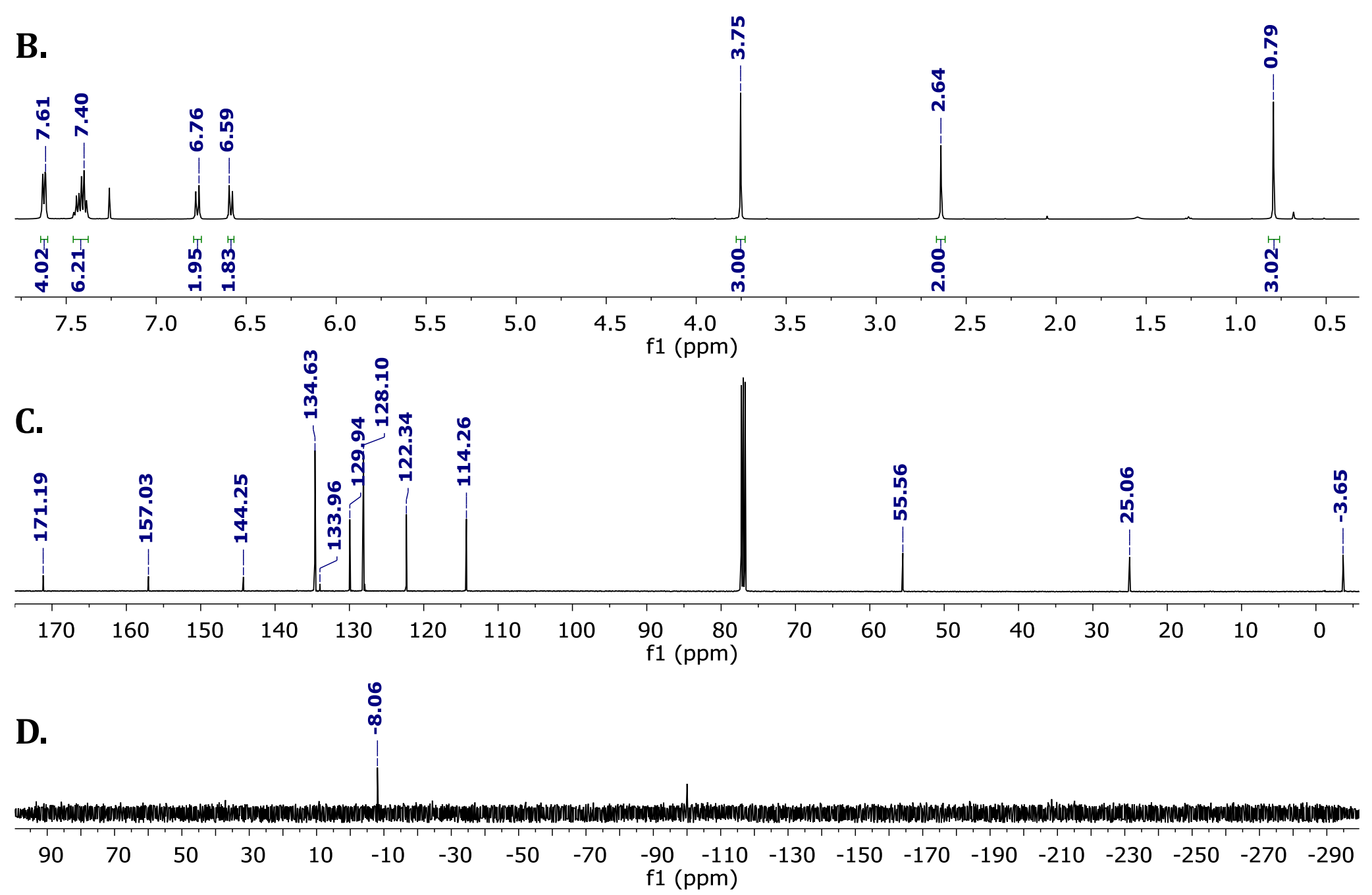
compound 5

E.

09191911 \#111-114 RT: 0.49-0.51 AV: 4 SB: 28 0.23-0.35 NL: 5.32E7 T: FTMS + p ESI Full ms [50.0000-500.0000]

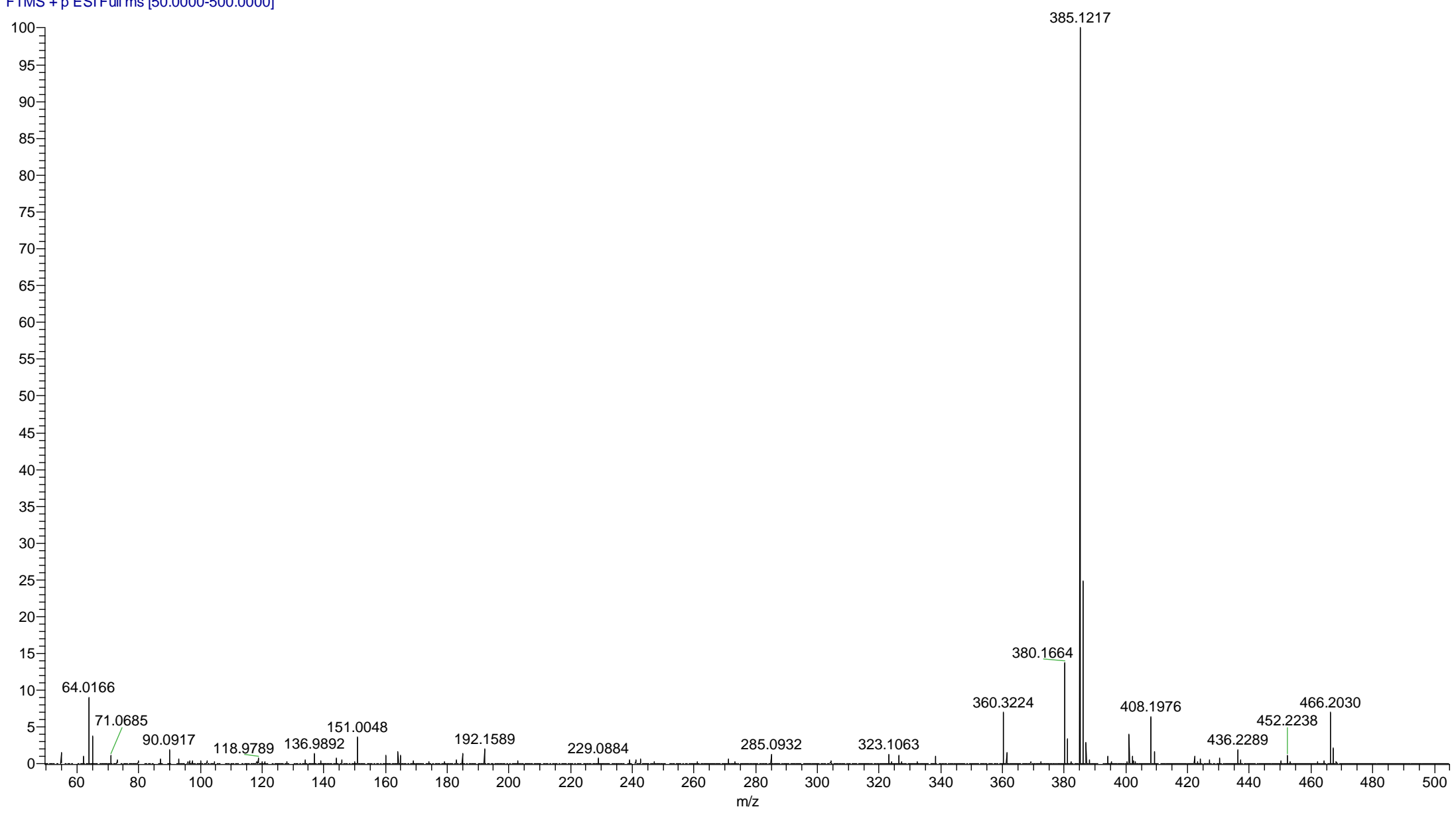


Figure S11. Compound 6 (A) FTIR (B) ${ }^{1} \mathrm{H}$ NMR (C) ${ }^{13} \mathrm{C}$ NMR (D) ${ }^{29} \mathrm{Si}$ NMR (E) mass spectroscopy<smiles>COc1cccc(OC(=O)C[Si](C)(c2ccccc2)c2ccccc2)c1</smiles>

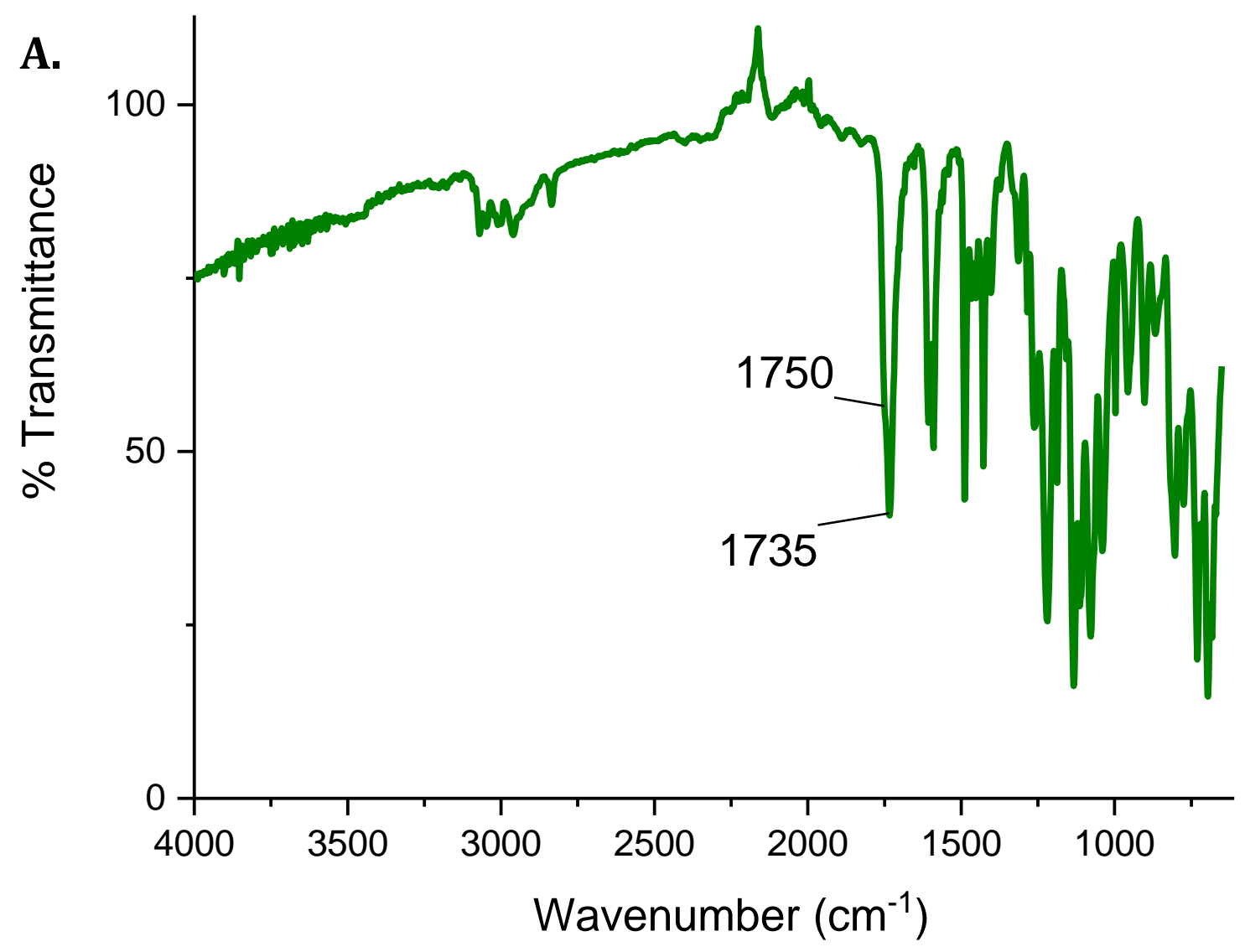


B.
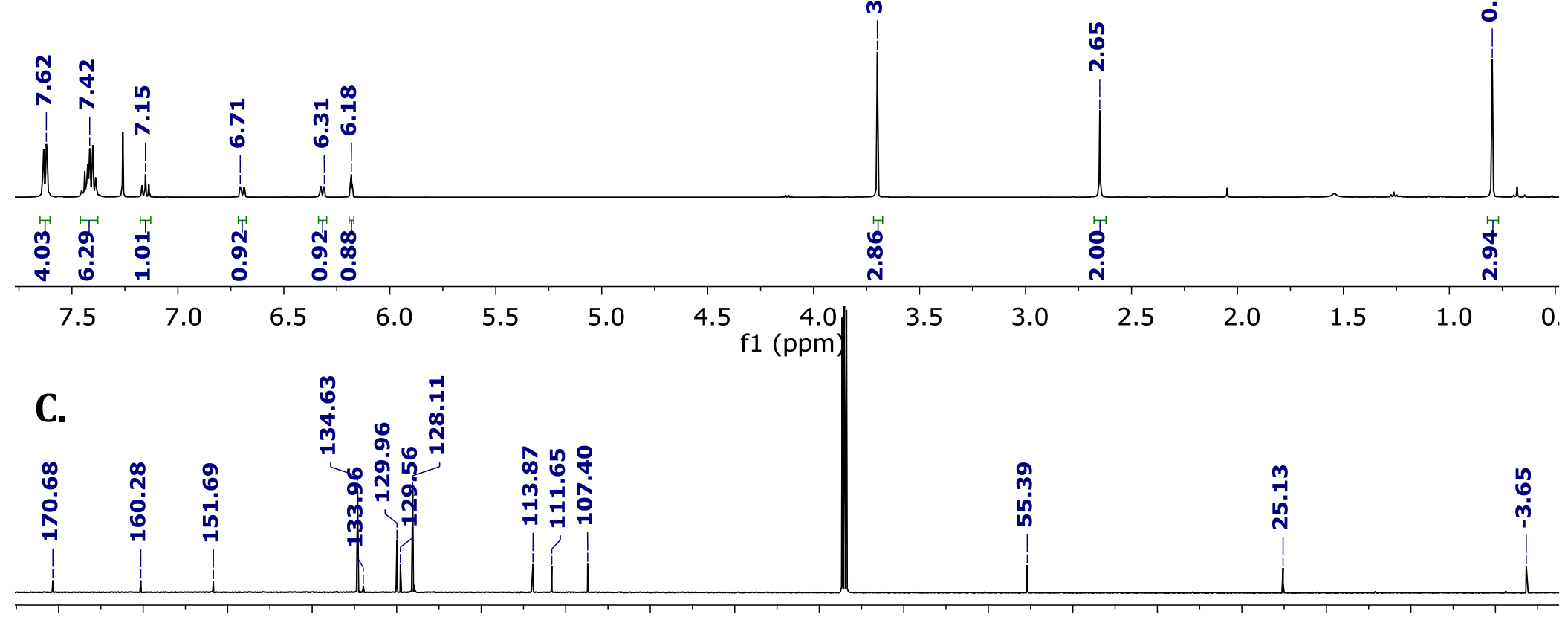

$\begin{array}{lllllllll}170 & 160 & 150 & 140 & 130 & 120 & 110 & 100 & 90 \begin{array}{r}80 \\ \mathrm{f} 1(\mathrm{ppm})\end{array}\end{array}$

D.

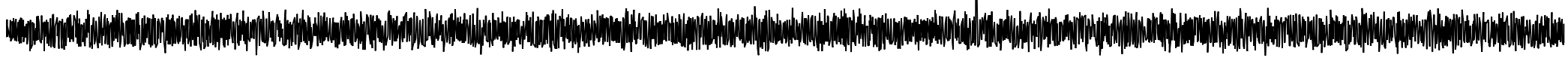

60

$50 \quad 40$

$\begin{array}{lll}30 & 20 & 10\end{array}$

$\begin{array}{lllllll}-10 & -20 & -30 & -40 & -50 & -60 & -7\end{array}$ 
E.

09191912 \#103-114 RT: 0.46-0.51 AV: 12 SB: 20 0.33-0.41 NL: 4.40E7 T: FTMS + p ESI Full ms [50.0000-500.0000]

100

$95=$

$90=$

85

$80=$

$75^{-}$

70

65

60

$50=$

45

40

$35=$

$30=$

$25=$

20 再 64.0165

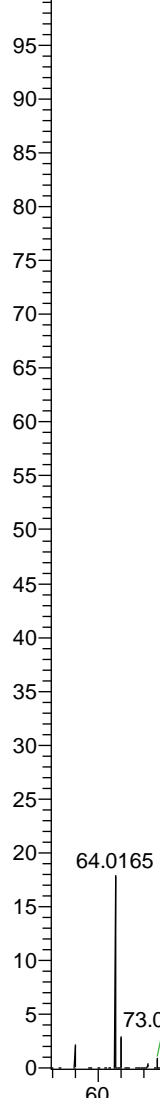

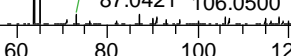

139.0498

140 compound 6

185.1163

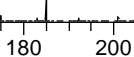

385.1217

380.1664

$408.1976 \quad 466.2030$

| 436.2287

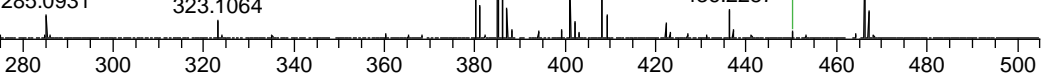


Figure S12. Compound 7 (A) FTIR (B) ${ }^{1} \mathrm{H}$ NMR (C) ${ }^{13} \mathrm{C}$ NMR (D) ${ }^{29}$ Si NMR (E) mass spectroscopy<smiles>COc1ccccc1OC(=O)C[Si](C)(c1ccccc1)c1ccccc1</smiles>

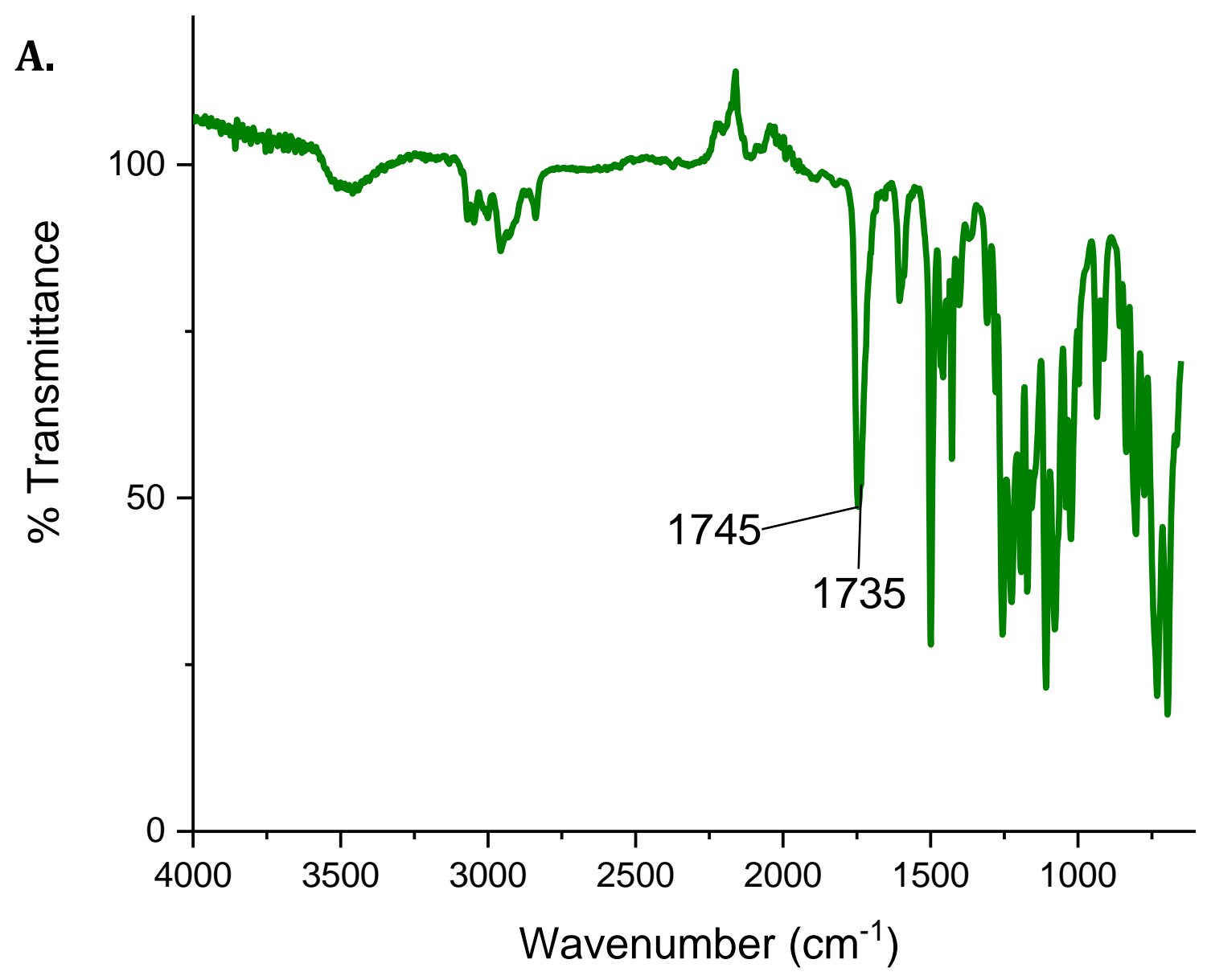




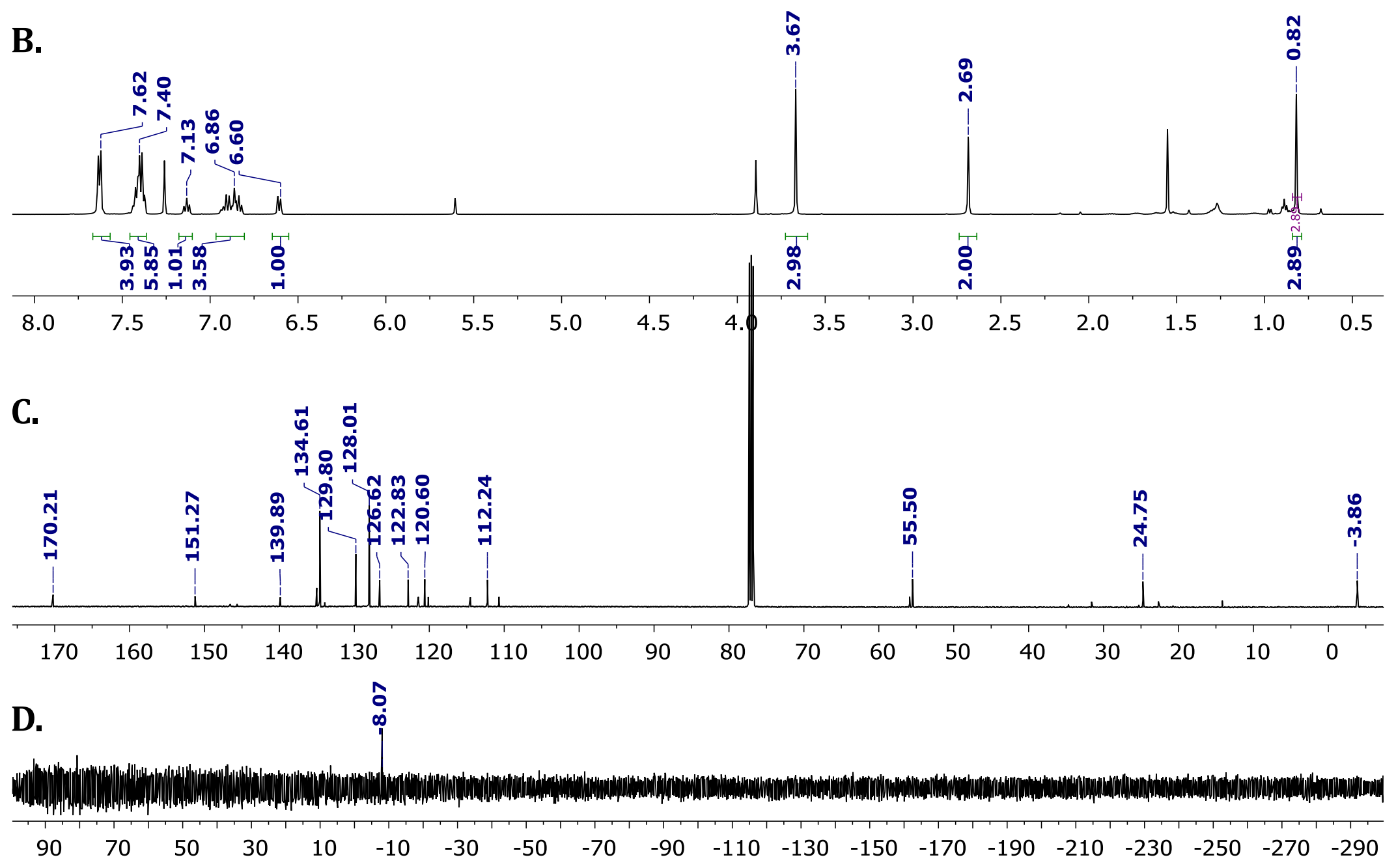


E.

10101909a \#104-129 RT: 0.46-0.57 AV: 26 SB: 23 0.24-0.33 NL: 5.01E6 T: FTMS + p ESI Full ms [50.0000-500.0000]

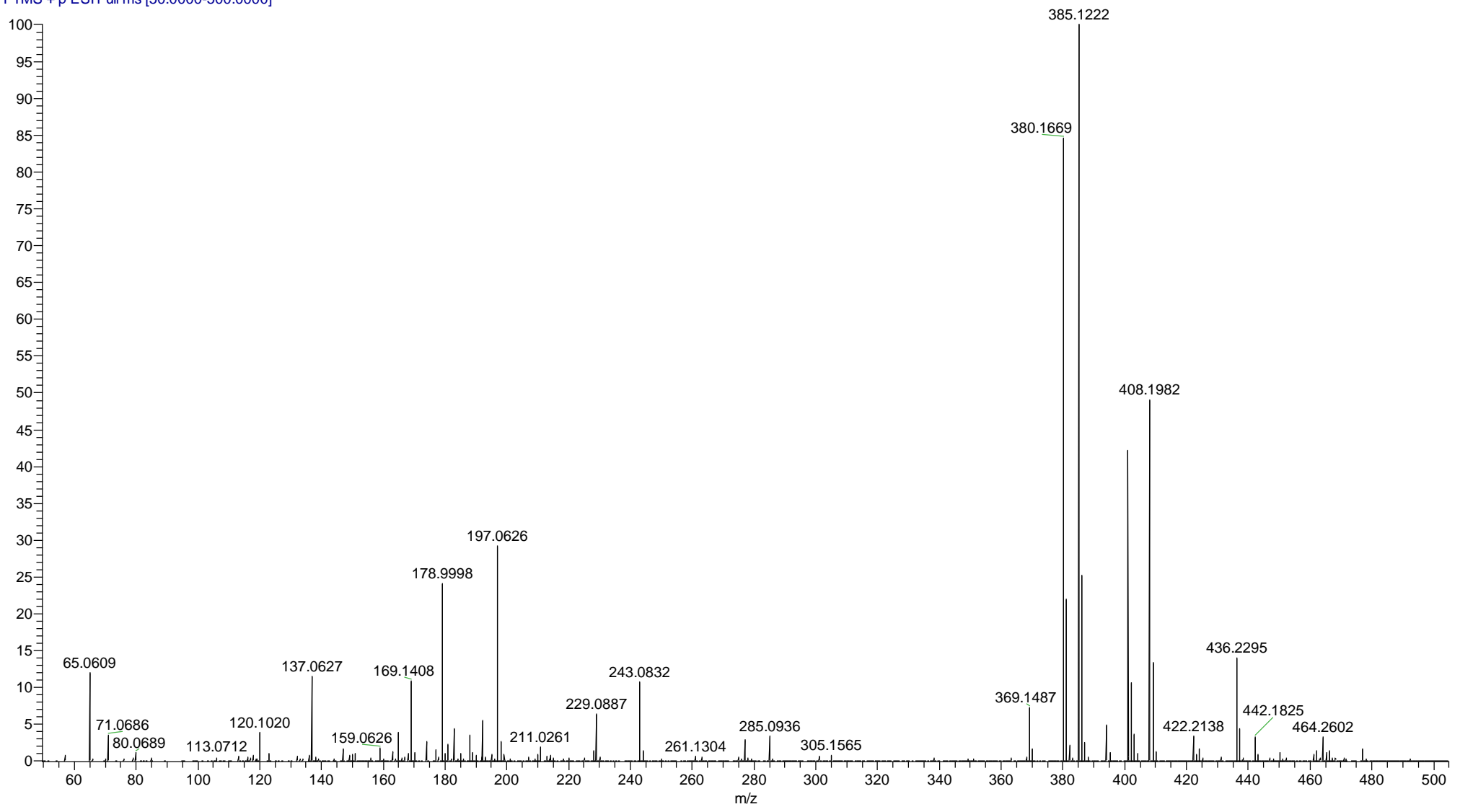


Figure S13. Compound 8 (A) FTIR (B) ${ }^{1} \mathrm{H}$ NMR (C) ${ }^{13} \mathrm{C}$ NMR (D) ${ }^{29}$ Si NMR (E) mass spectroscopy<smiles>CC(=O)N[C@@H](C)O</smiles>

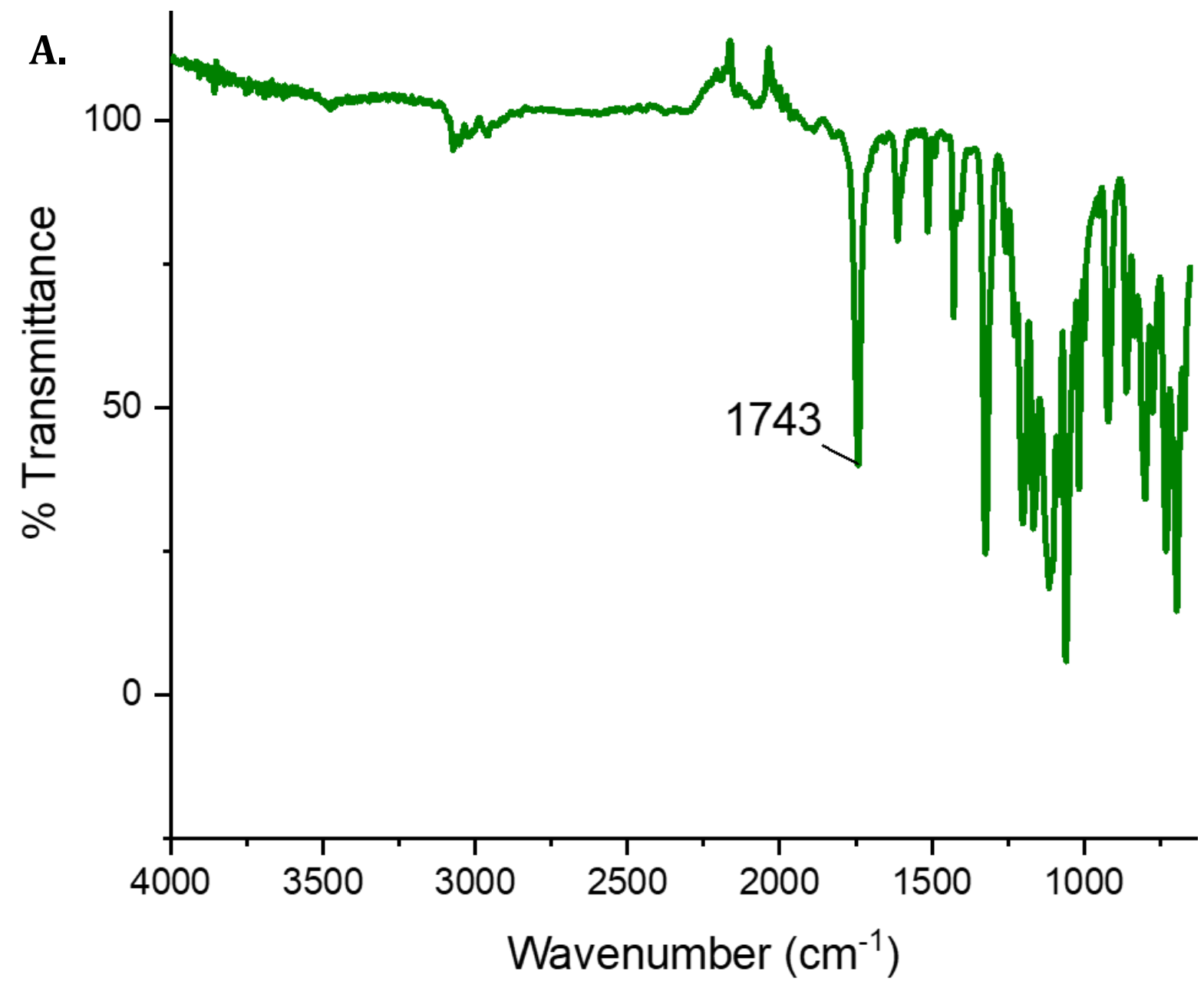


B.

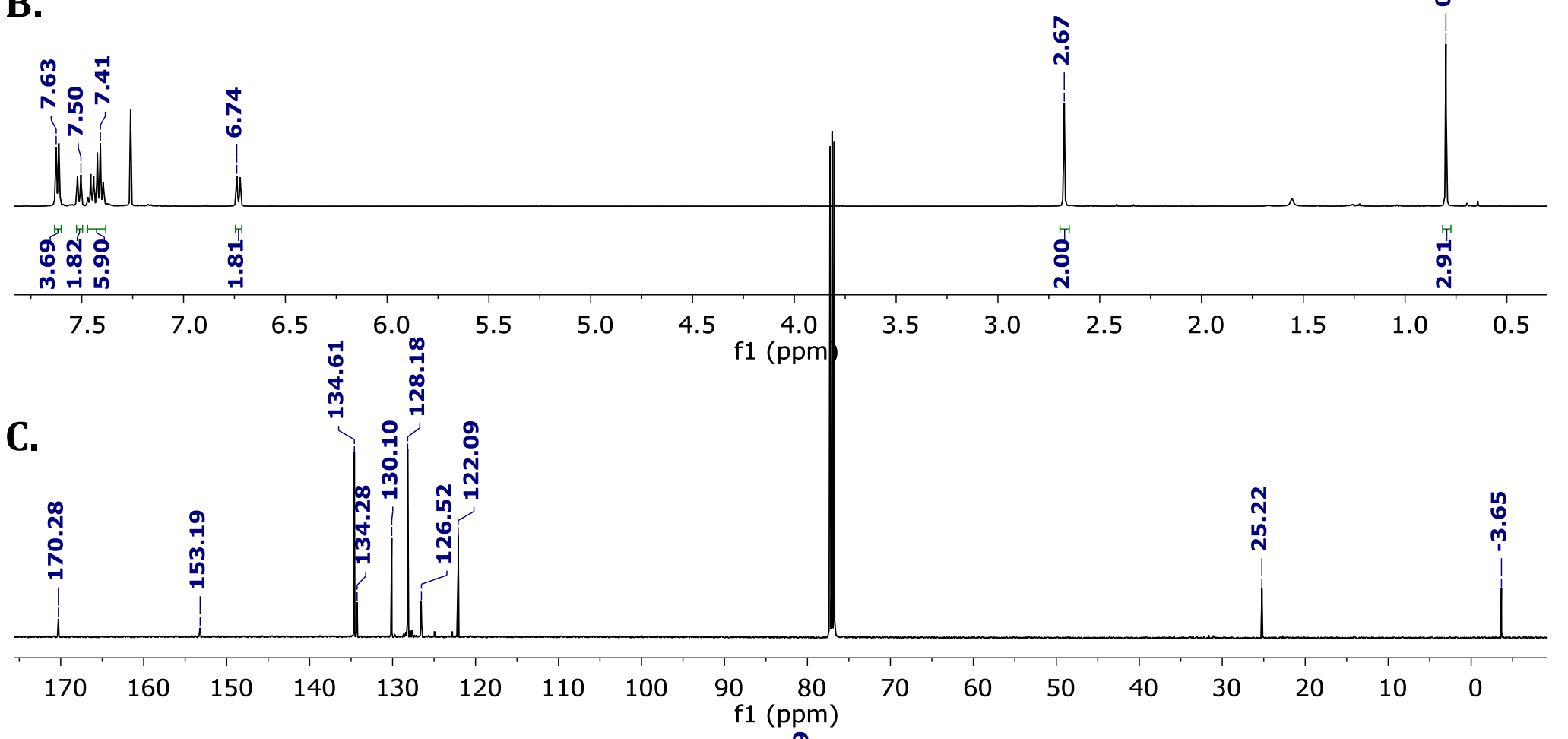

D.
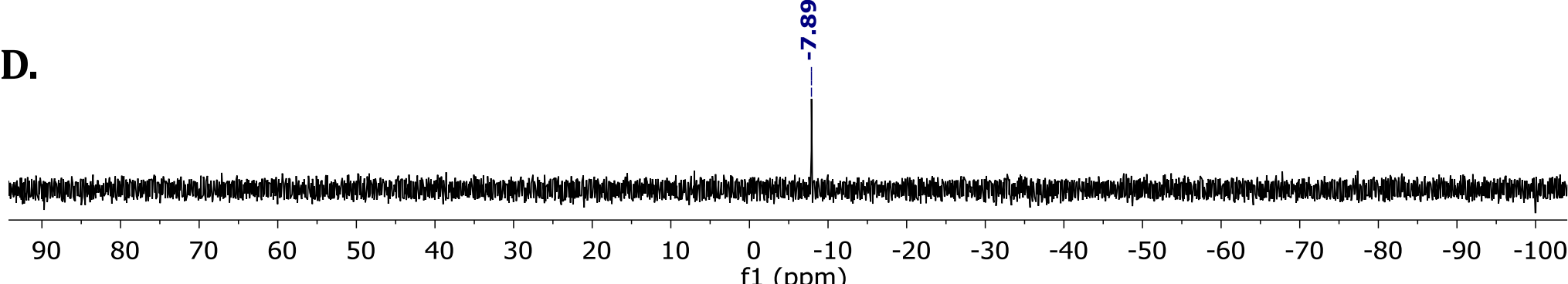
E.

09191913b \#102-110 RT: 0.45-0.49 AV: 9 SB: 24 0.32-0.43 NL: 2.76E7 T: FTMS + p ESI Full ms [50.0000-700.0000]

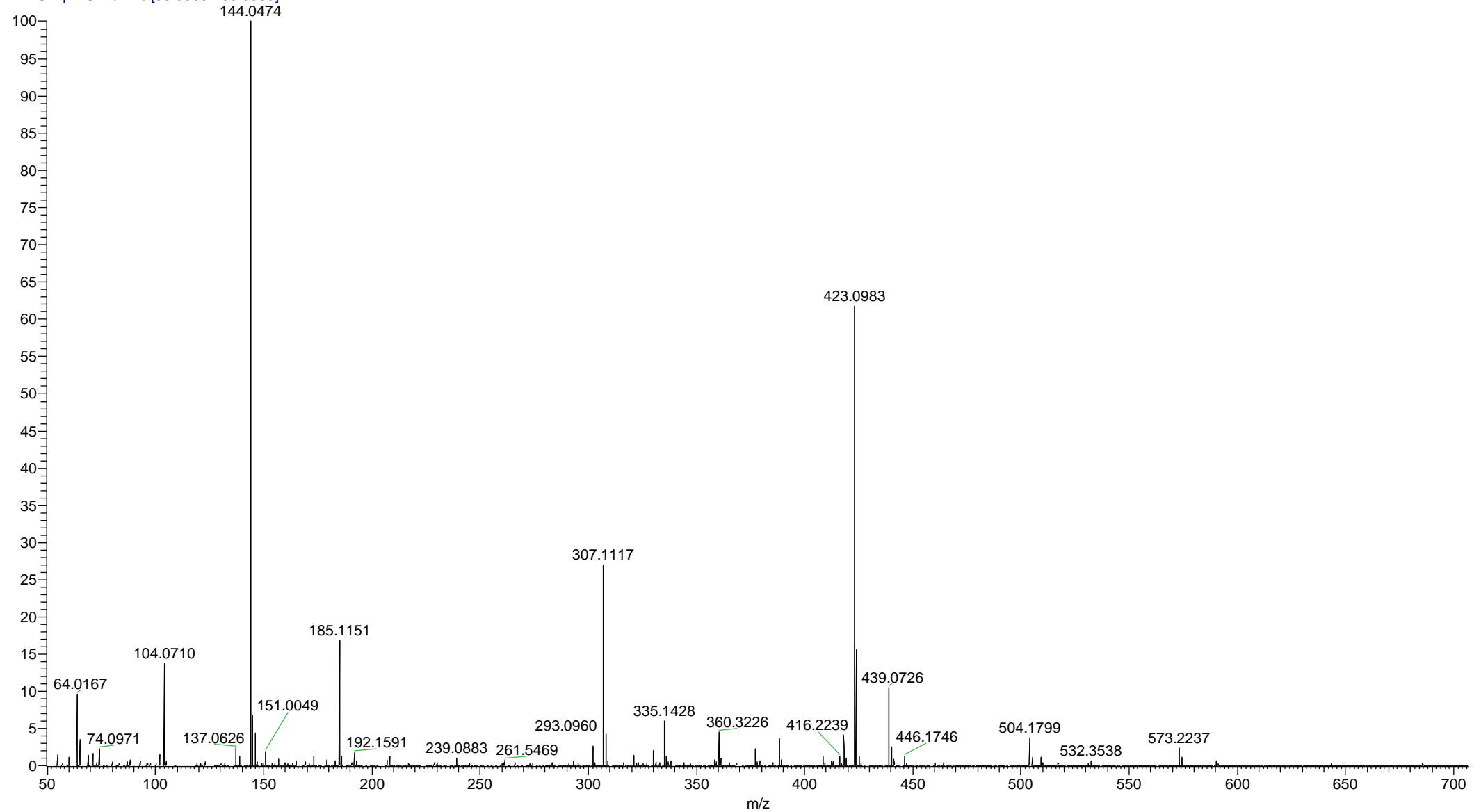


Figure S14. Compound 9 (A) FTIR (B) ${ }^{1} \mathrm{H}$ NMR (C) ${ }^{13} \mathrm{C}$ NMR (D) ${ }^{29}$ Si NMR (E) mass spectroscopy<smiles>CC(=O)OCc1cccc(OC(=O)C[Si](C)(c2ccccc2)c2ccccc2)c1</smiles>

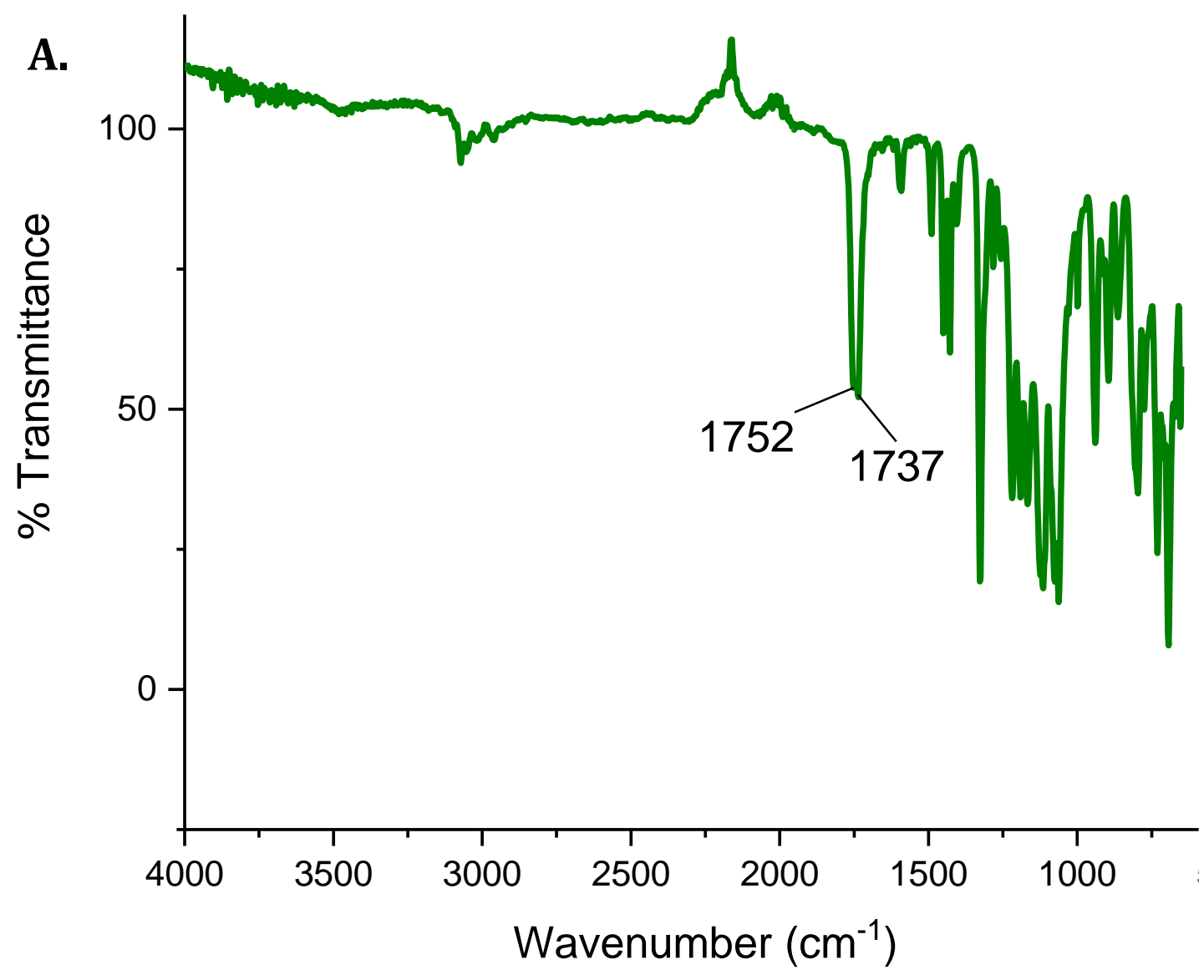




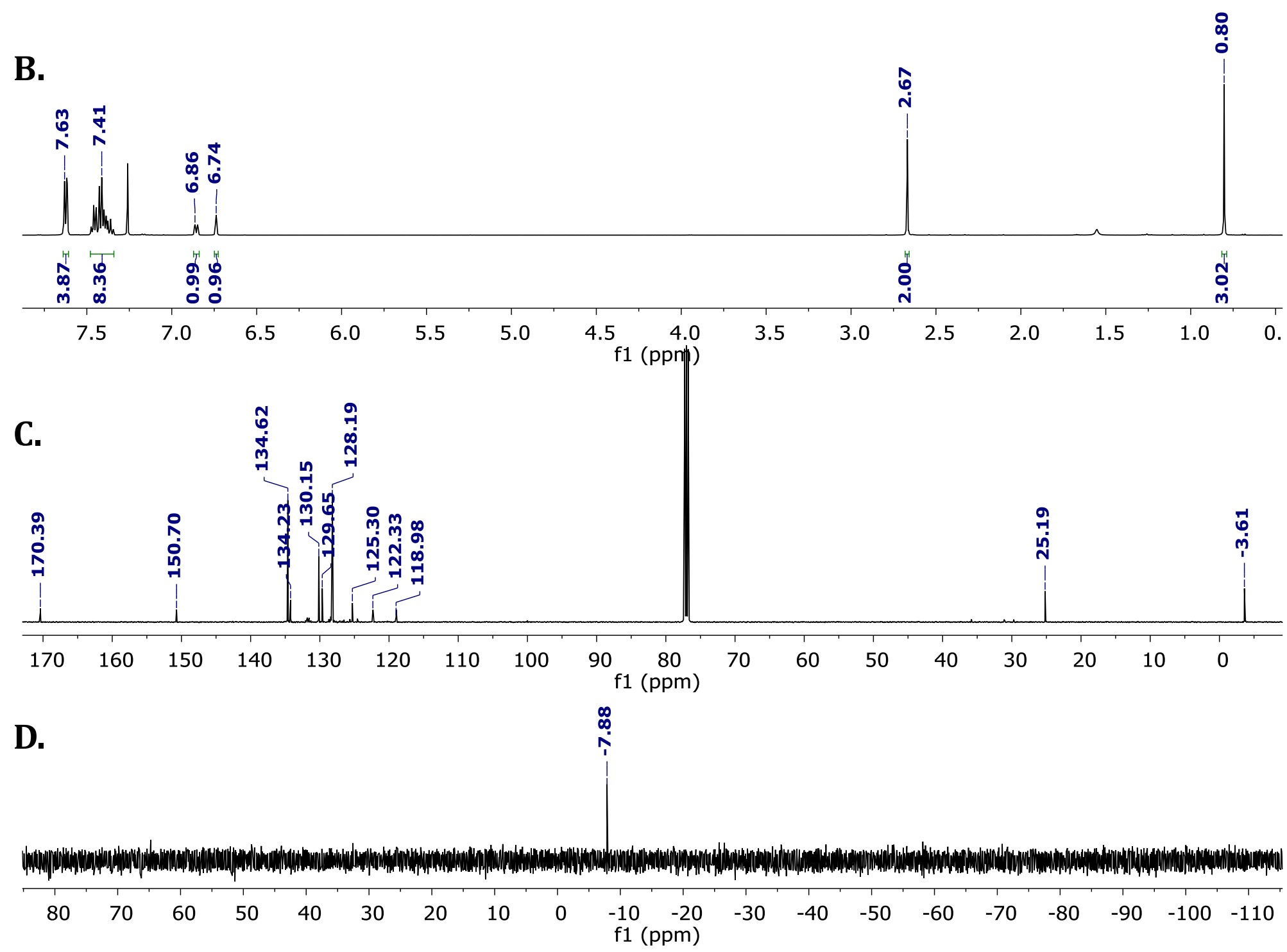


E.

09191914b \#104-112 RT: 0.46-0.50 AV: 9 SB: 16 0.25-0.32 NL: 4.59E7 T: FTMS + p ESI Full ms [50.0000-700.0000]

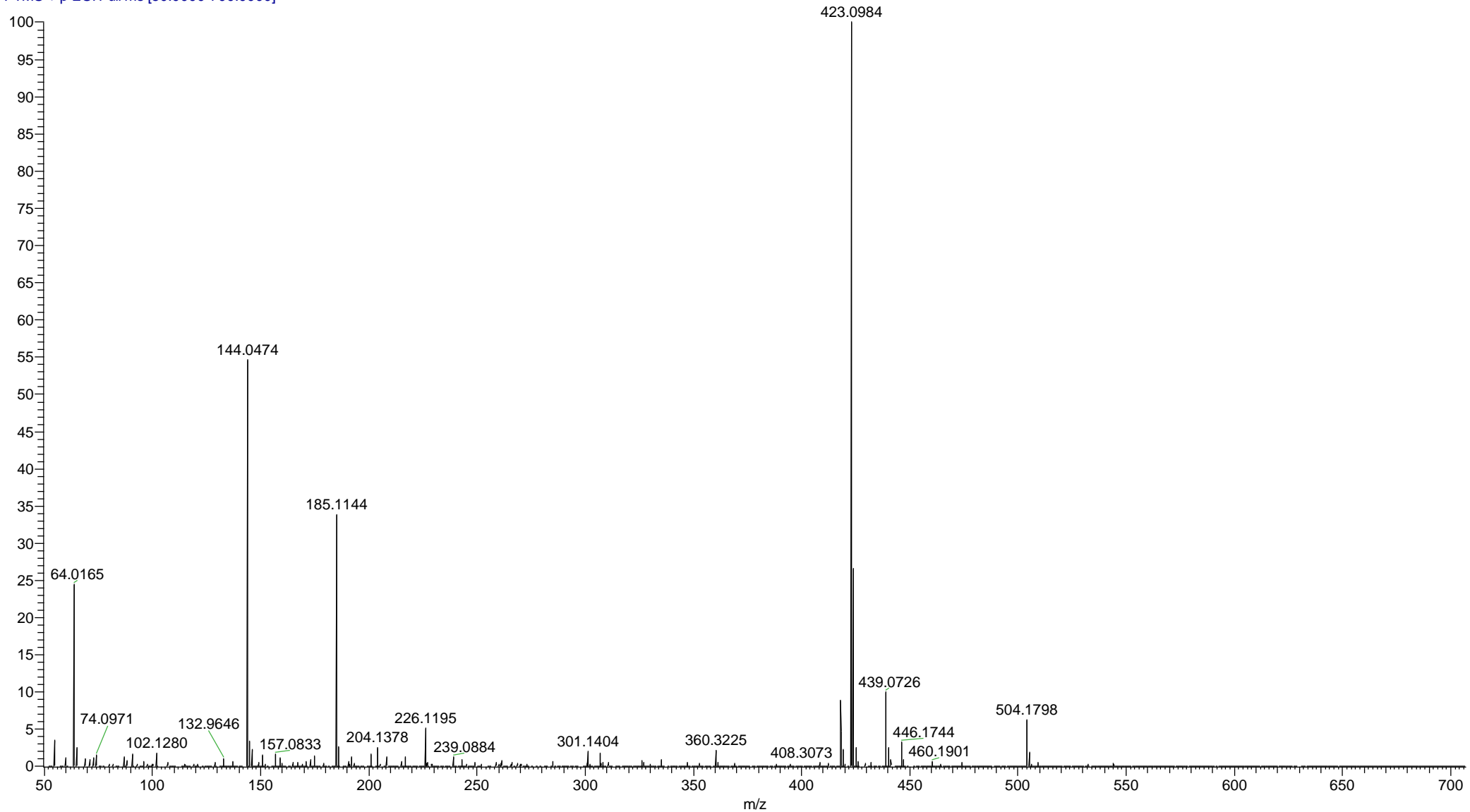


Figure S15. Compound 10 (A) FTIR (B) ${ }^{1} \mathrm{H}$ NMR (C) ${ }^{13} \mathrm{C}$ NMR (D) ${ }^{29}$ Si NMR (E) mass spectroscopy<smiles>CC(=O)O[Na]</smiles>

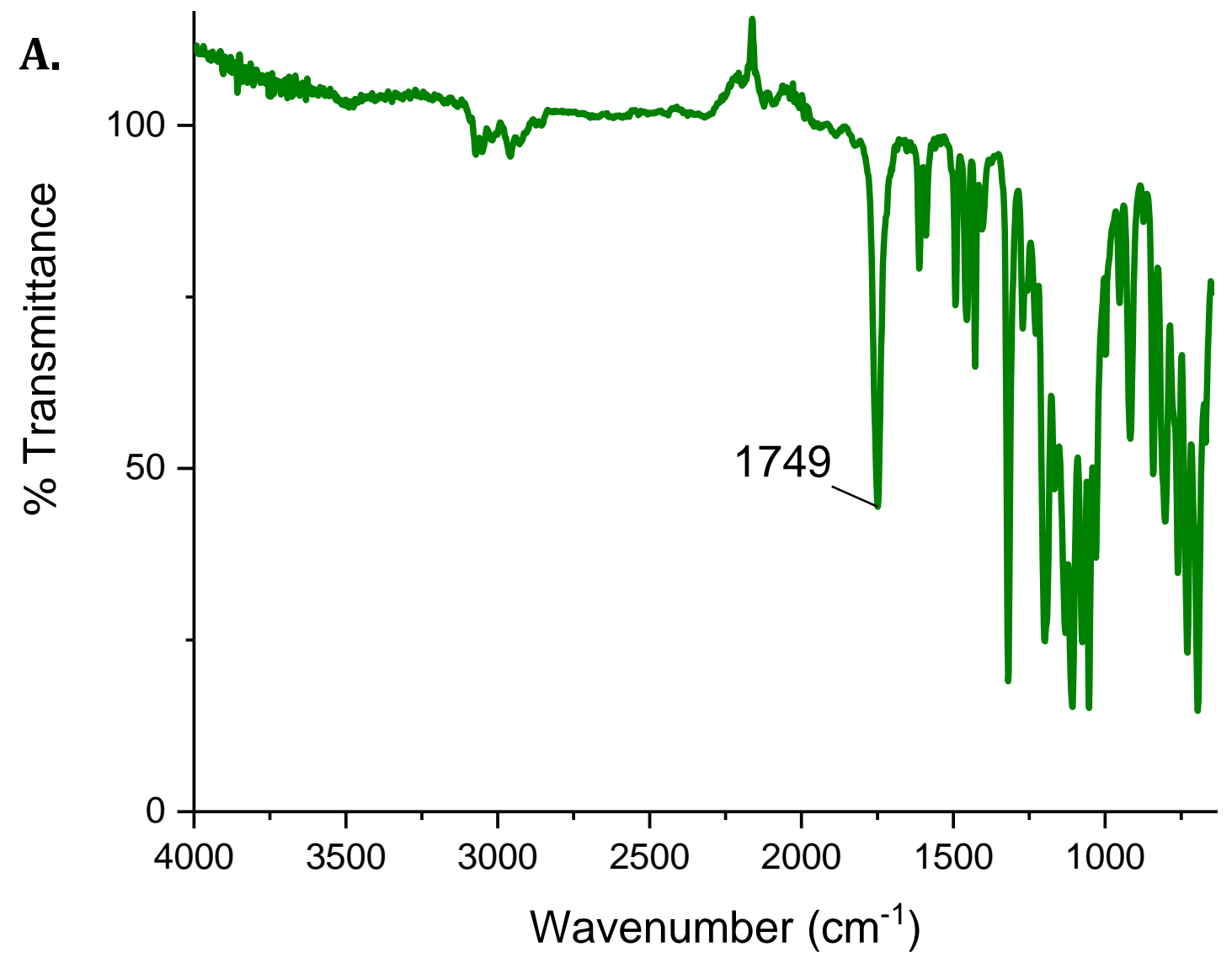


B.

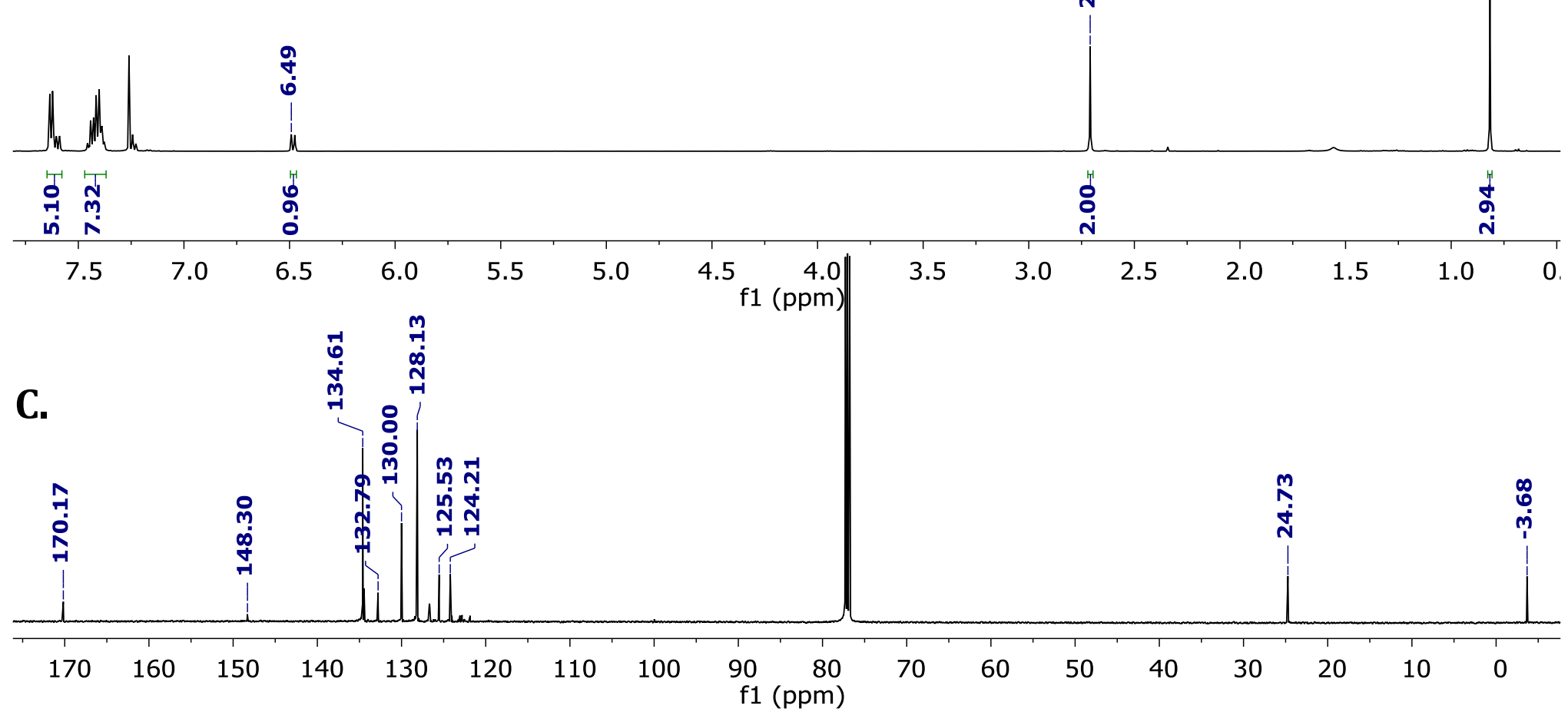

D.

$\stackrel{\text { oे }}{\infty}$

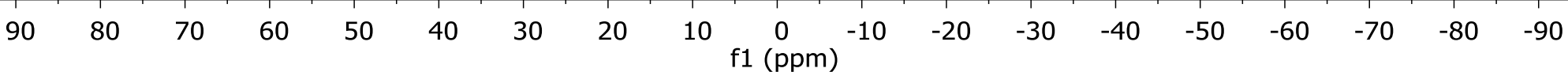


E.

09191915 \#109-116 RT: 0.49-0.52 AV: 8 SB: 25 0.30-0.41 NL: 6.94E7 T: FTMS + p ESI Full ms [50.0000-700.0000]

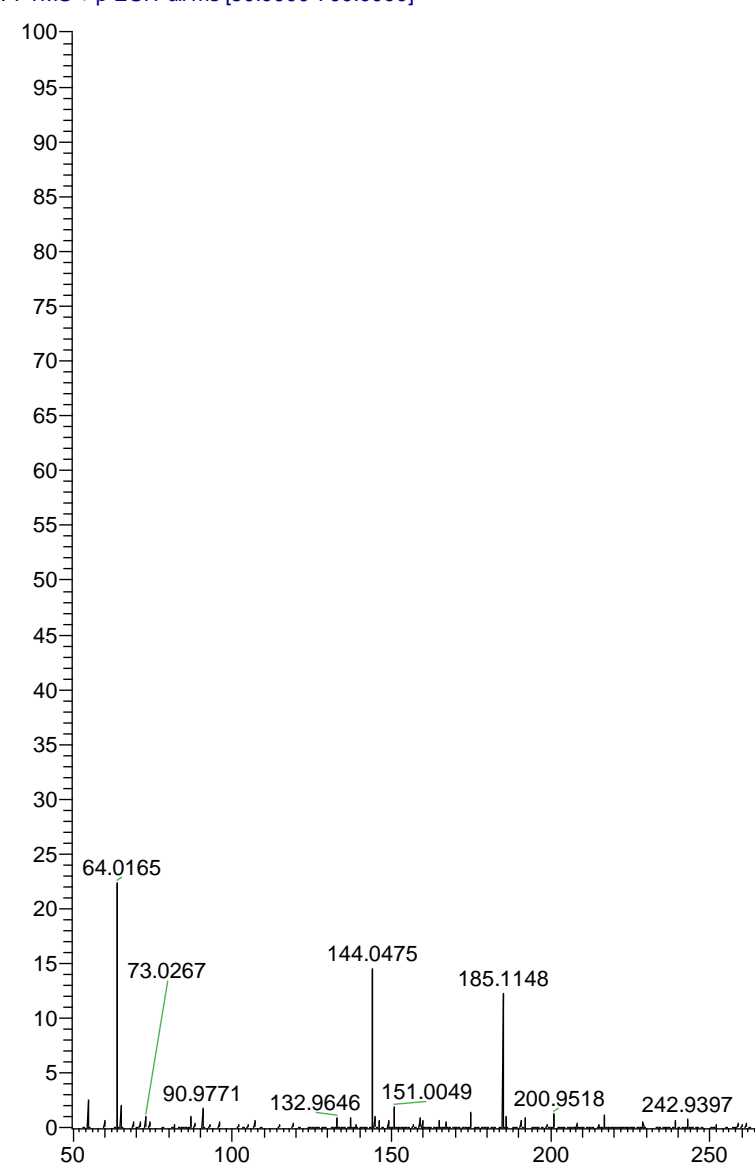

423.0983

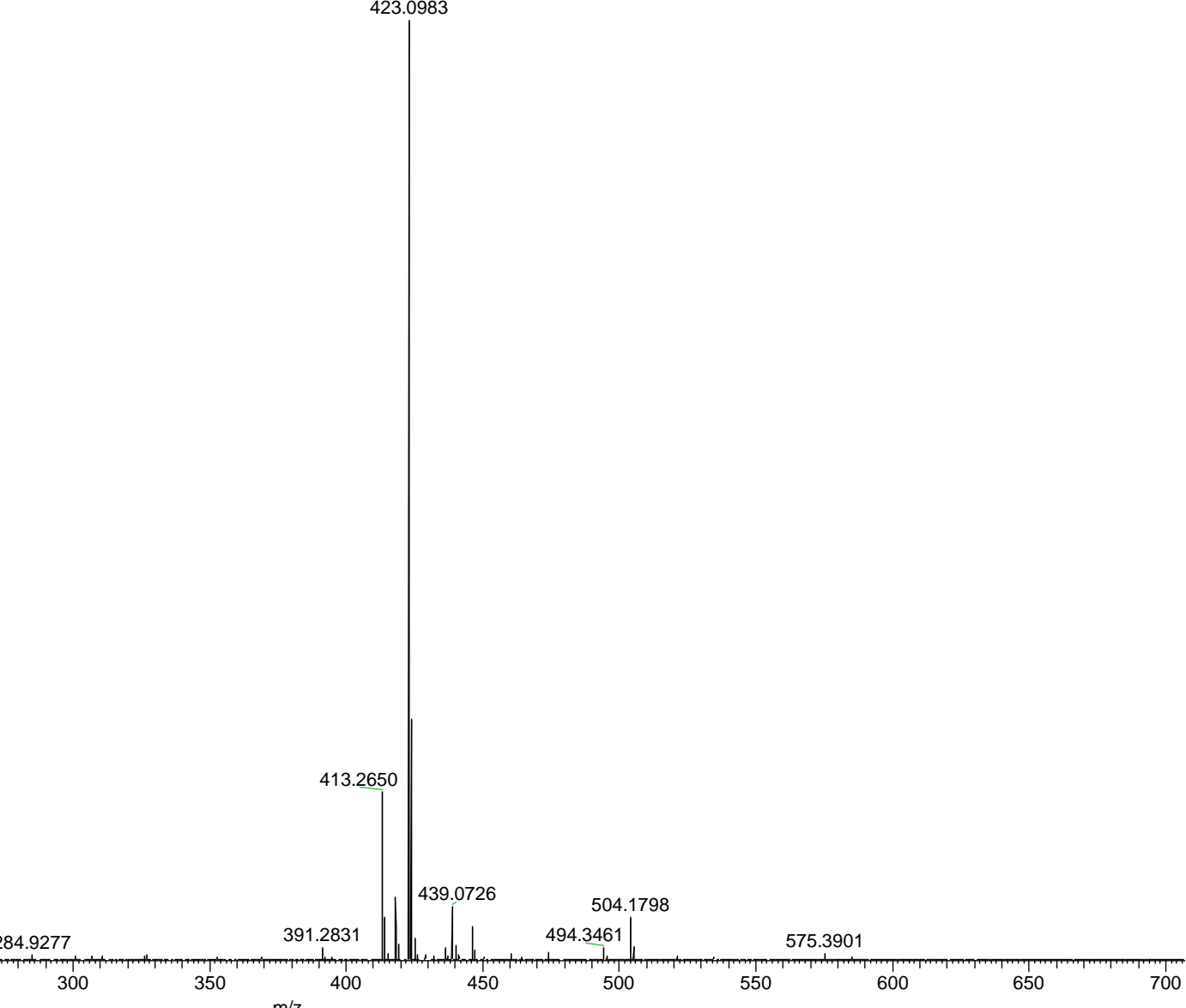


Figure S16. Compound 11 (A) FTIR (B) ${ }^{1} \mathrm{H}$ NMR (C) ${ }^{13} \mathrm{C}$ NMR (D) ${ }^{29}$ Si NMR (E) mass spectroscopy<smiles>C[Si](C)(C)N=[W]</smiles>

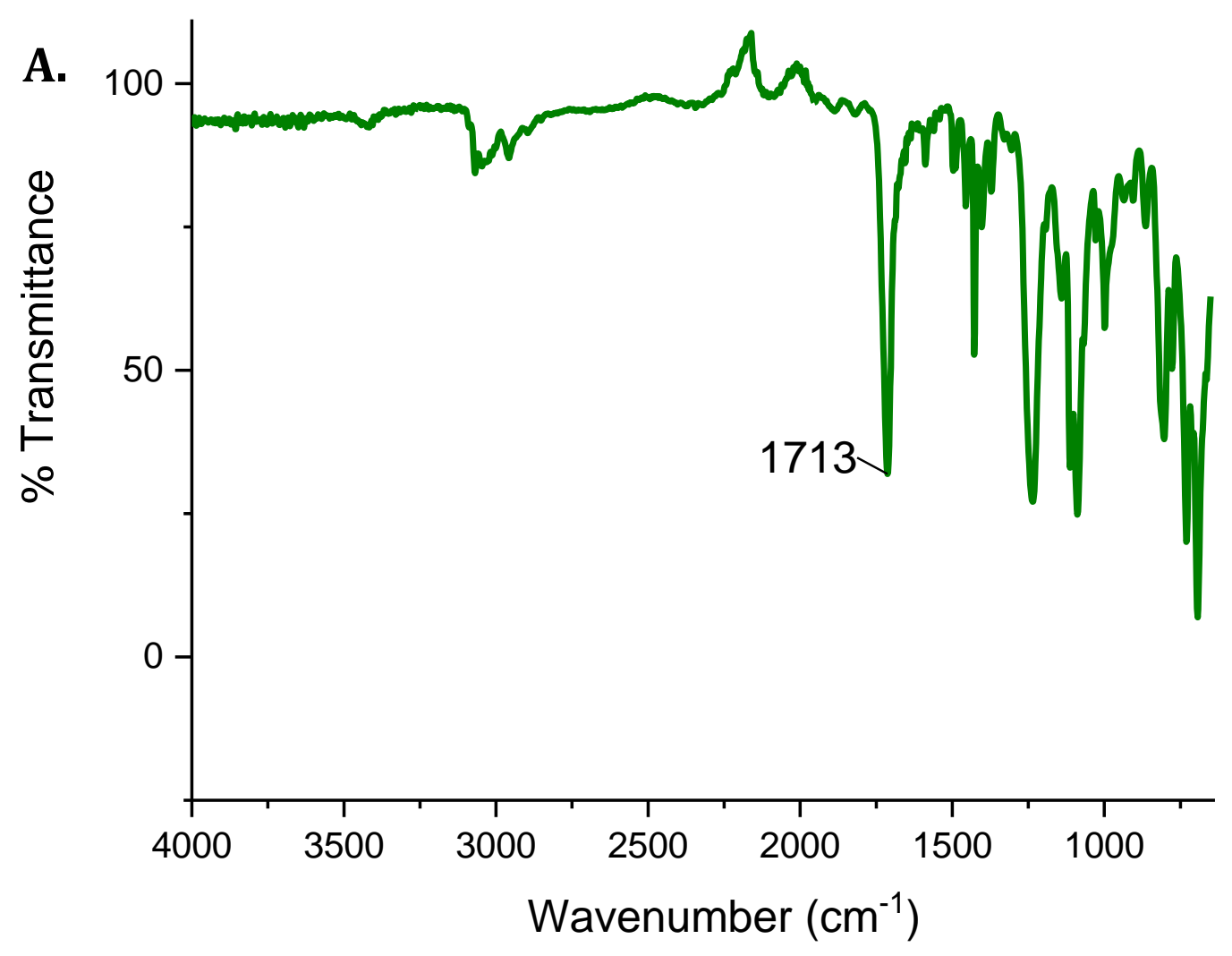




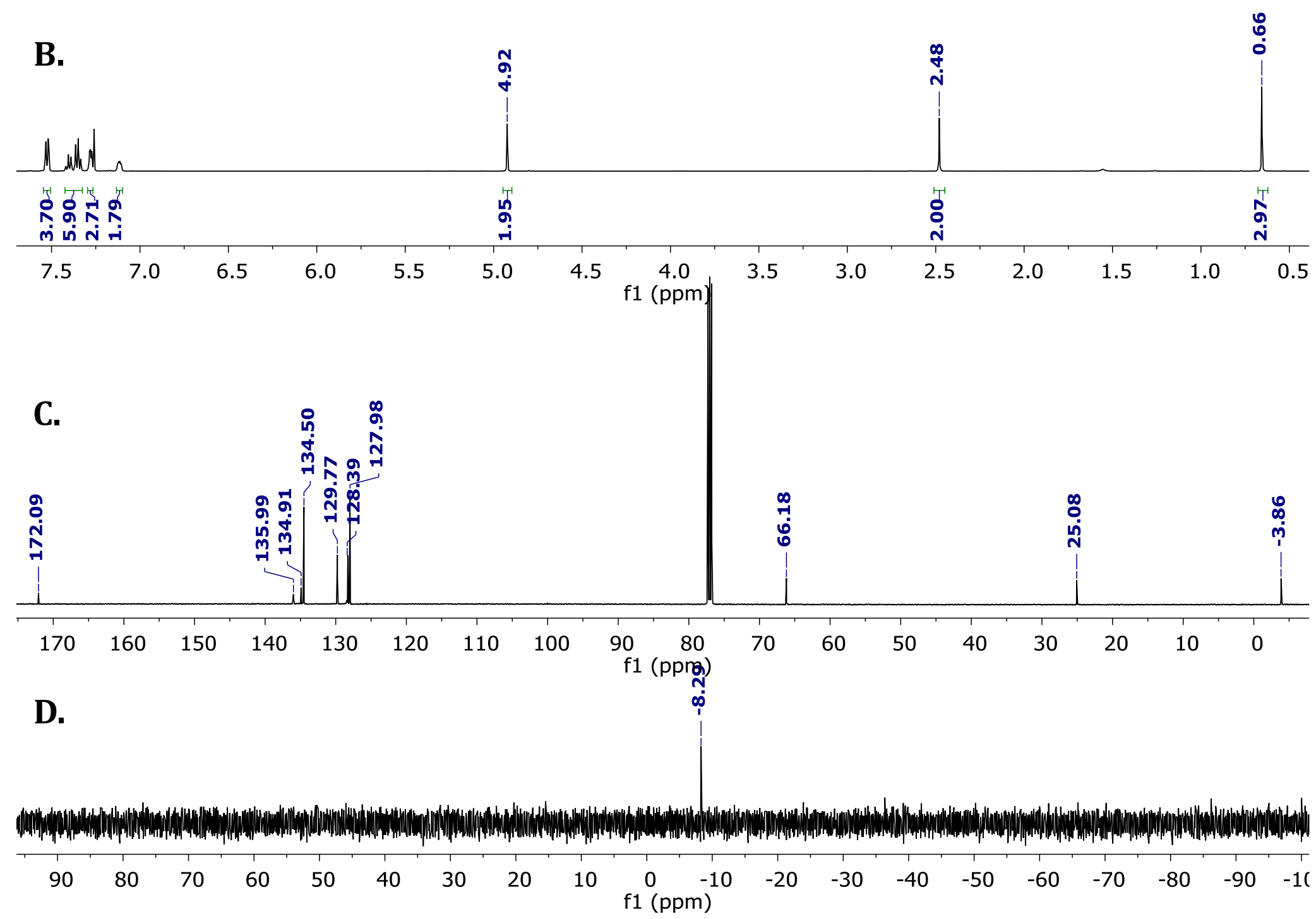


E. $09191916 \# 114-124 \quad$ RT: $0.51-0.55 \quad$ AV: 11 SB: 20 0.34-0.43 NL: 6.33E7
T: FTMS + p ESI Full ms [50.0000-500.0000]

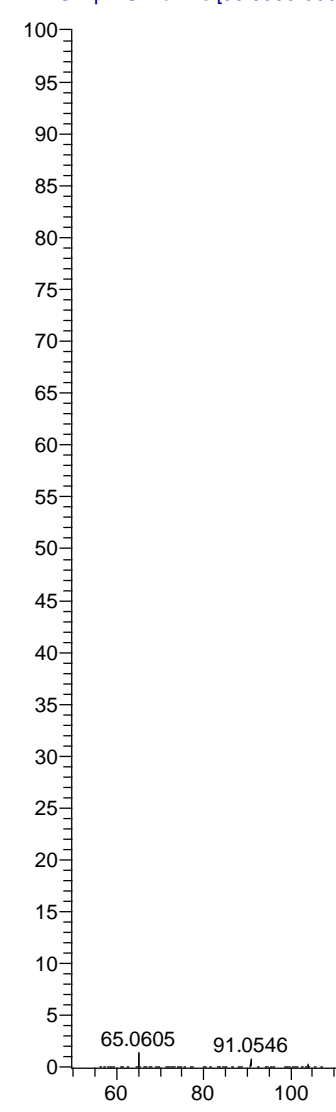

$$
11 \text { SB: } 20 \text { 0.34-0.43 NL: 6.33E7 }
$$


Figure S17. Compound 12 (A) FTIR (B) ${ }^{1} \mathrm{H}$ NMR (C) ${ }^{13} \mathrm{C}$ NMR (D) ${ }^{29}$ Si NMR (E) mass spectroscopy<smiles>CCCNC(=O)C[Si](C)(c1ccccc1)c1ccccc1</smiles>

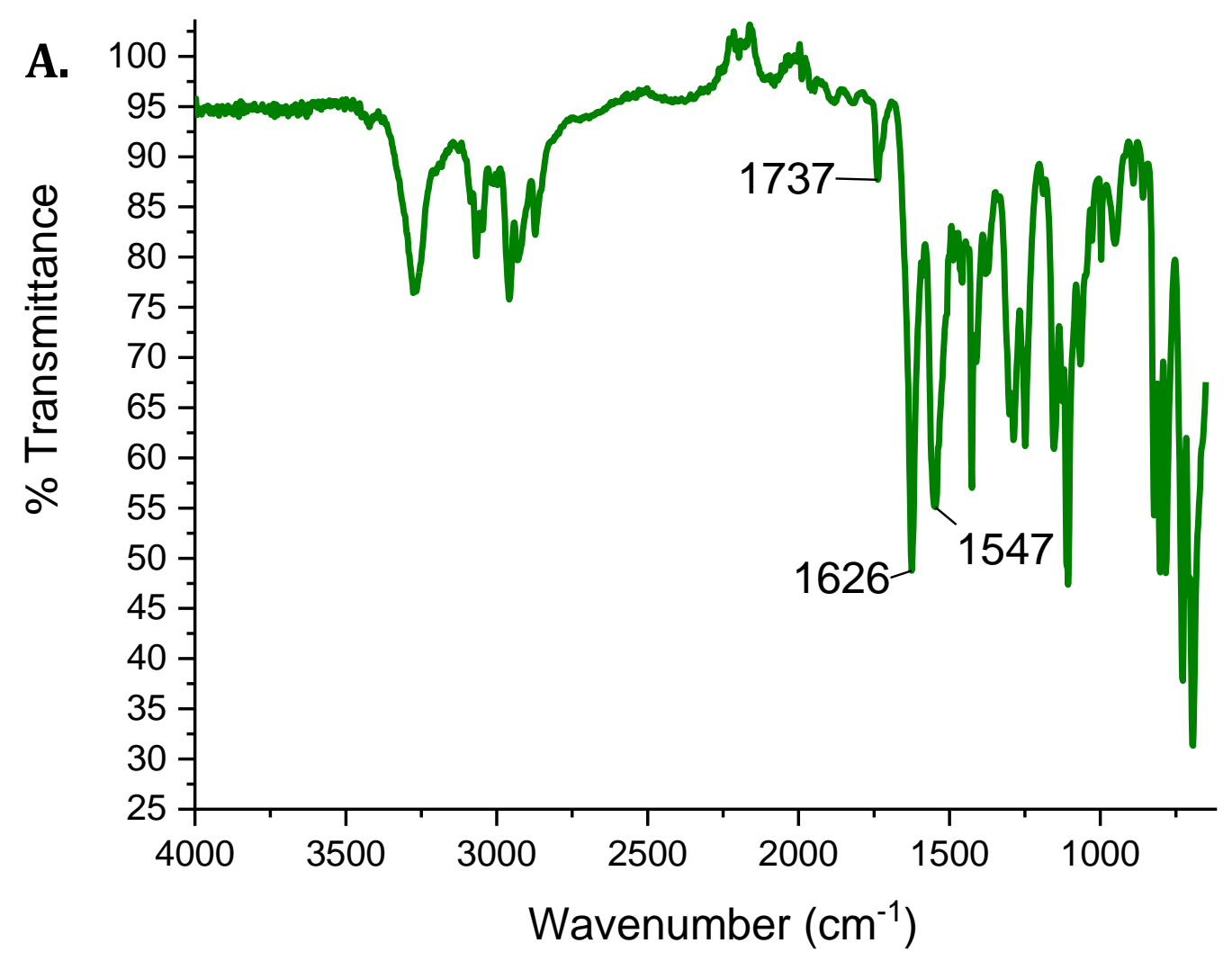




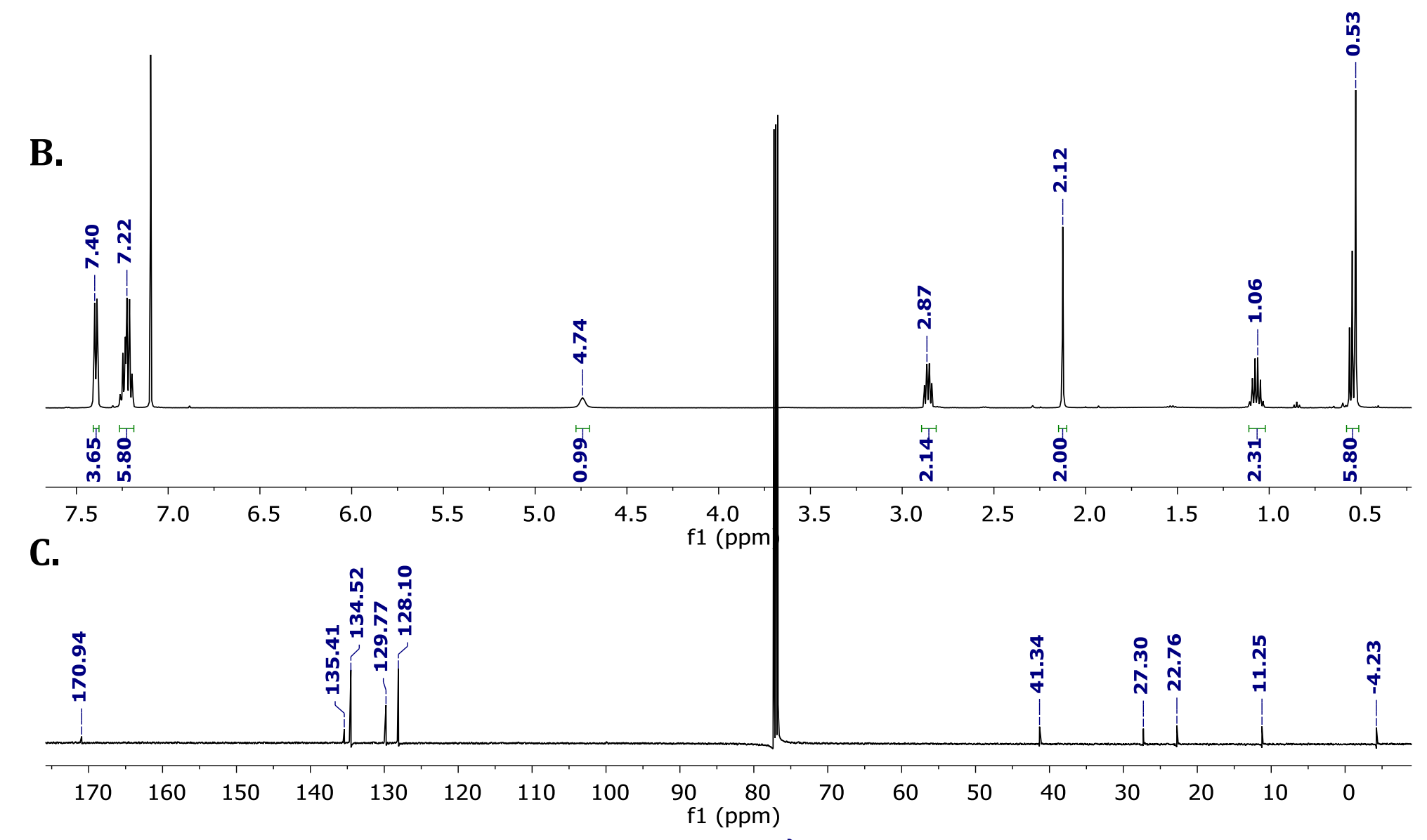

D.

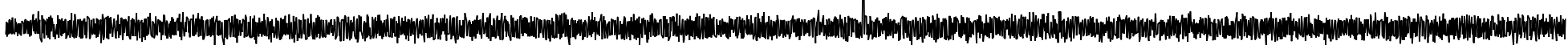

$\begin{array}{lllllllllllllllllll}90 & 80 & 70 & 60 & 50 & 40 & 30 & 20 & 10 \underset{f 1(\mathrm{ppm})}{0}-10 & -20 & -30 & -40 & -50 & -60 & -70 & -80 & -90\end{array}$


E.

09231913 \#90-94 RT: 0.40-0.42 AV: 5 SB: 22 0.30-0.39 NL: 2.05E8 T: FTMS + p ESI Full ms [50.0000-500.0000]

100

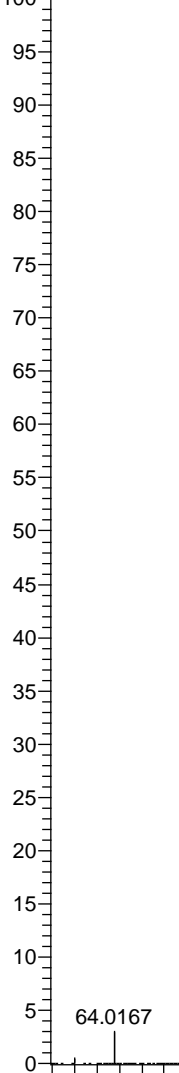

年

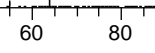

320.1435

326.3776

298.1617

343.2195

343.2195

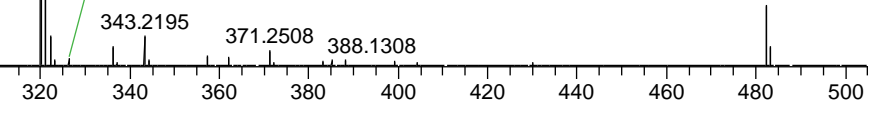

482.2690 
Figure S18. Compound 13 (A) FTIR (B) ${ }^{1} \mathrm{H}$ NMR (C) ${ }^{13} \mathrm{C}$ NMR (D) ${ }^{29}$ Si NMR (E) mass spectroscopy<smiles>CCCCN(CCCC)C(=O)C[Si](C)(c1ccccc1)c1ccccc1</smiles>

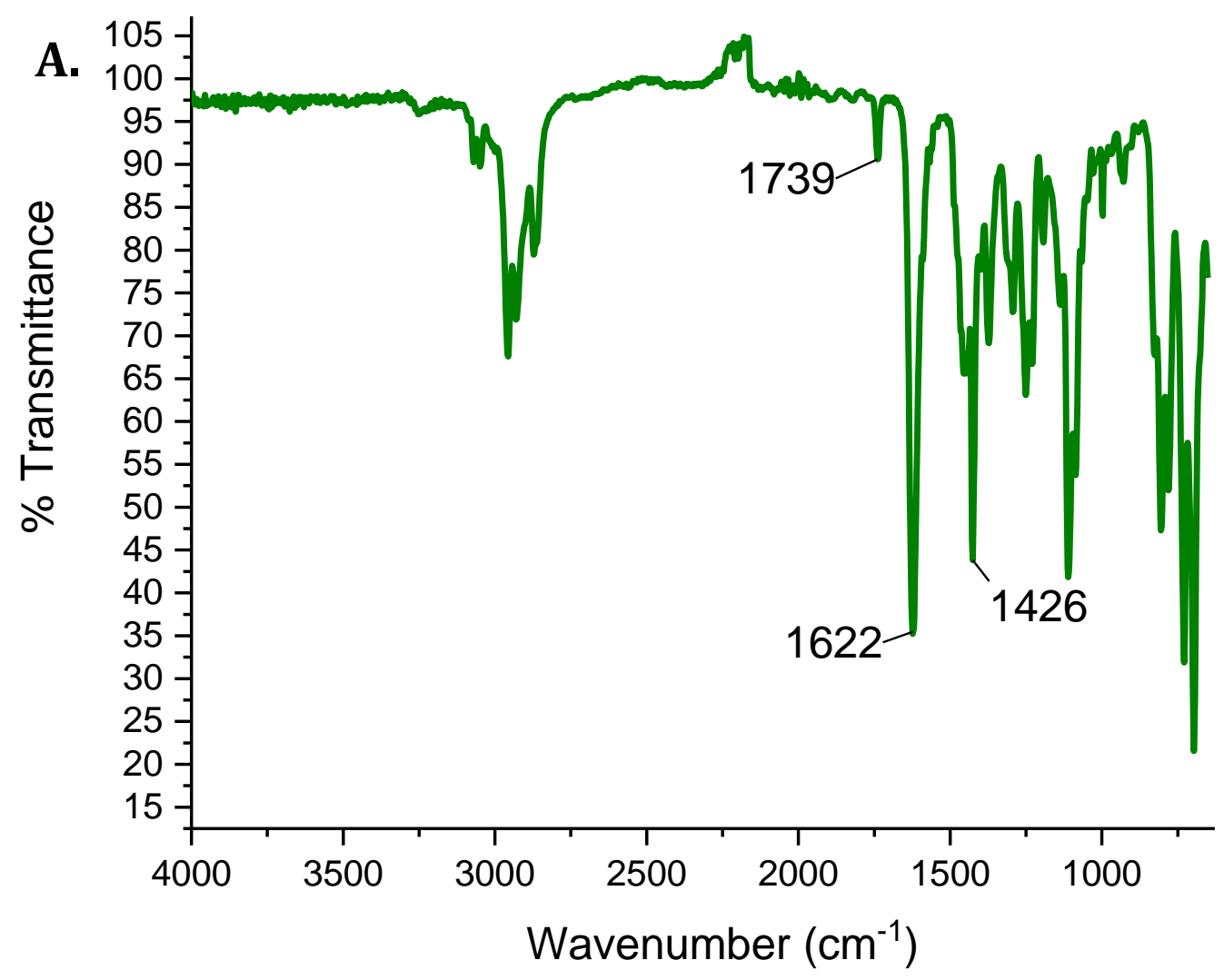




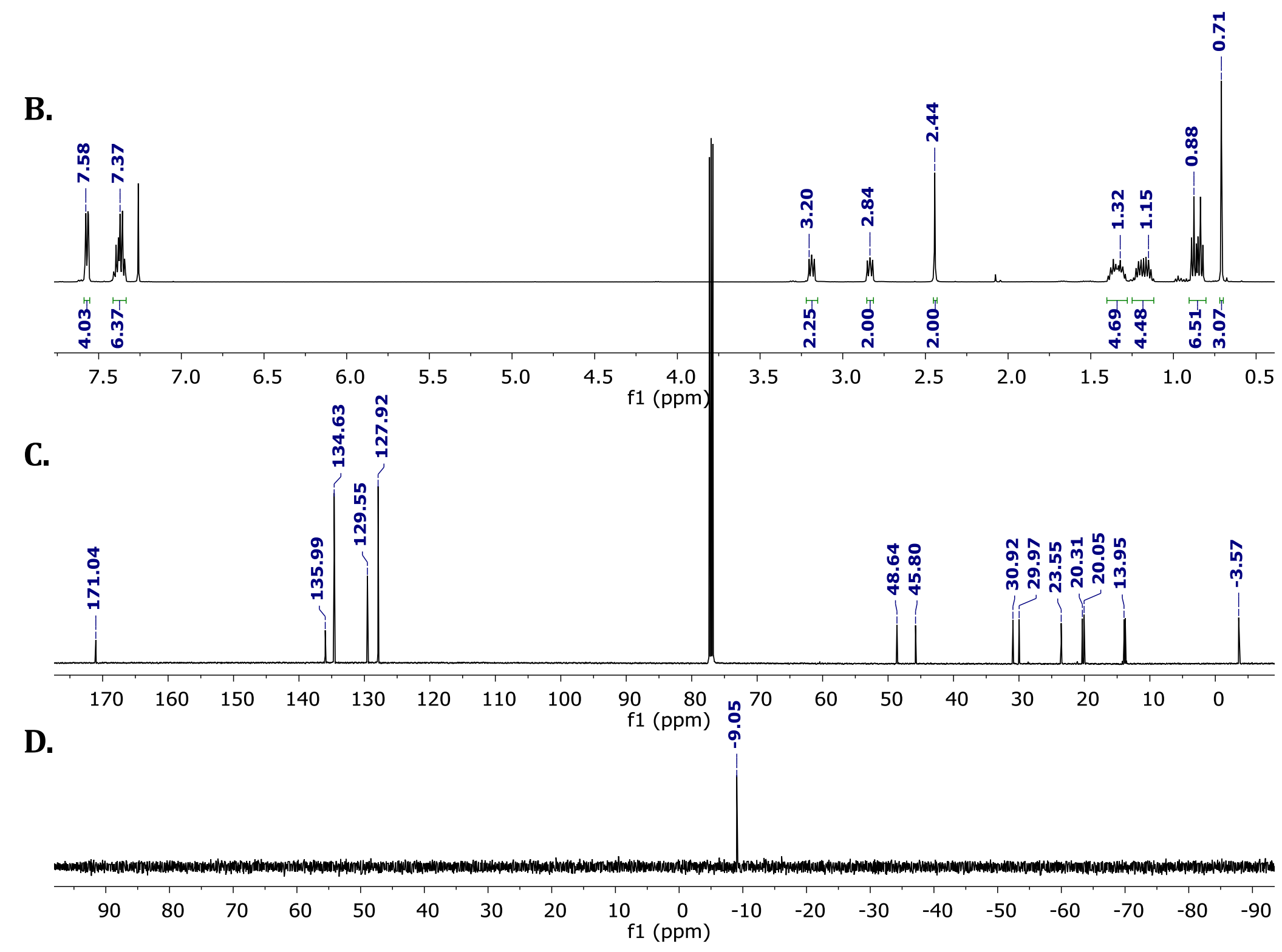




\section{E.}

Q3231914 A147-16. RT: $0.65-072$ AV 16 Se: 17 0.54-0.51 Ne: 29668

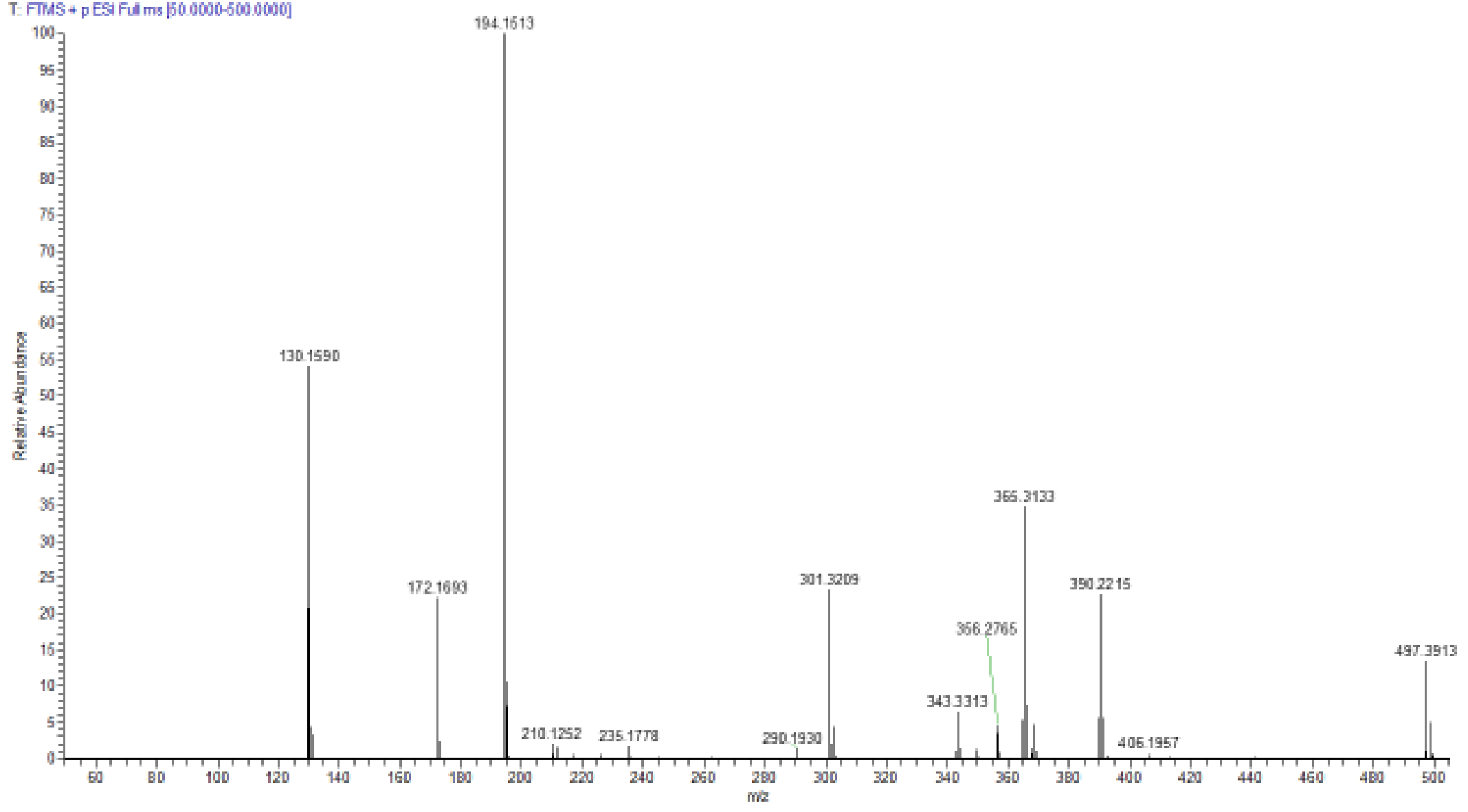


Figure S19. Compound 14 (A) FTIR (B) ${ }^{1} \mathrm{H}$ NMR (C) ${ }^{13} \mathrm{C}$ NMR (D) ${ }^{29}$ Si NMR (E) mass spectroscopy<smiles>C[Si](CC(=O)Nc1ccccc1)(c1ccccc1)c1ccccc1</smiles>

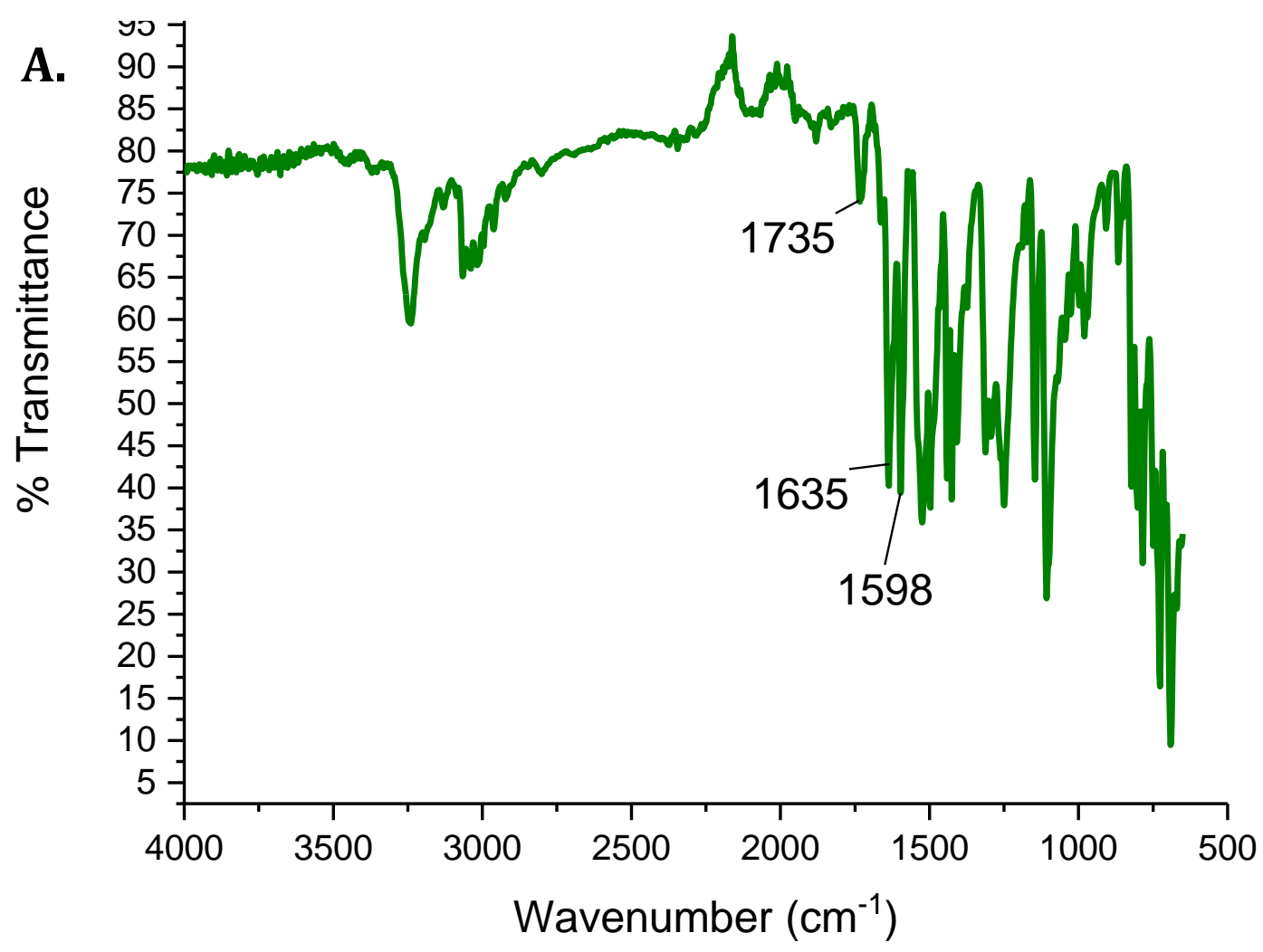




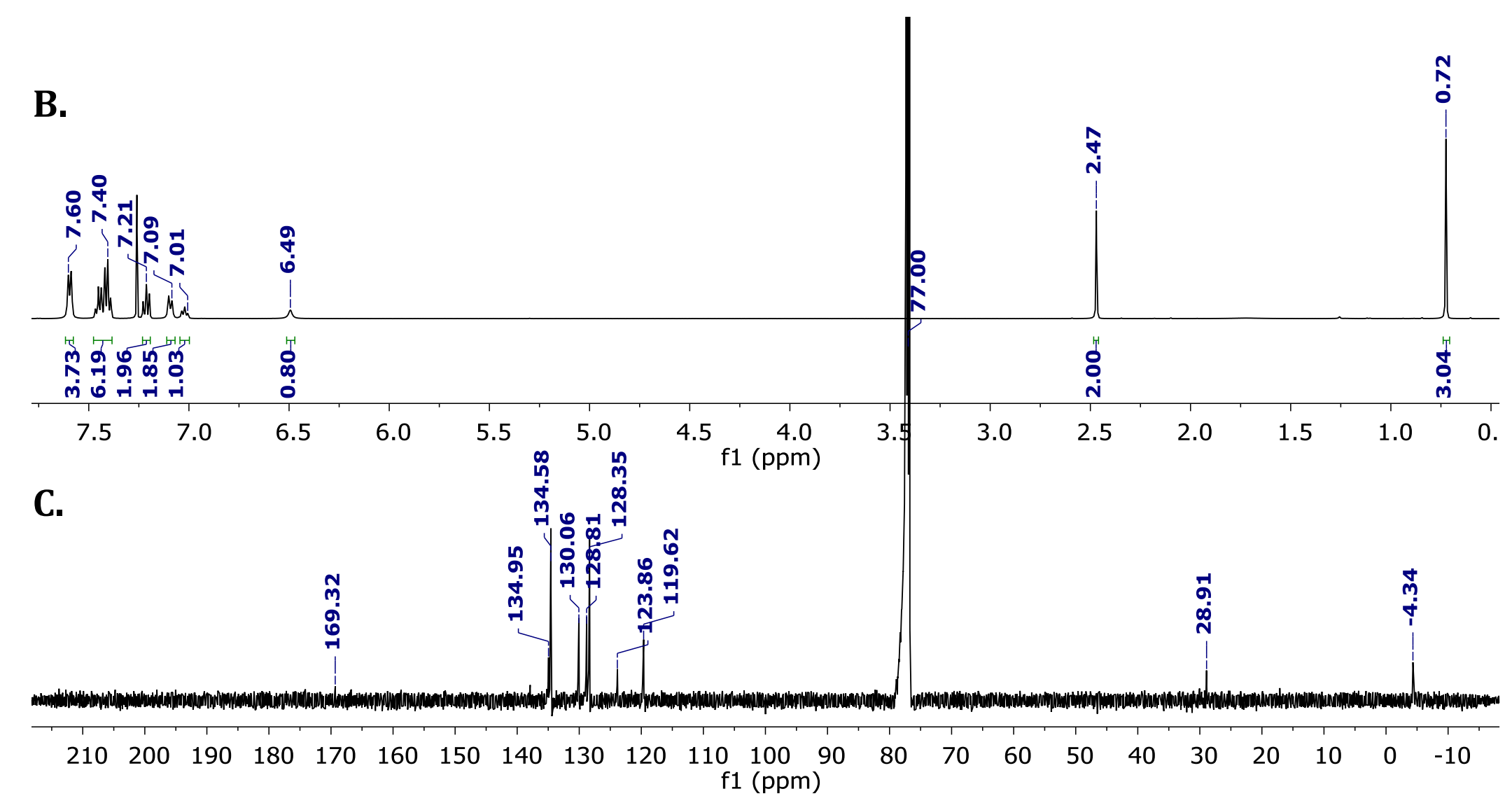

D.
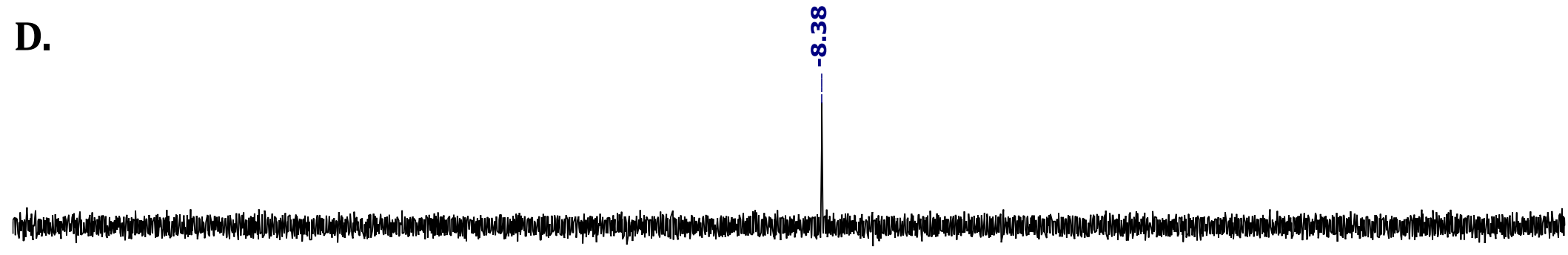
E.

09231915 \#102-110 RT: 0.45-0.49 AV: 9 SB: 22 0.32-0.42 NL: 8.29E7 T: FTMS + p ESI Full ms [50.0000-500.0000]

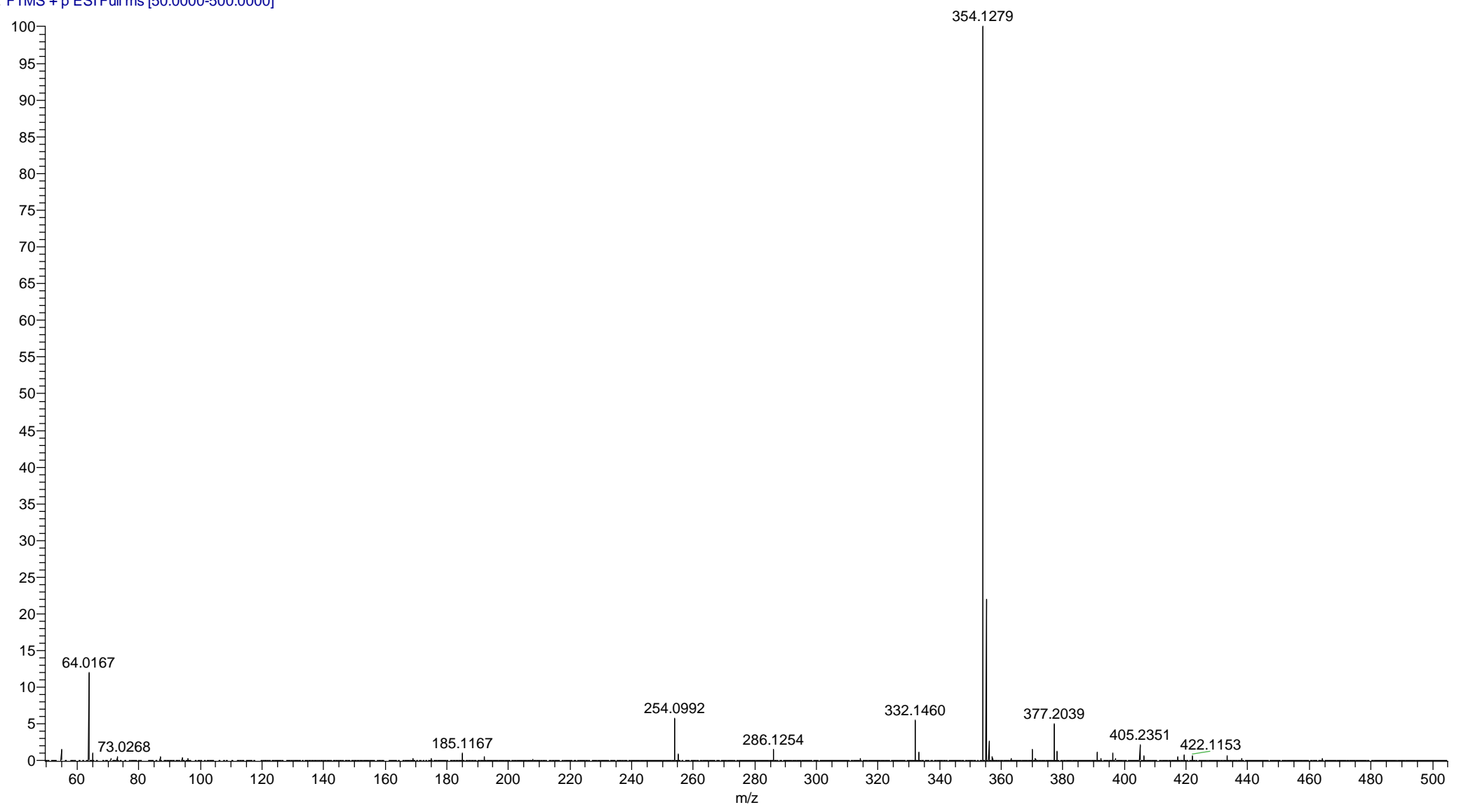


Figure S20. Compound 15 (A) FTIR (B) ${ }^{1} \mathrm{H}$ NMR (C) ${ }^{13} \mathrm{C}$ NMR (D) ${ }^{29}$ Si NMR (E) mass spectroscopy<smiles>CCSC(=O)C[Si](C)(c1ccccc1)c1ccccc1</smiles>

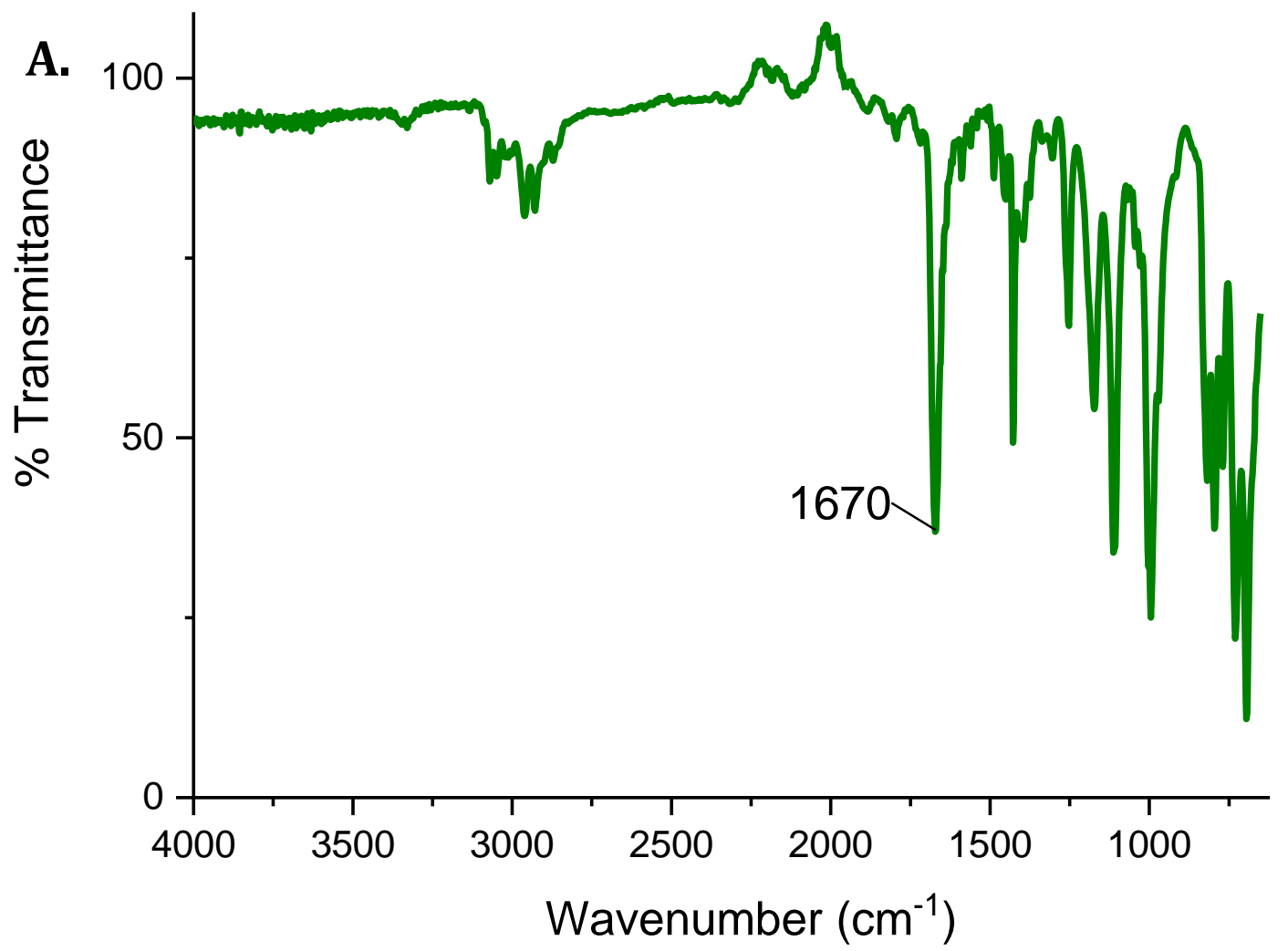




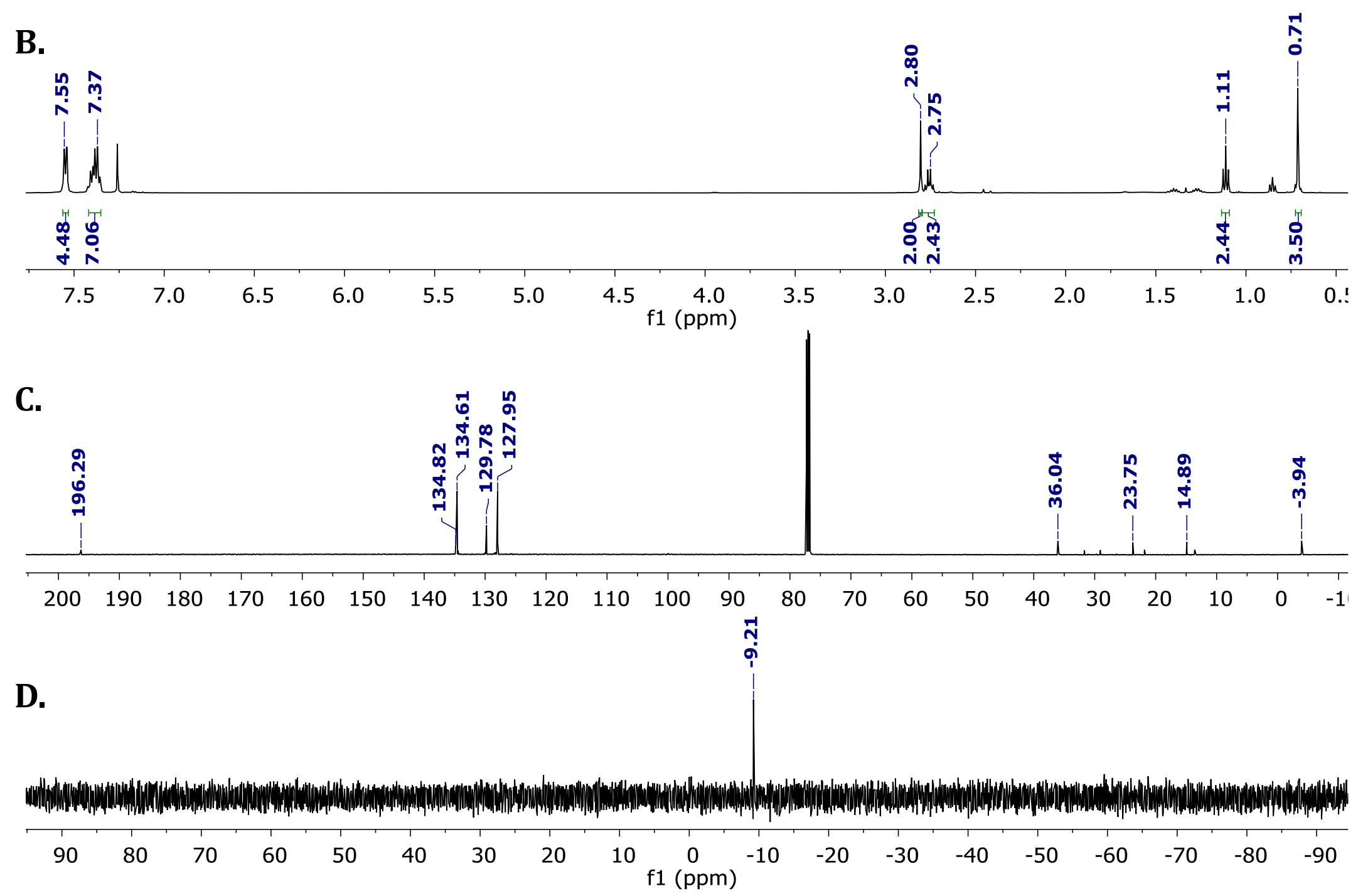


E.

09231901b \#158-162 RT: 0.70-0.72 AV: 5 SB: 13 0.50-0.55 NL: 2.47E7 T: FTMS + p ESI Full ms [50.0000-500.0000]

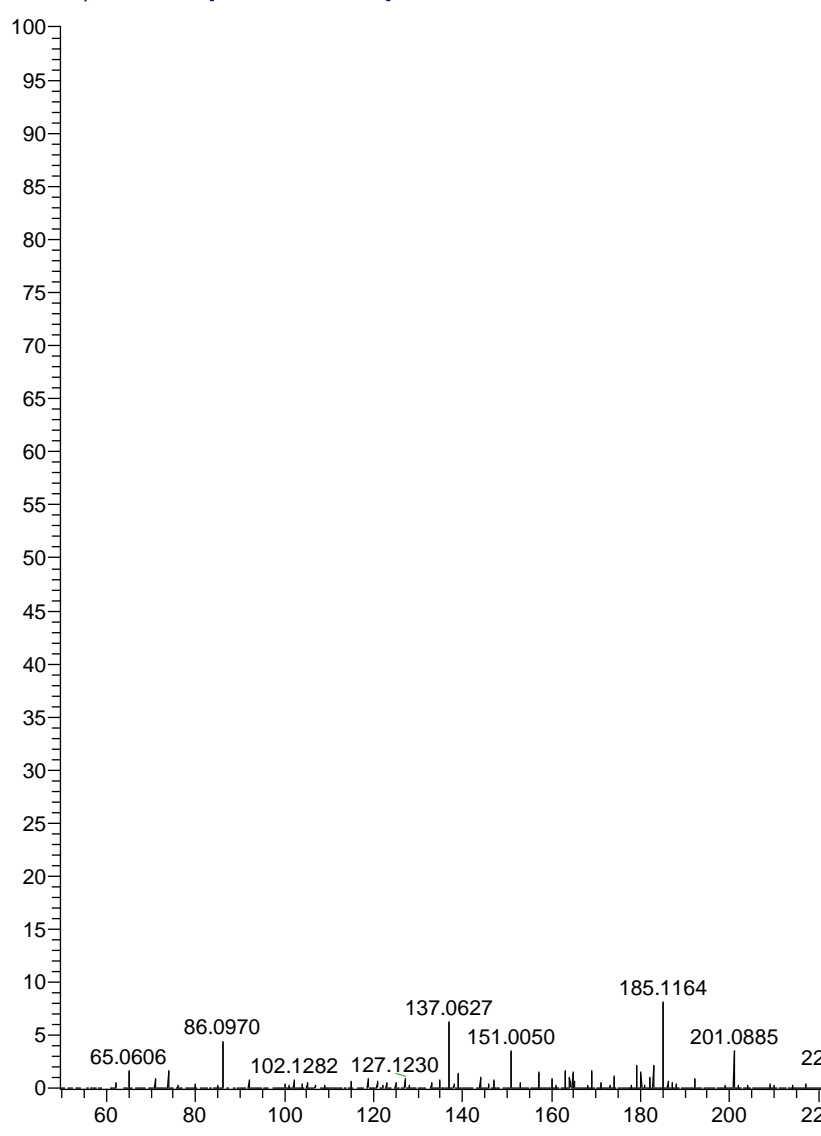

323.0890

351.1202

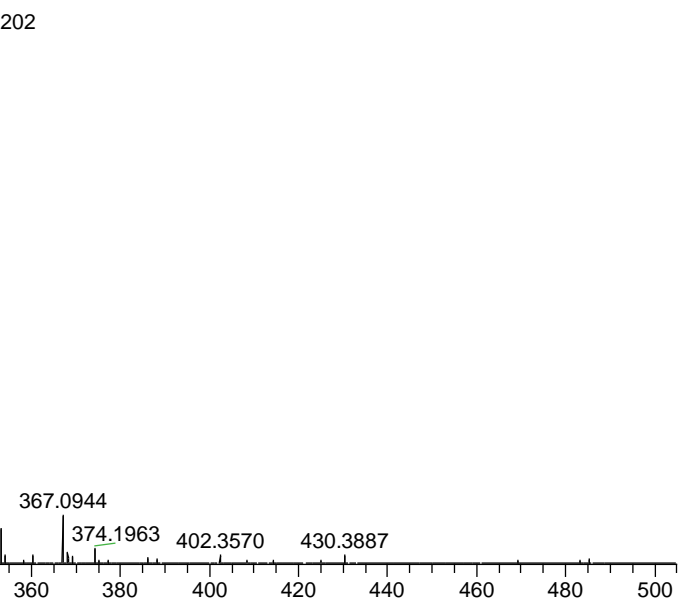


Figure S21. Compound 16 (A) FTIR (B) ${ }^{1} \mathrm{H}$ NMR (C) ${ }^{13} \mathrm{C}$ NMR (D) ${ }^{29}$ Si NMR (E) mass spectroscopy<smiles>CC(C)SC(=O)C[Si](C)(c1ccccc1)c1ccccc1</smiles>

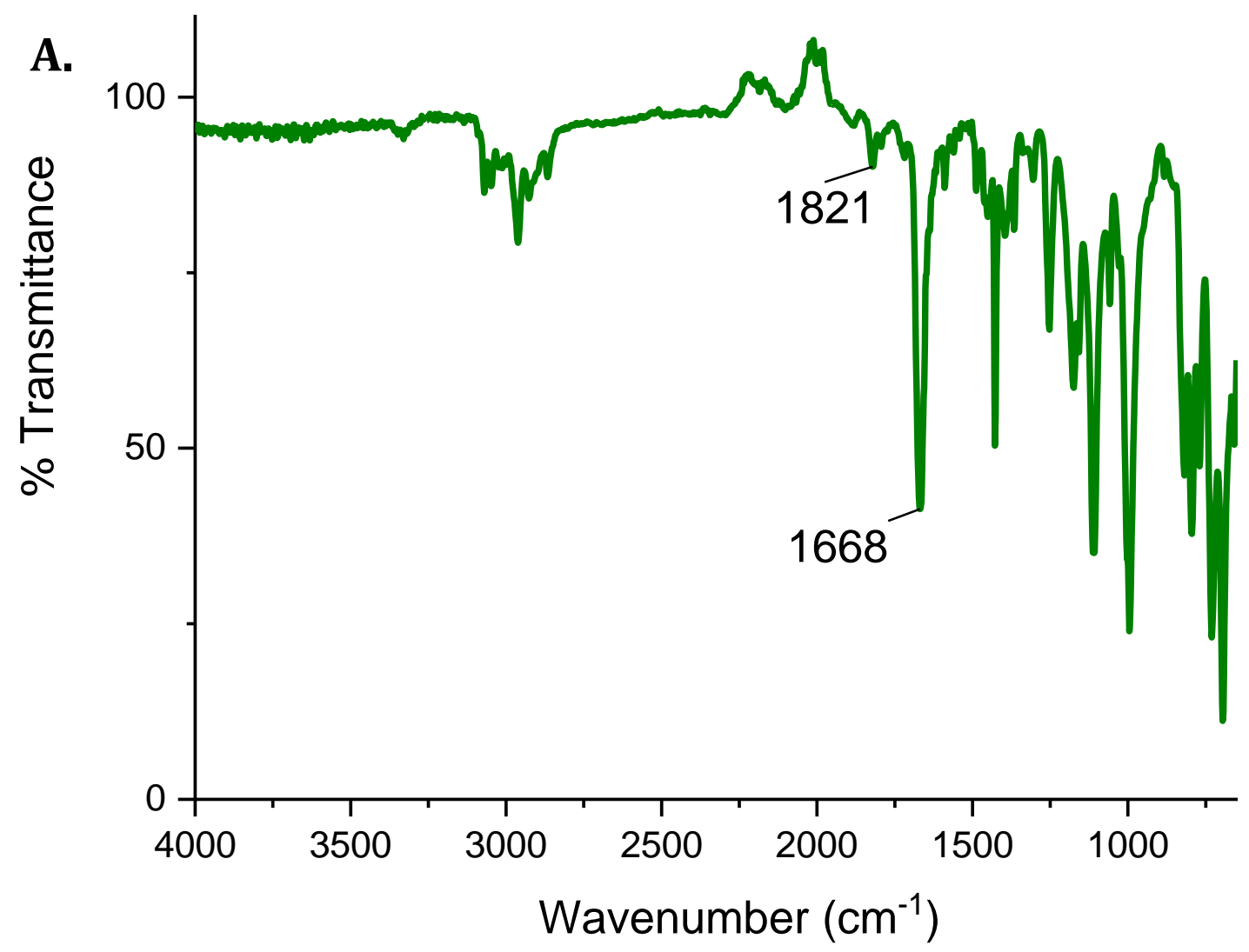




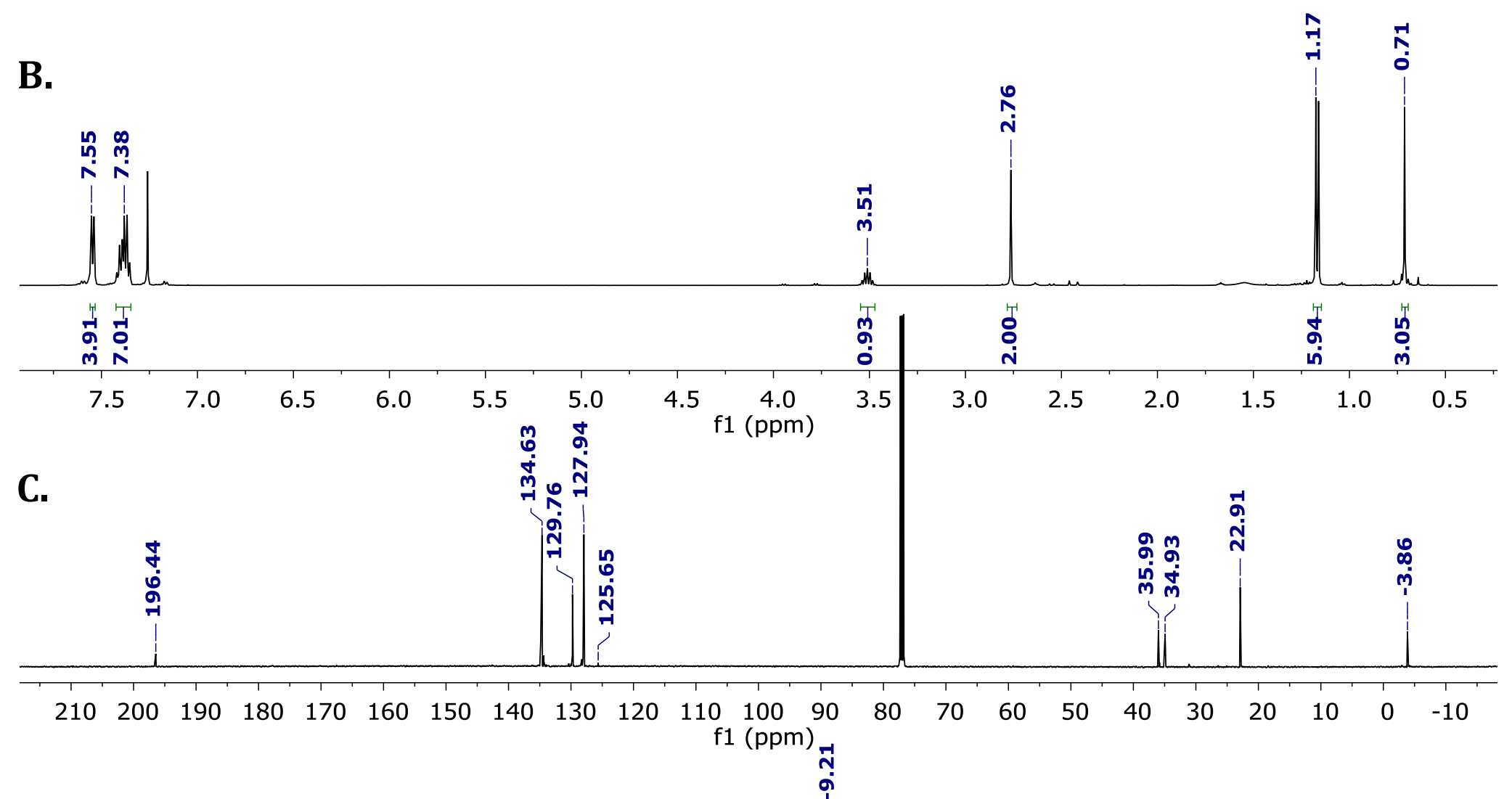

D.
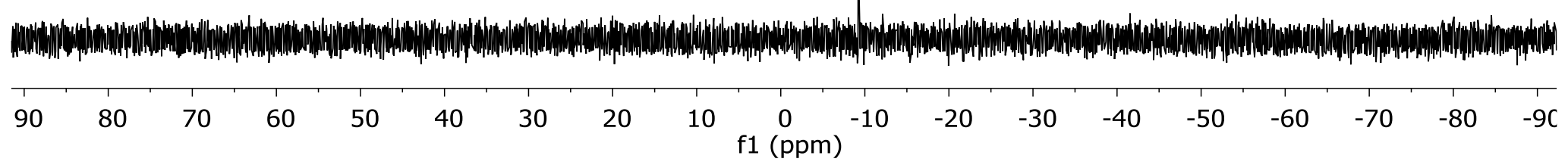
E.

09231907 \#114-126 RT: 0.51-0.56 AV: 13 SB: 12 0.44-0.49 NL: 3.64E7 T: FTMS + p ESI Full ms [50.0000-500.0000]

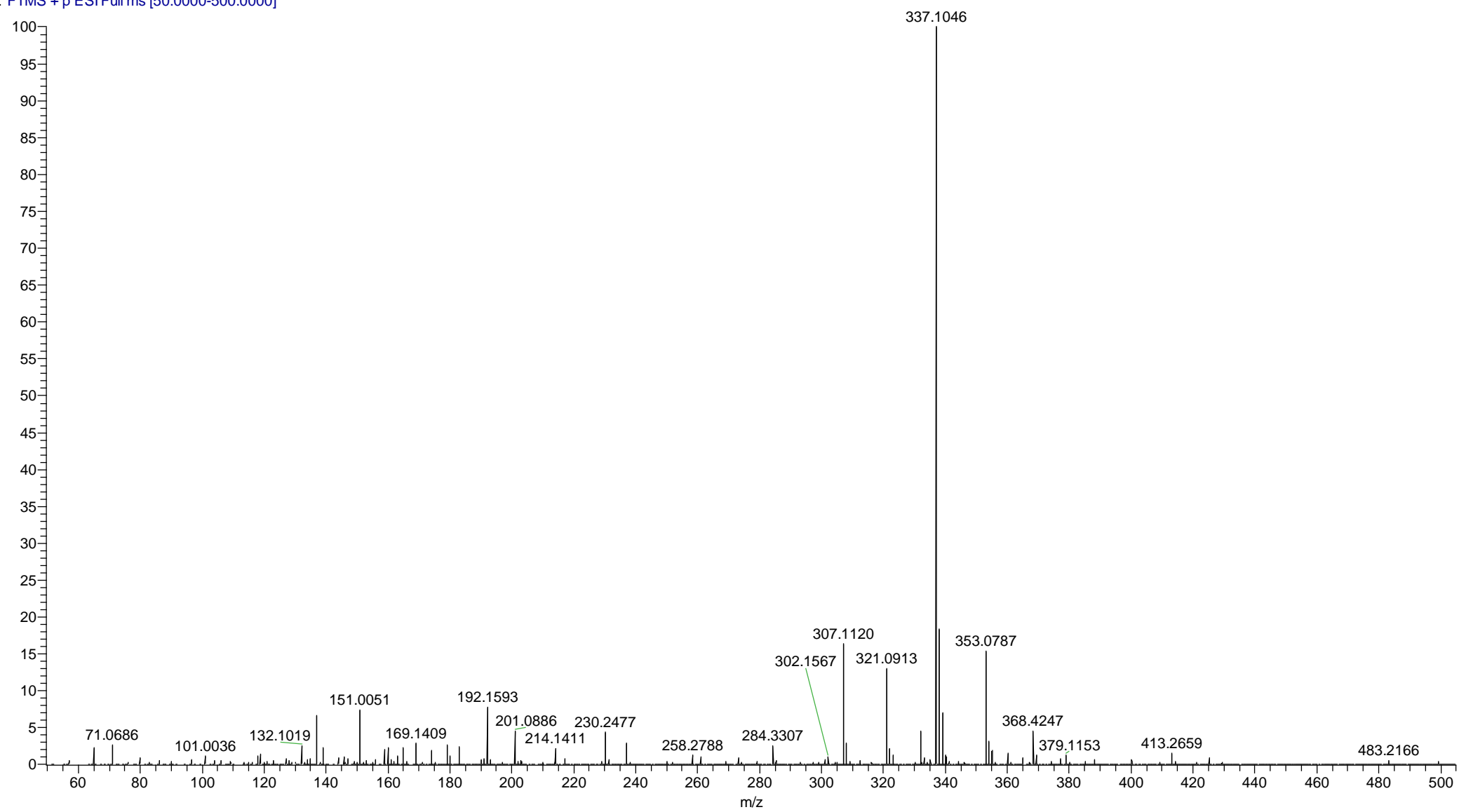


Figure S22. Compound 17 (A) FTIR (B) ${ }^{1} \mathrm{H}$ NMR (C) ${ }^{13} \mathrm{C}$ NMR (D) ${ }^{29}$ Si NMR (E) mass spectroscopy

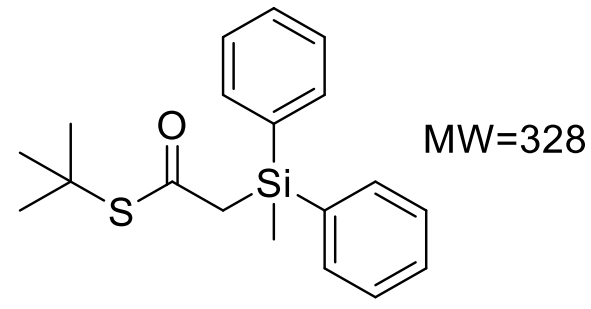

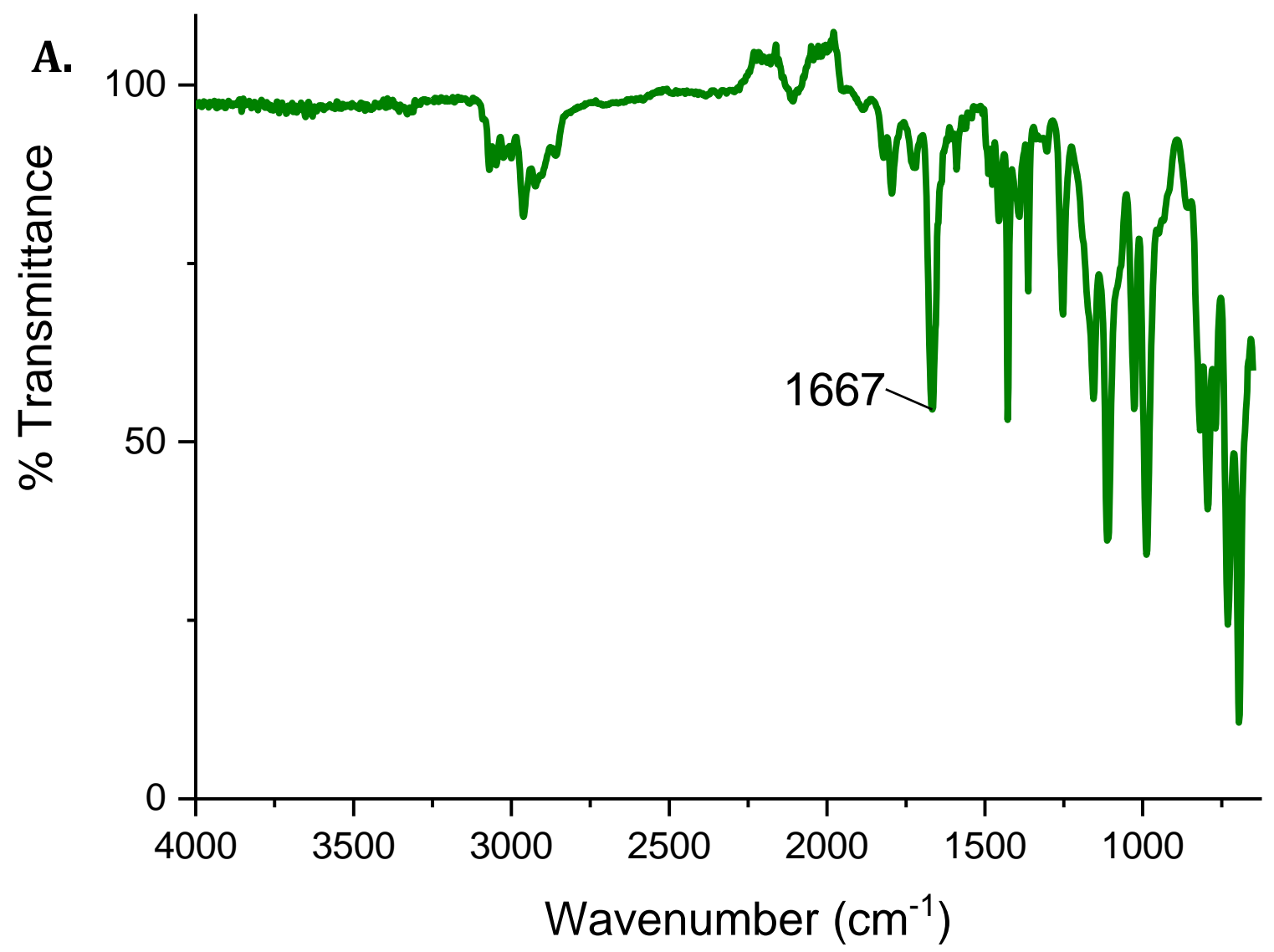


B.

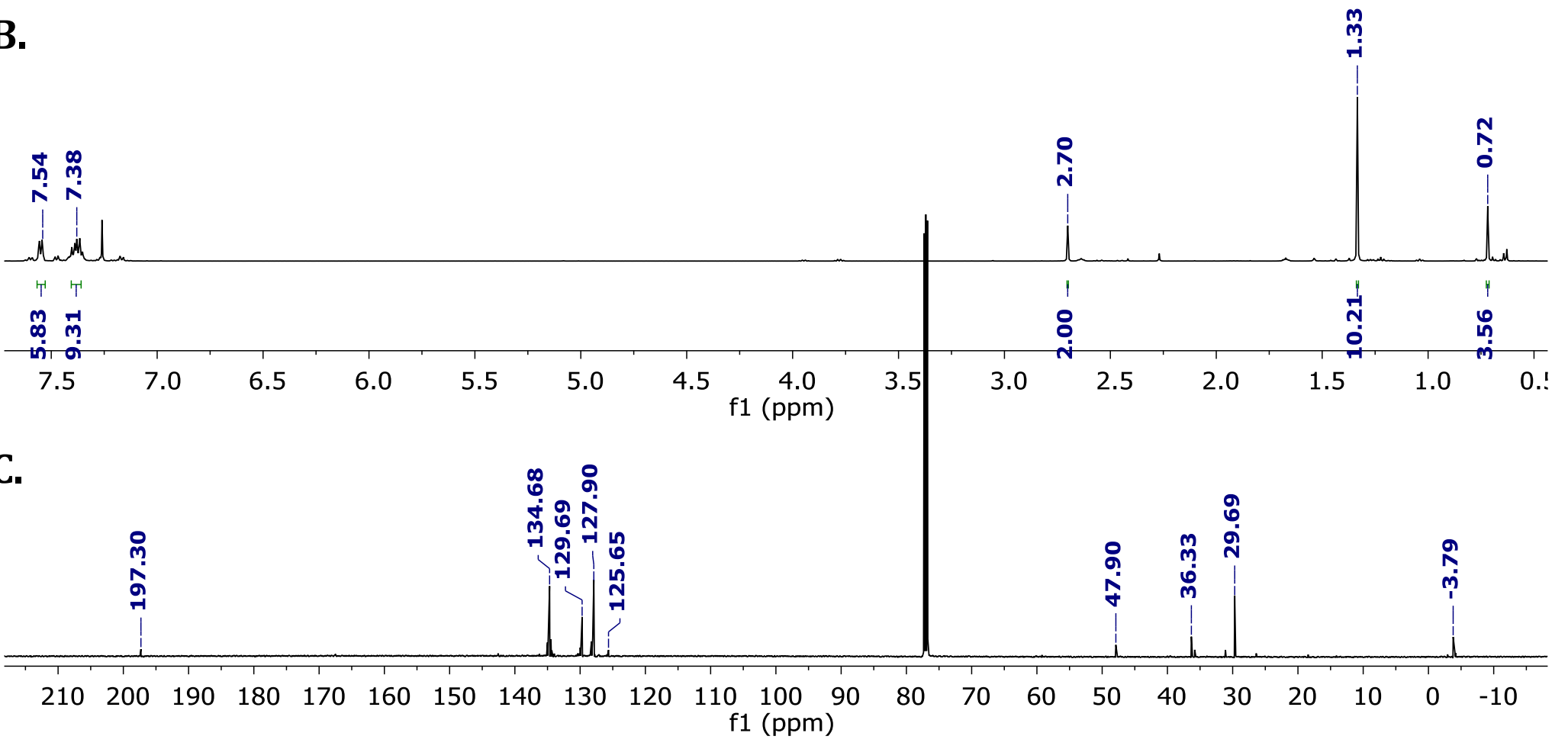

D.

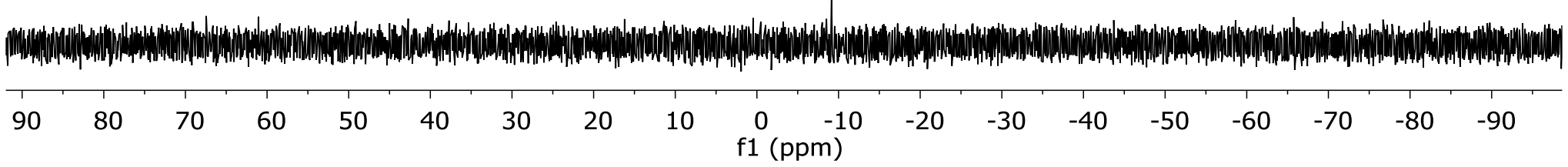


E.

09231908 \#113-118 RT: 0.50-0.53 AV: 6 SB: 13 0.40-0.45 NL: $1.81 \mathrm{E7}$ T: FTMS + p ESI Full ms [50.0000-500.0000]

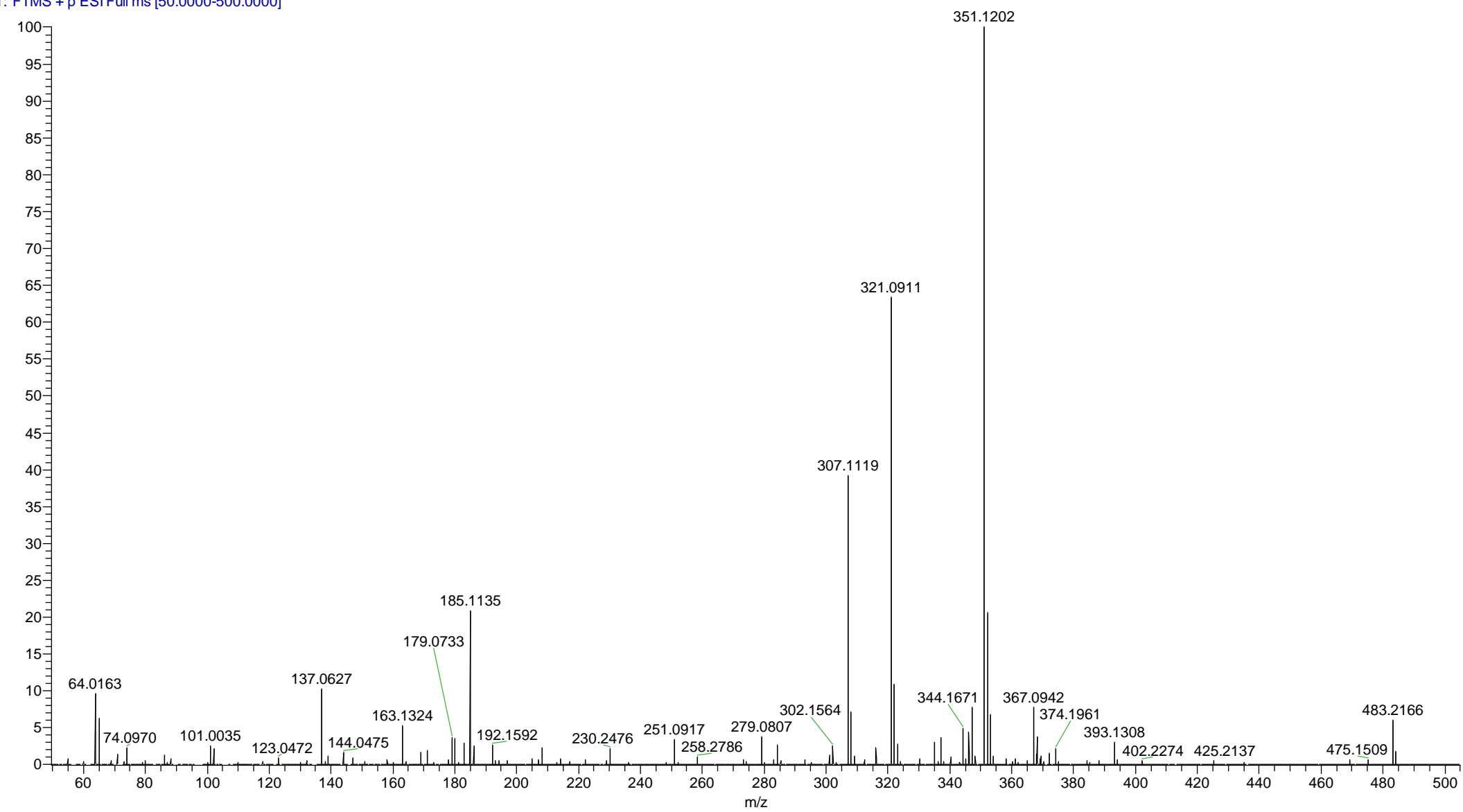


Figure S23. Compound 18 (A) FTIR (B) ${ }^{1} \mathrm{H}$ NMR (C) ${ }^{13} \mathrm{C}$ NMR (D) ${ }^{29}$ Si NMR (E) mass spectroscopy

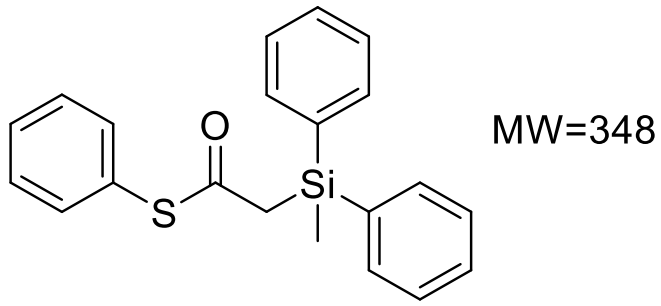

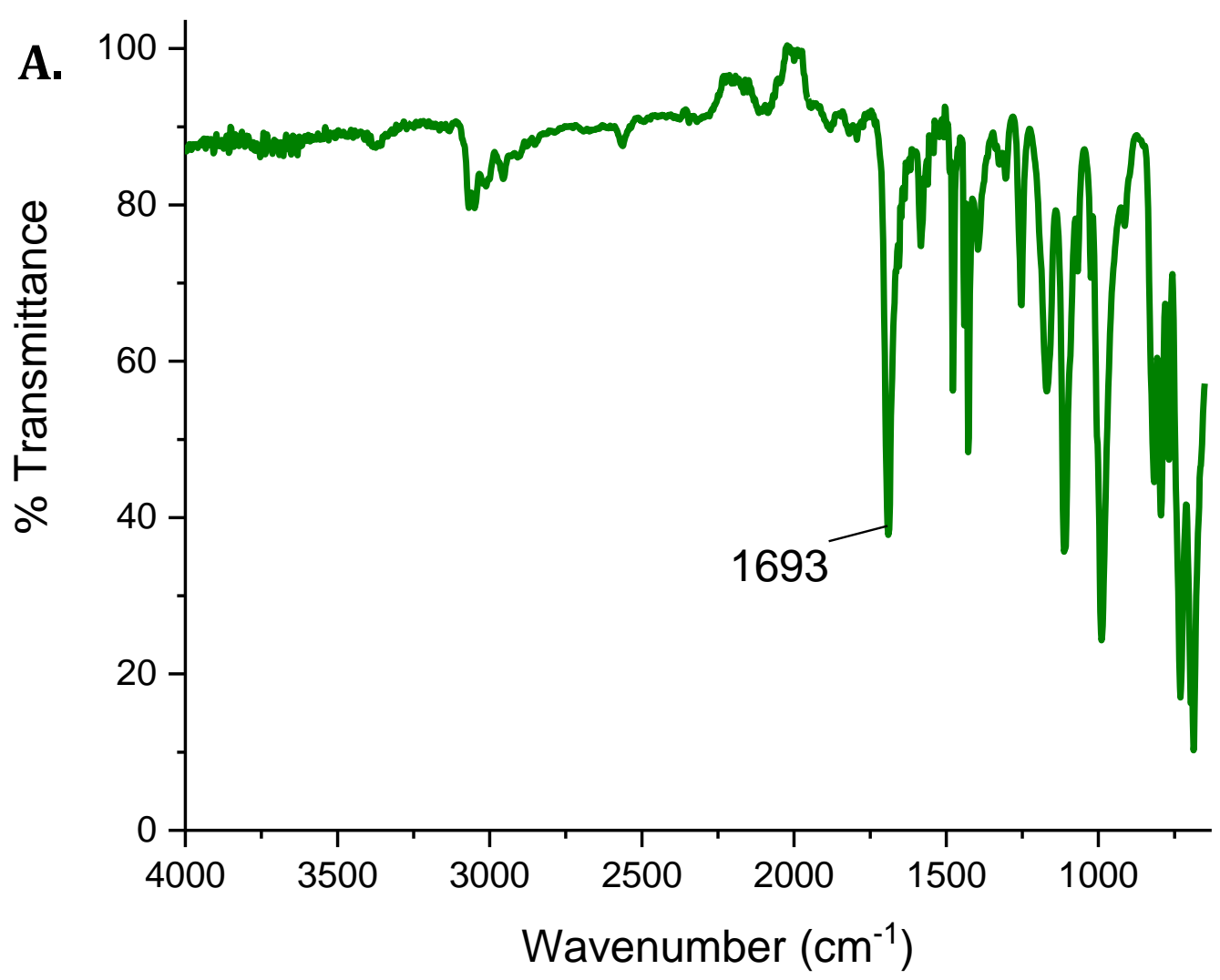




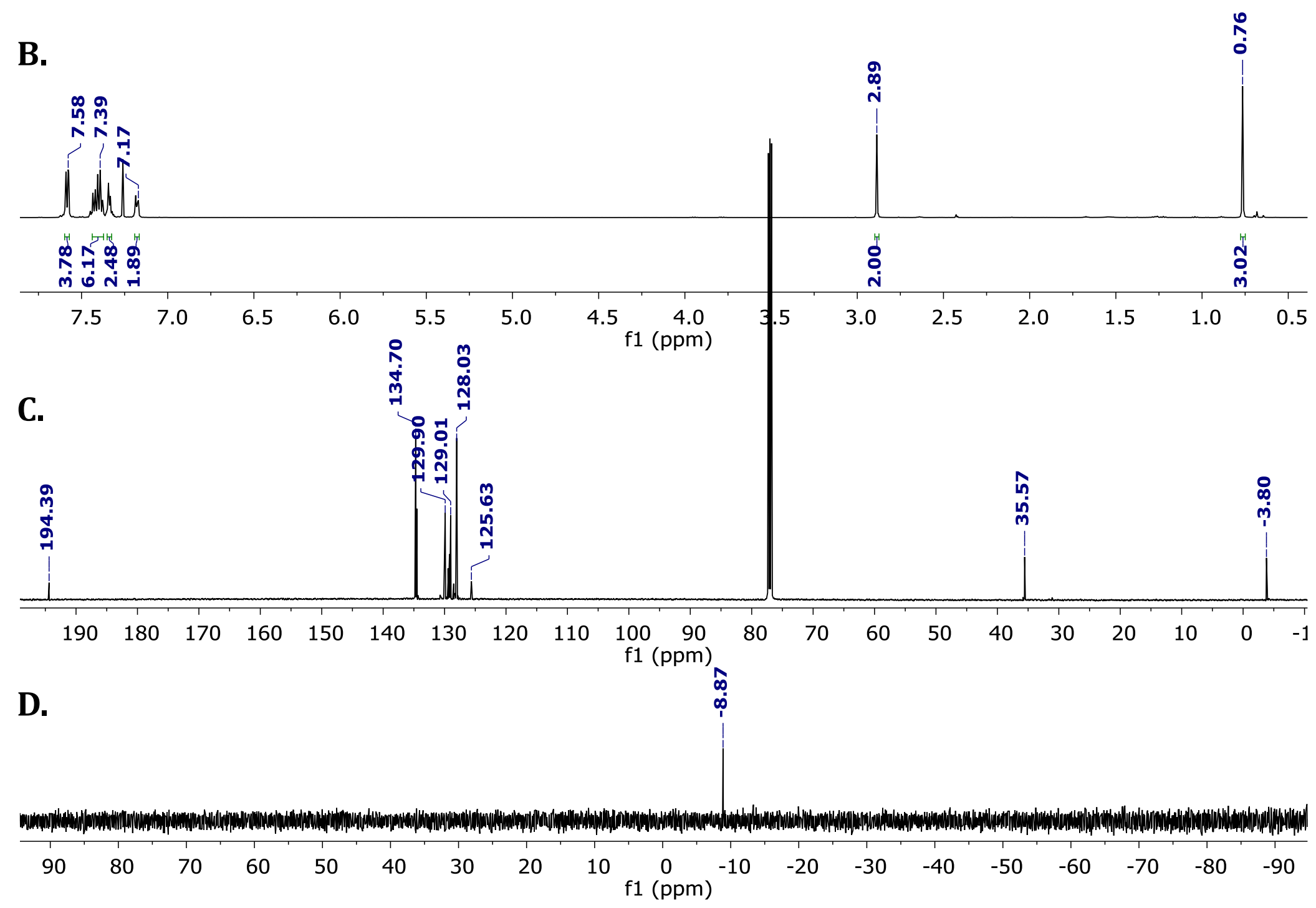


E.

09231909 \#119-126 RT: 0.53-0.56 AV: 8 SB: 12 0.34-0.39 NL: 7.61E7

T: FTMS + p ESI Full ms [50.0000-500.0000]

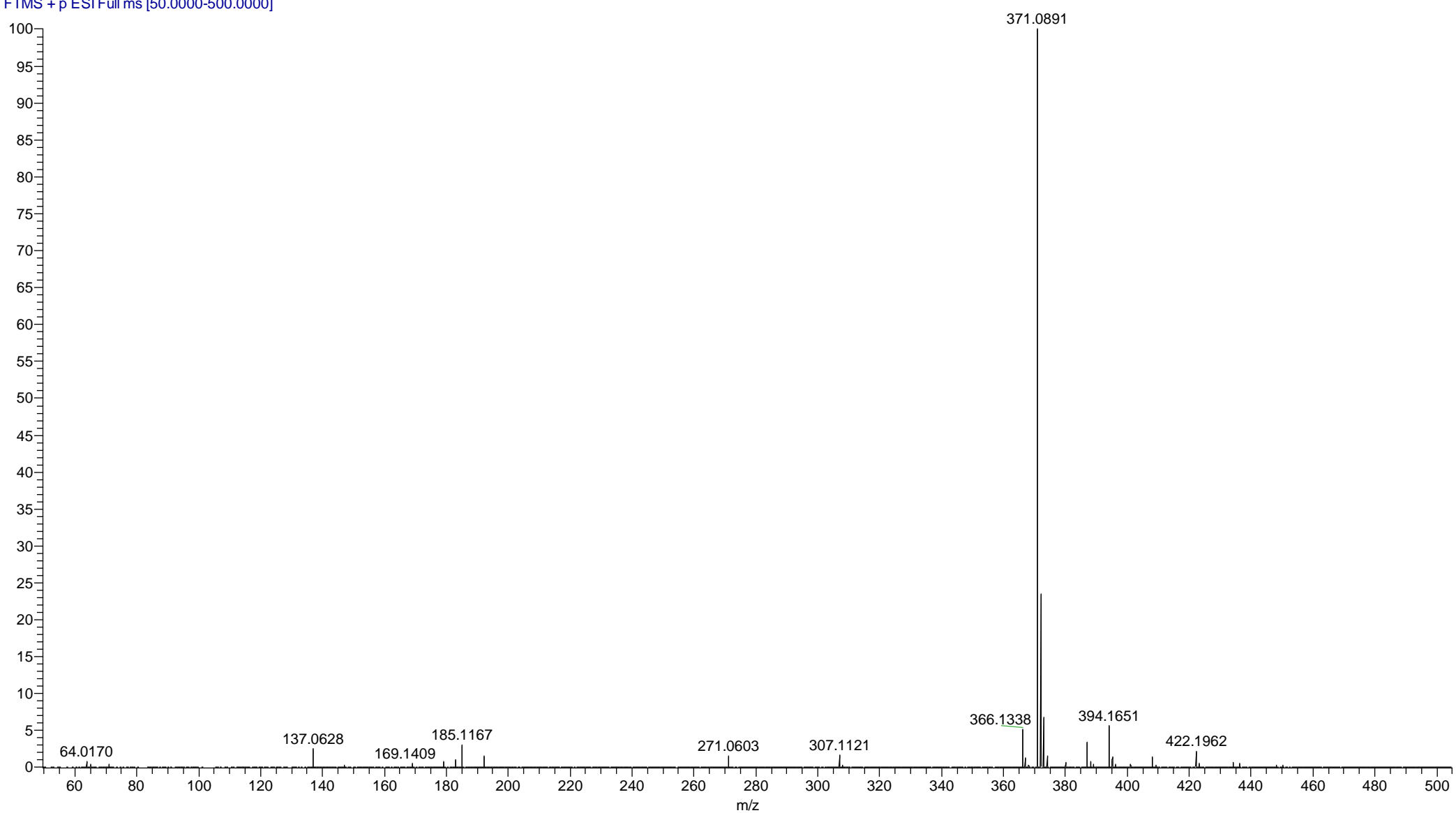


Figure S24. Compound 19 (A) FTIR (B) ${ }^{1} \mathrm{H}$ NMR (C) ${ }^{13} \mathrm{C}$ NMR (D) ${ }^{29}$ Si NMR (E) mass spectroscopy<smiles>COc1ccc(SC(=O)C[Si](C)(c2ccccc2)c2ccccc2)cc1</smiles>

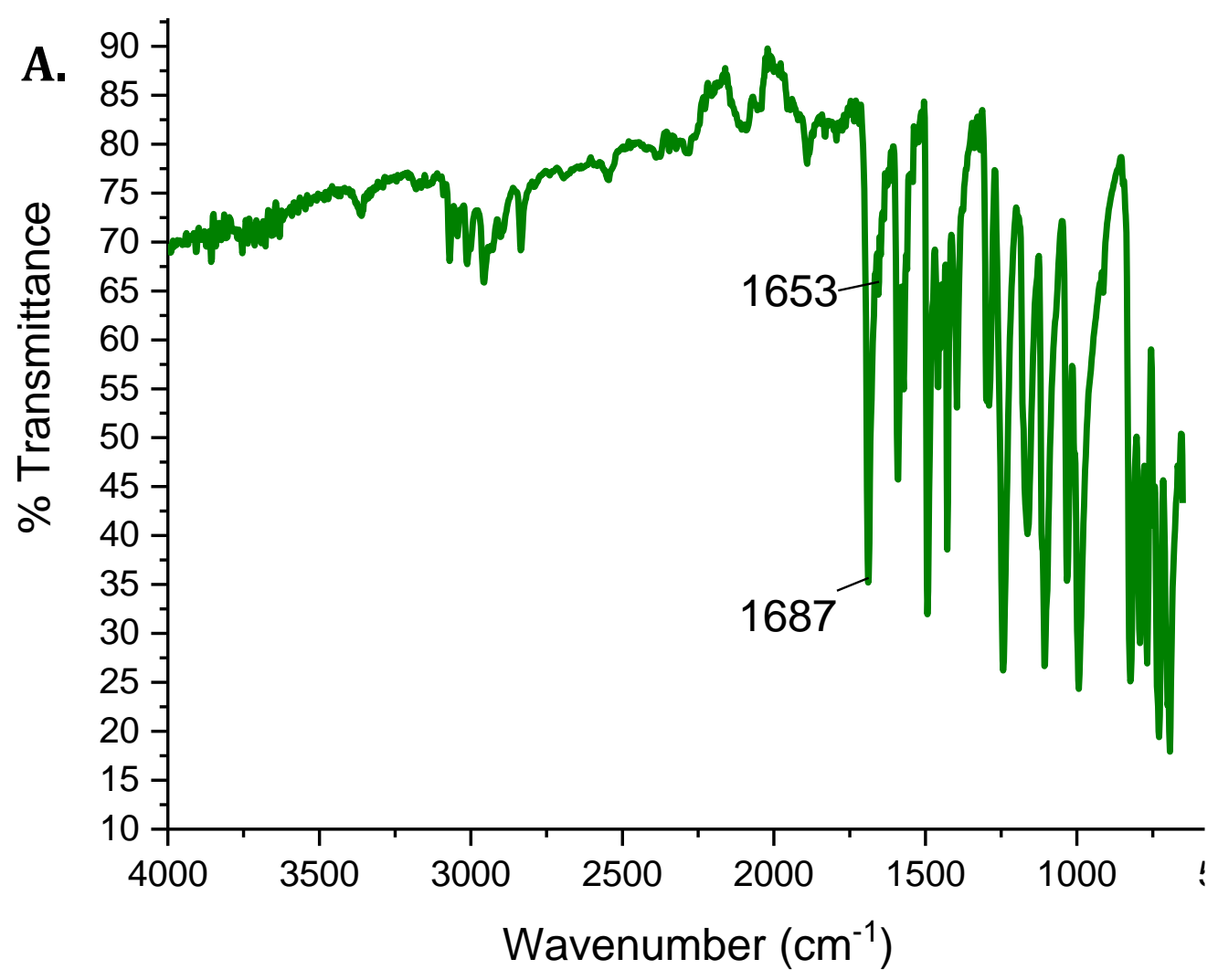




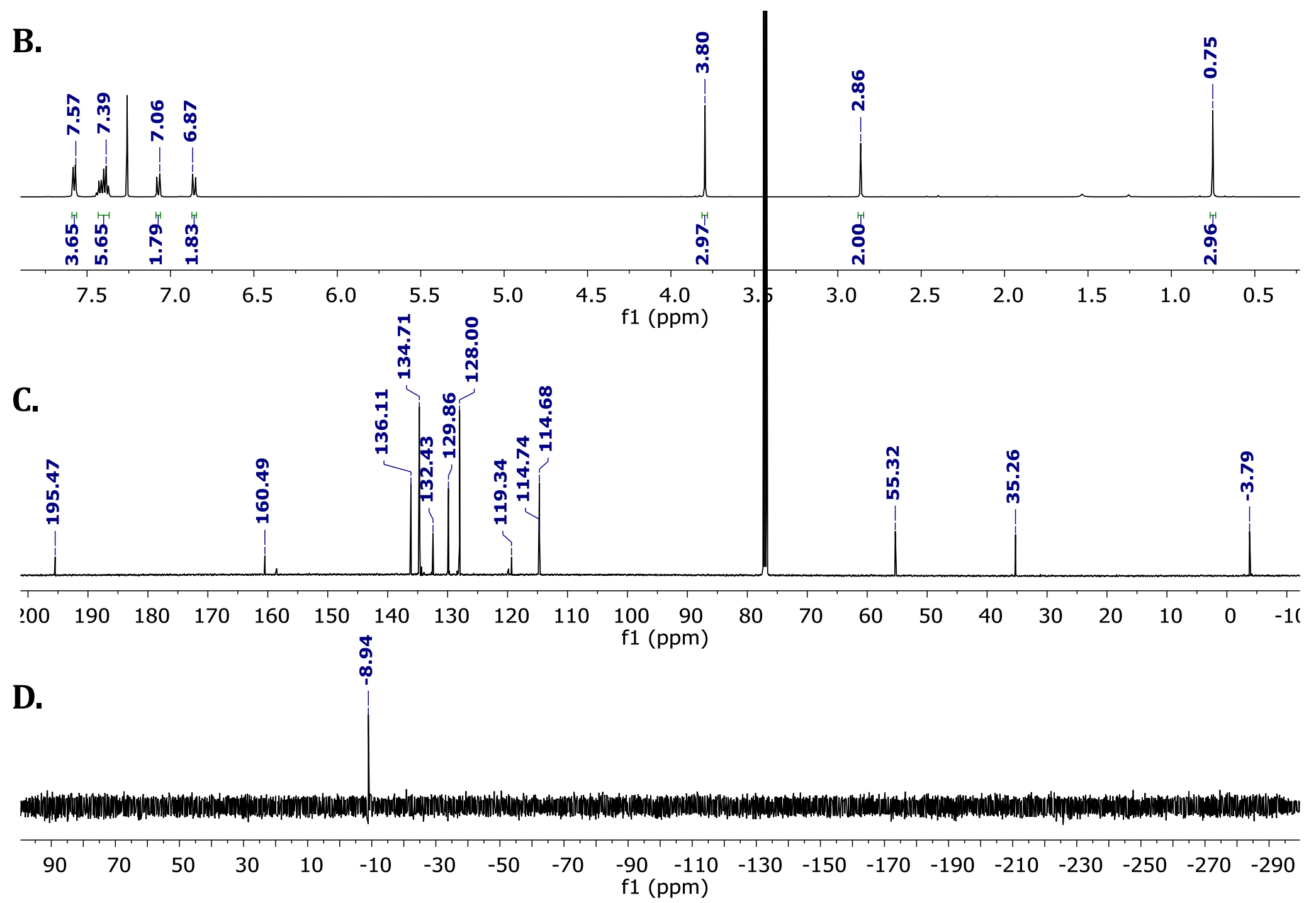


E.

09231910 \#116-121 RT: 0.52-0.54 AV: 6 SB: 19 0.35-0.43 NL: 3.75E7 T: FTMS + p ESI Full ms [50.0000-500.0000]

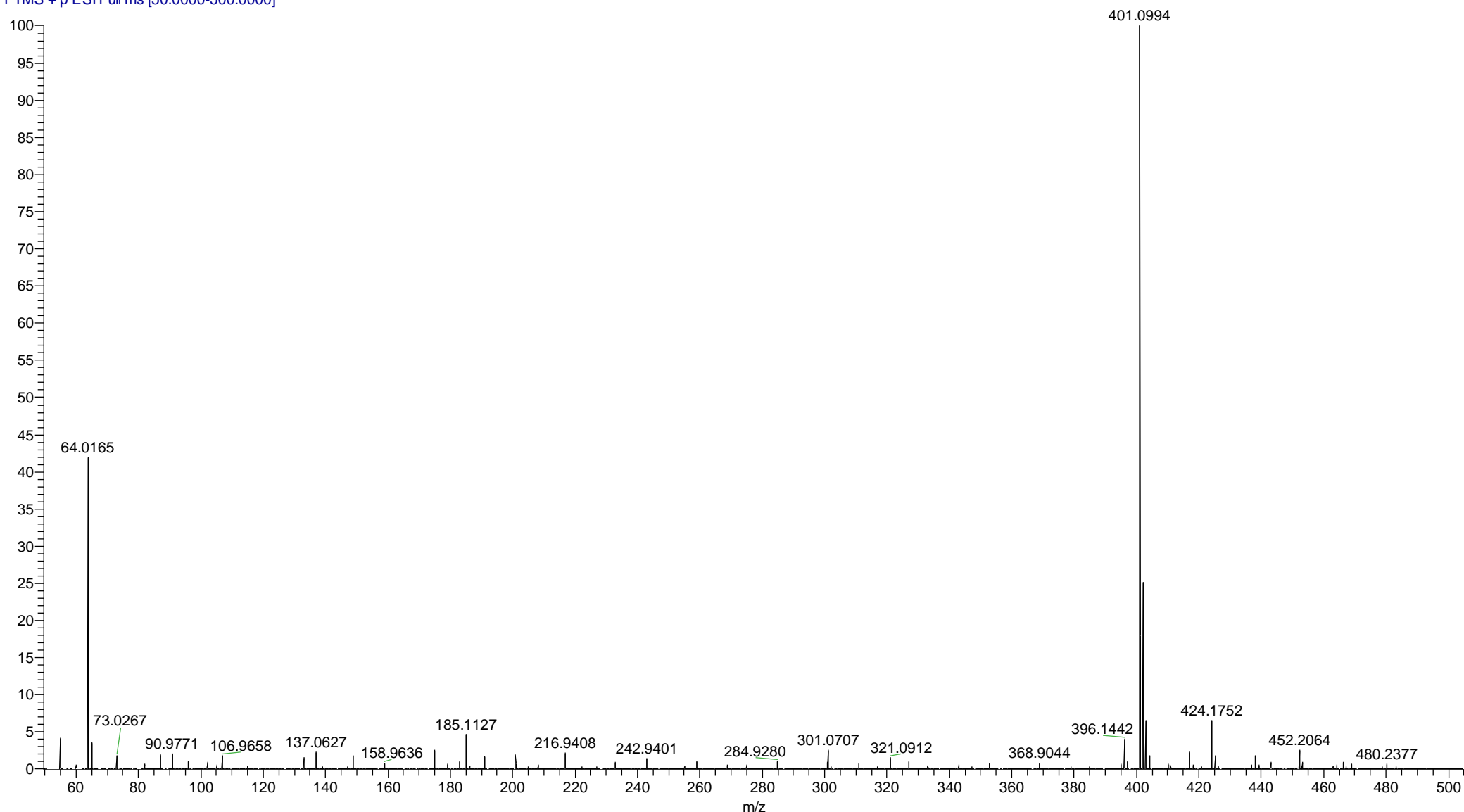


Figure S25. Compound 20 (A) FTIR (B) ${ }^{1} \mathrm{H}$ NMR (C) ${ }^{13} \mathrm{C}$ NMR (D) ${ }^{29}$ Si NMR (E) mass spectroscopy

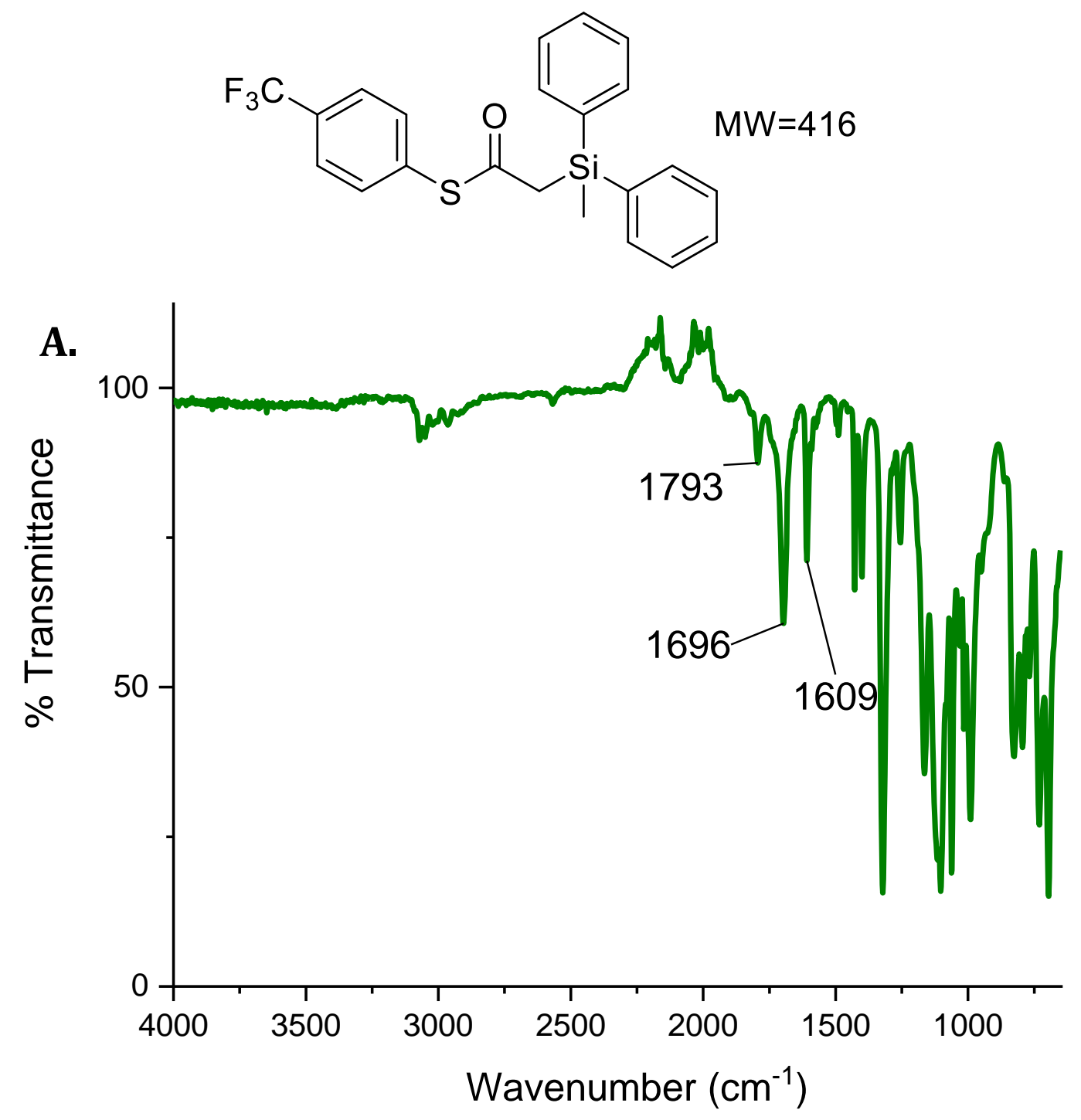




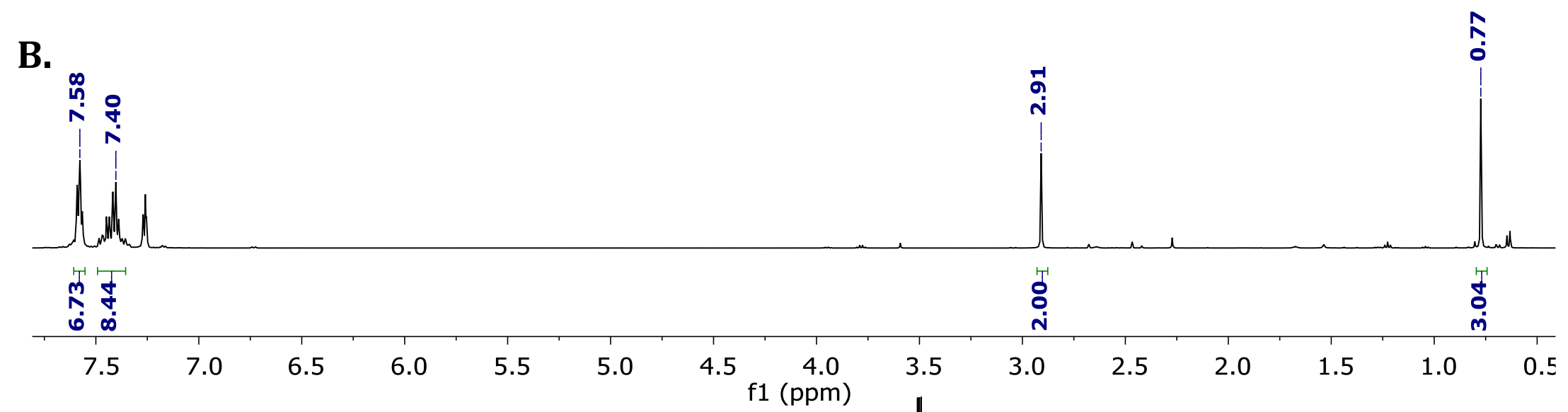

c.

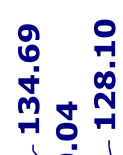

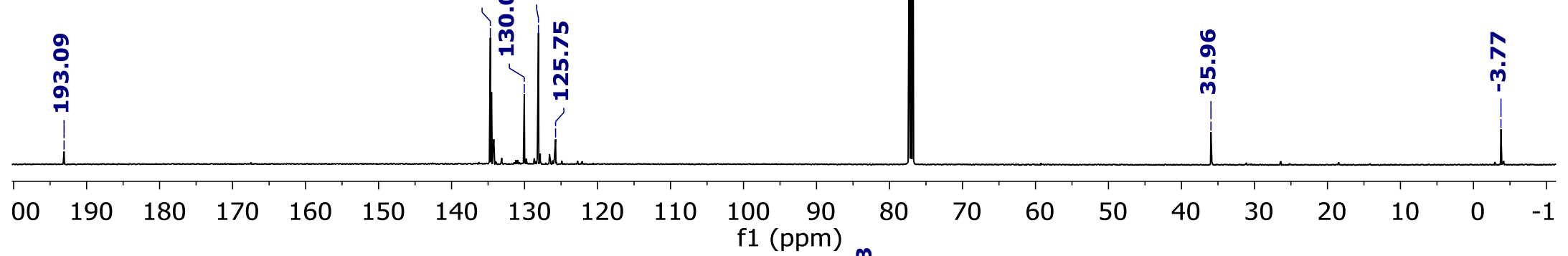

D.
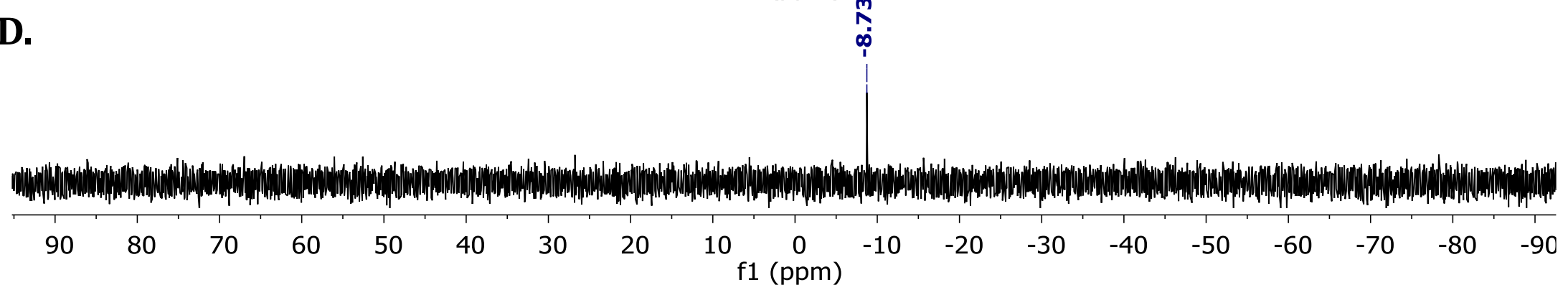
E.

09231911 \#109-119 RT: 0.49-0.53 AV: 11 SB: 11 0.43-0.48 NL: 1.98E7 T: FTMS + p ESI Full ms [50.0000-600.0000]

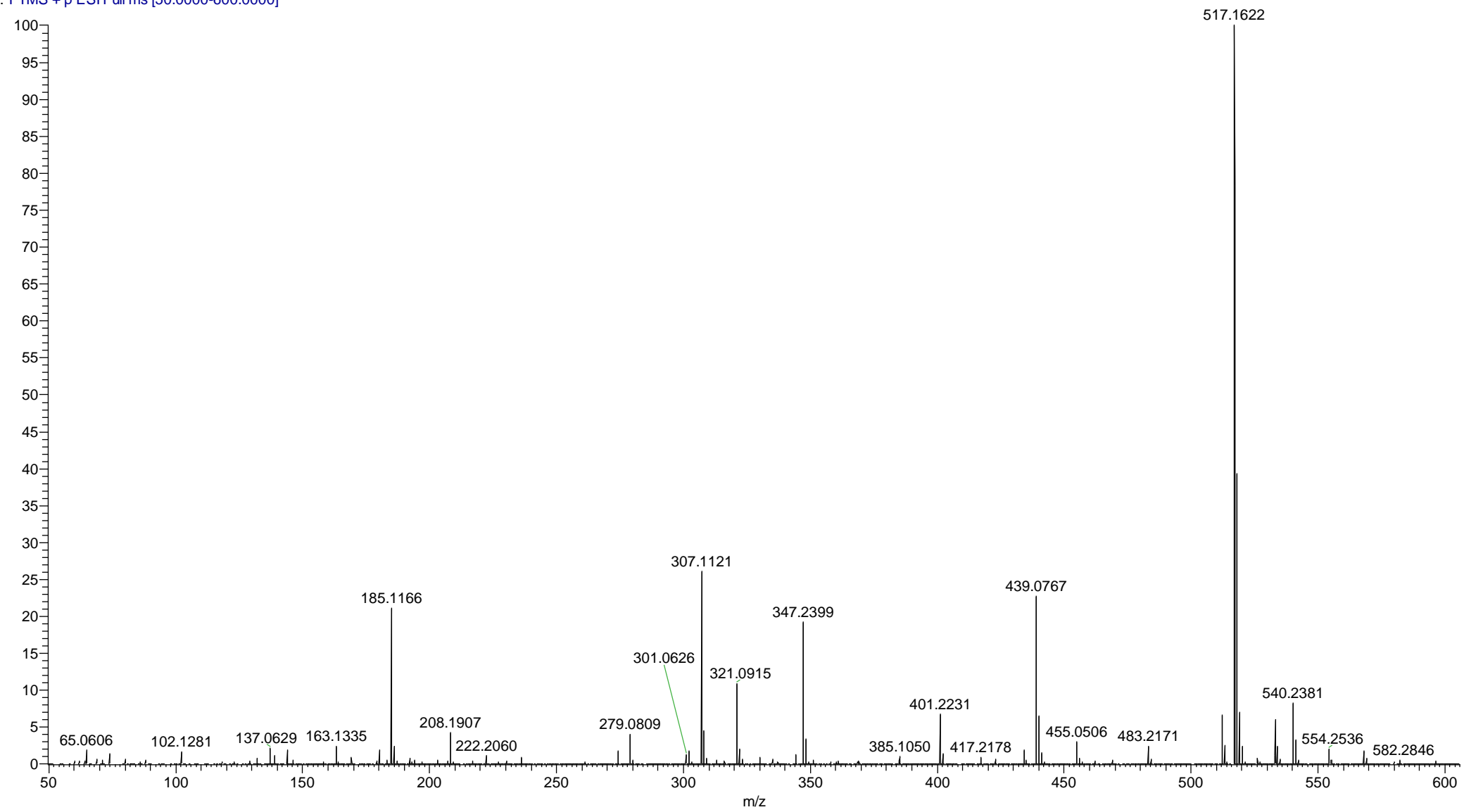


Figure S26. Compound 21 (A) FTIR (B) ${ }^{1} \mathrm{H}$ NMR (C) ${ }^{13} \mathrm{C}$ NMR (D) ${ }^{29}$ Si NMR (E) mass spectroscopy

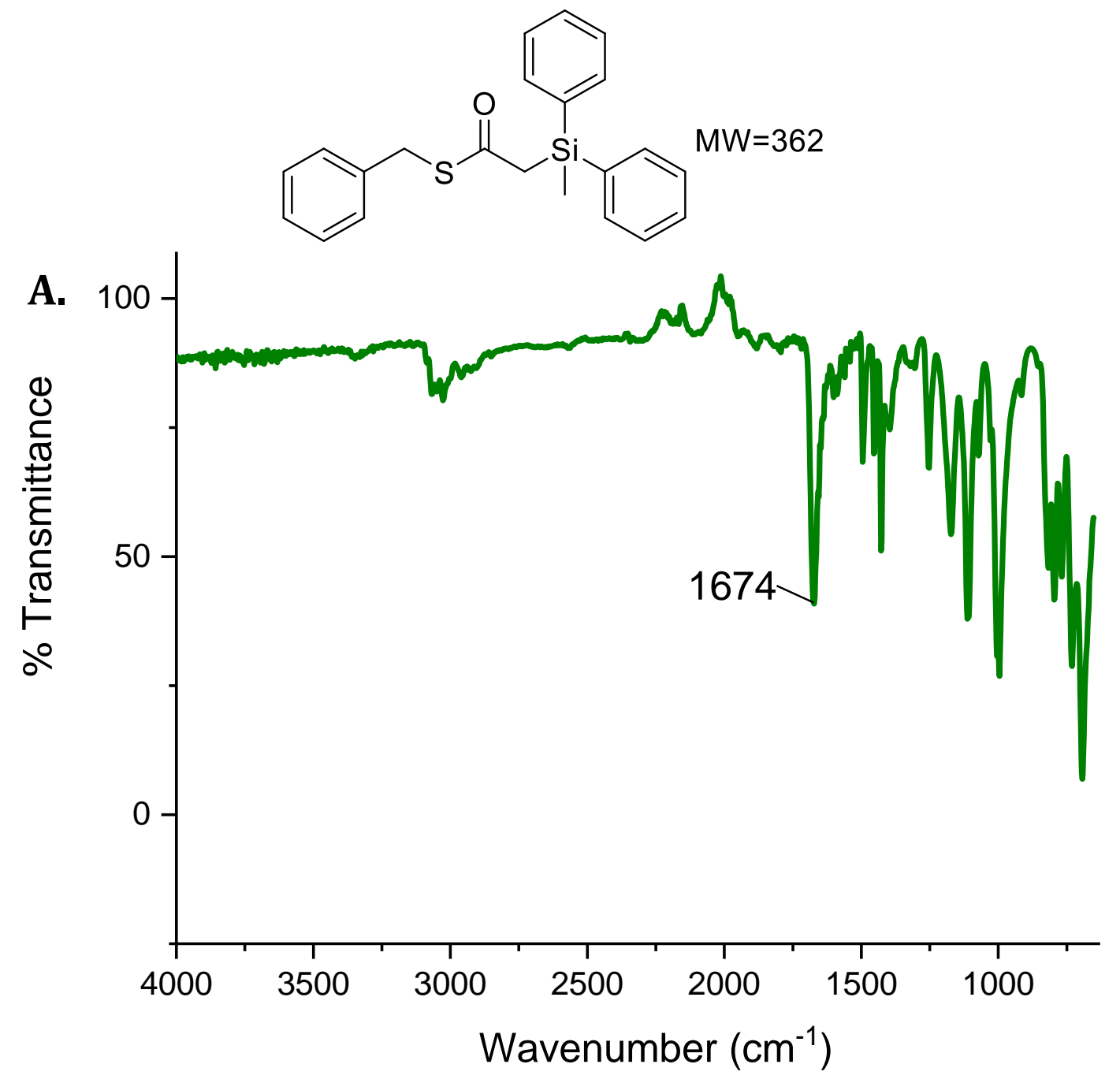




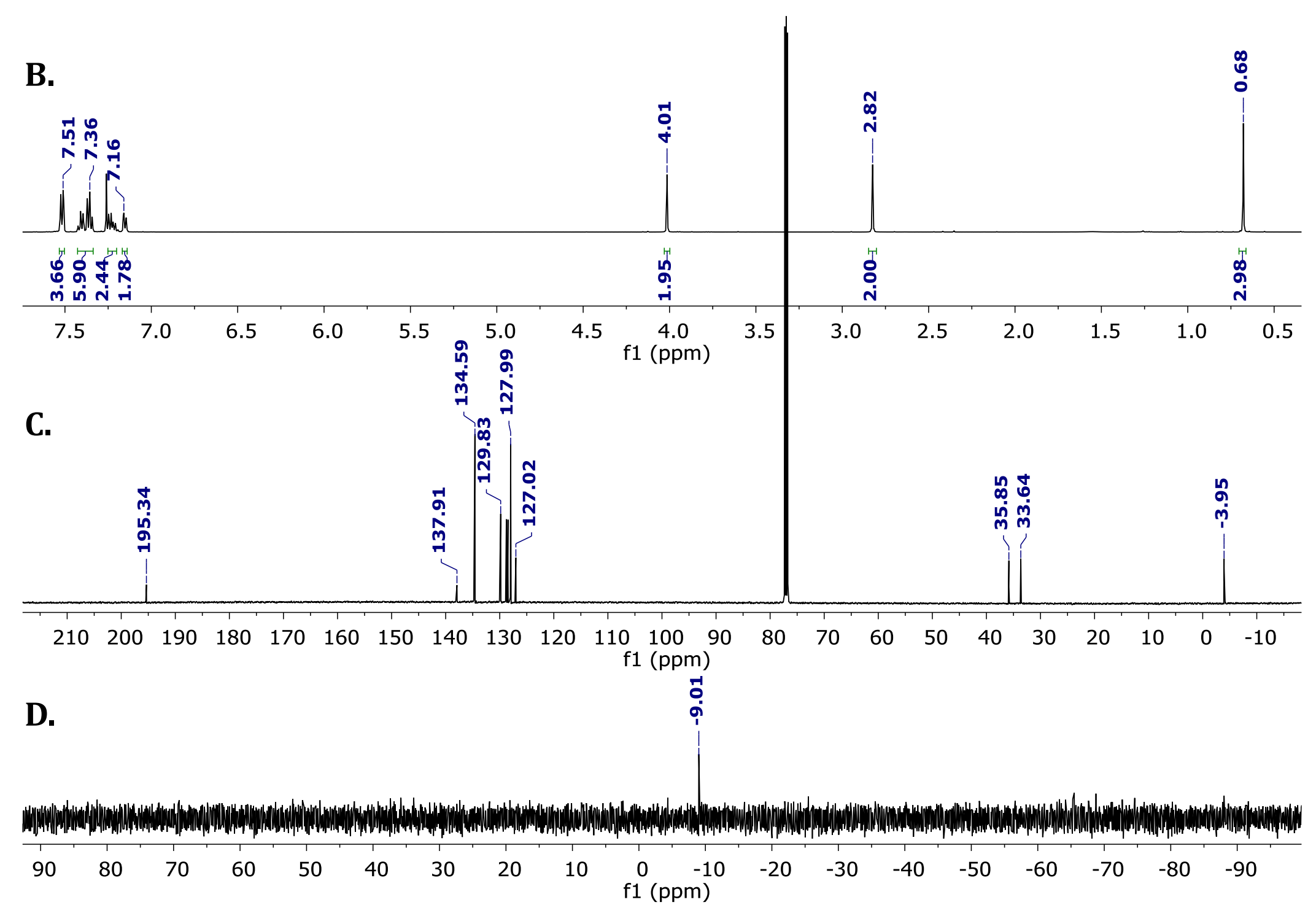


E.

09231912 \#118-126 RT: 0.53-0.56 AV: 9 SB: 17 0.40-0.47 NL: 2.69E7 T: FTMS + p ESI Full ms [50.0000-500.0000]

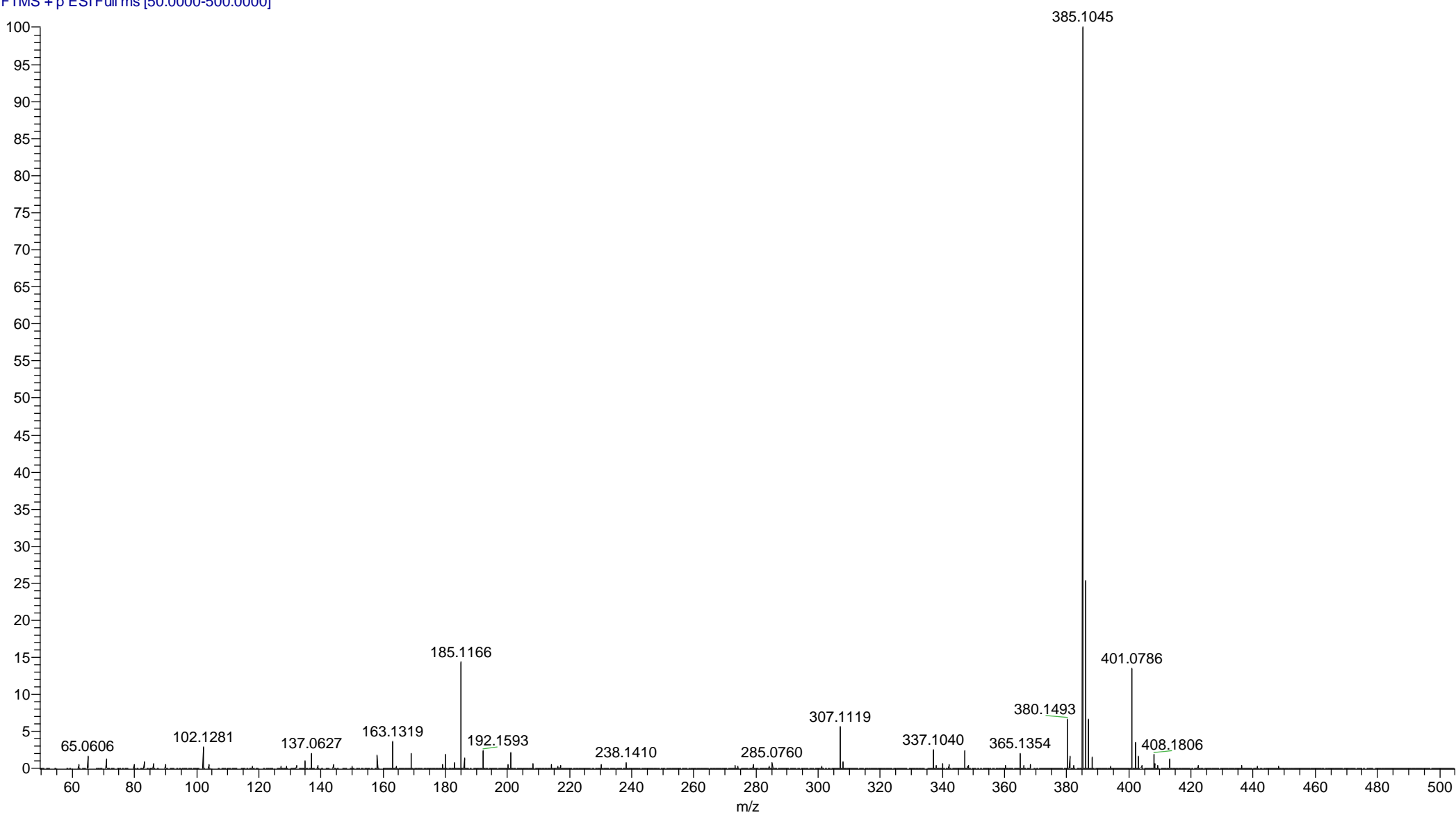


Figure S27. Compound TES 1 (A) FTIR (B) ${ }^{1} \mathrm{H}$ NMR (C) ${ }^{13} \mathrm{C}$ NMR (D) ${ }^{29} \mathrm{Si}$ NMR (E) mass spectroscopy<smiles>CC[Si](CC)(CC)CC(=O)Oc1ccc(OC)cc1</smiles>

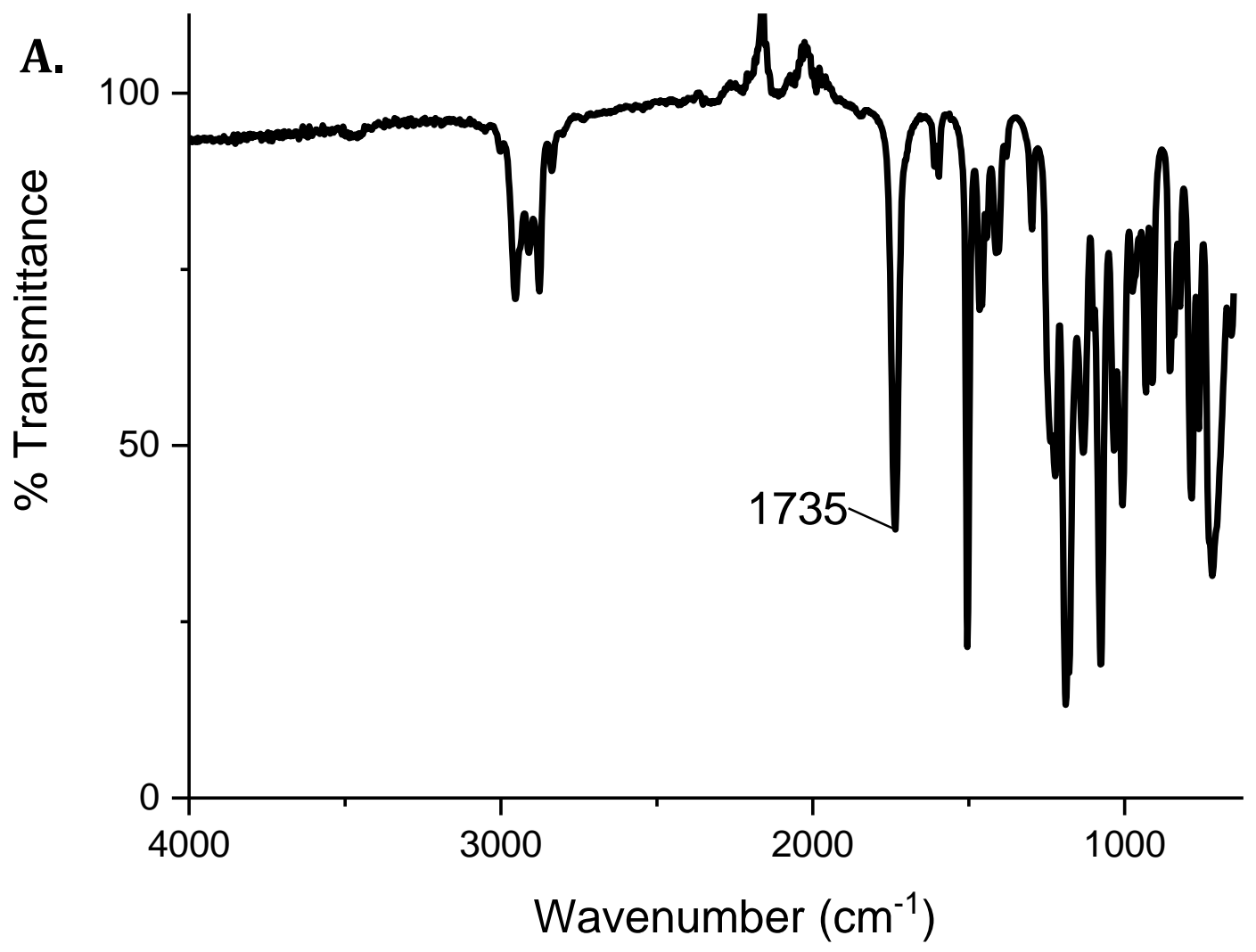




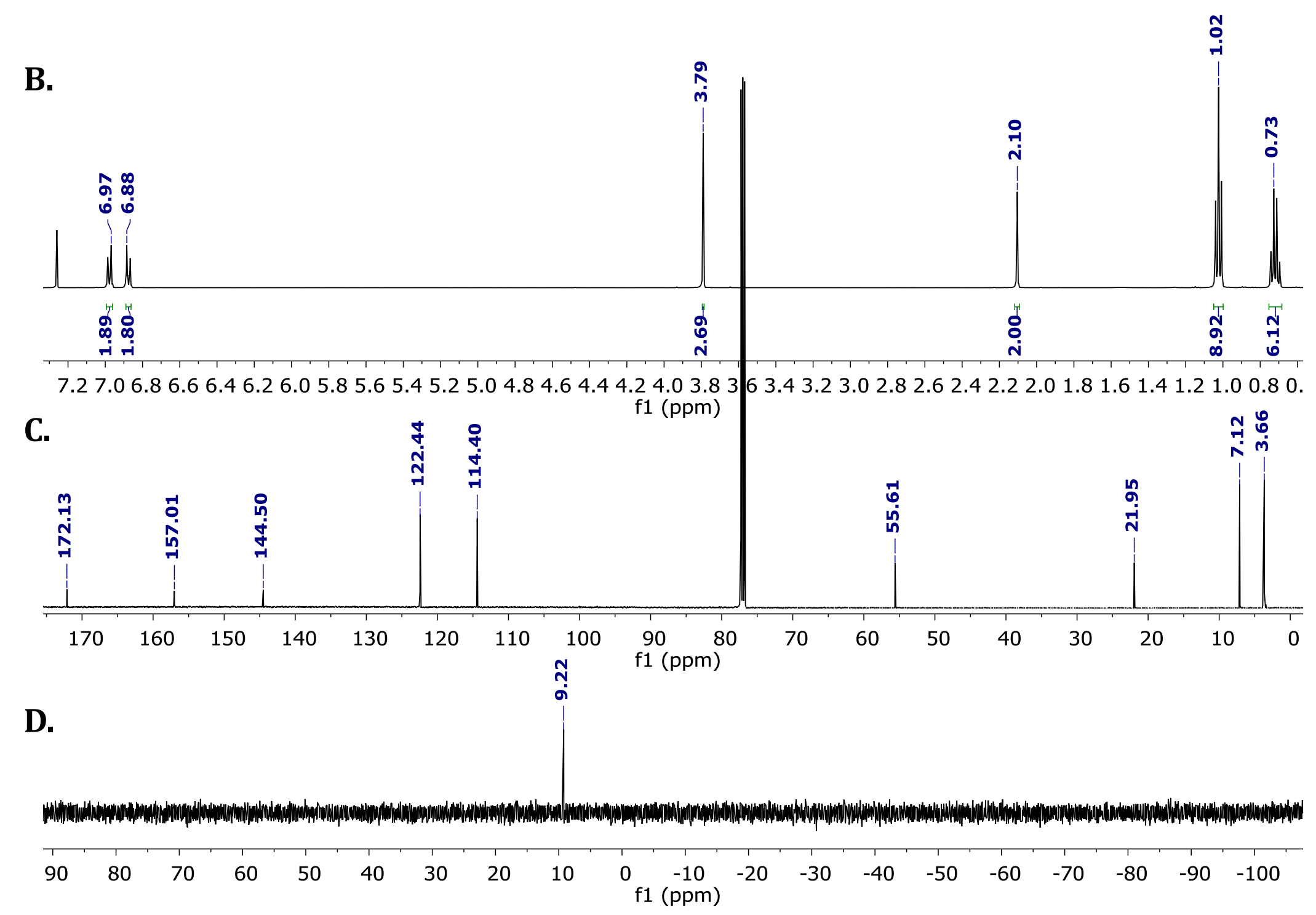


E.

09241909 \#101-108 RT: 0.45-0.48 AV: 8 SB: 16 0.33-0.39 NL: 2.80E8 T: FTMS + p ESI Full ms [50.0000-500.0000]

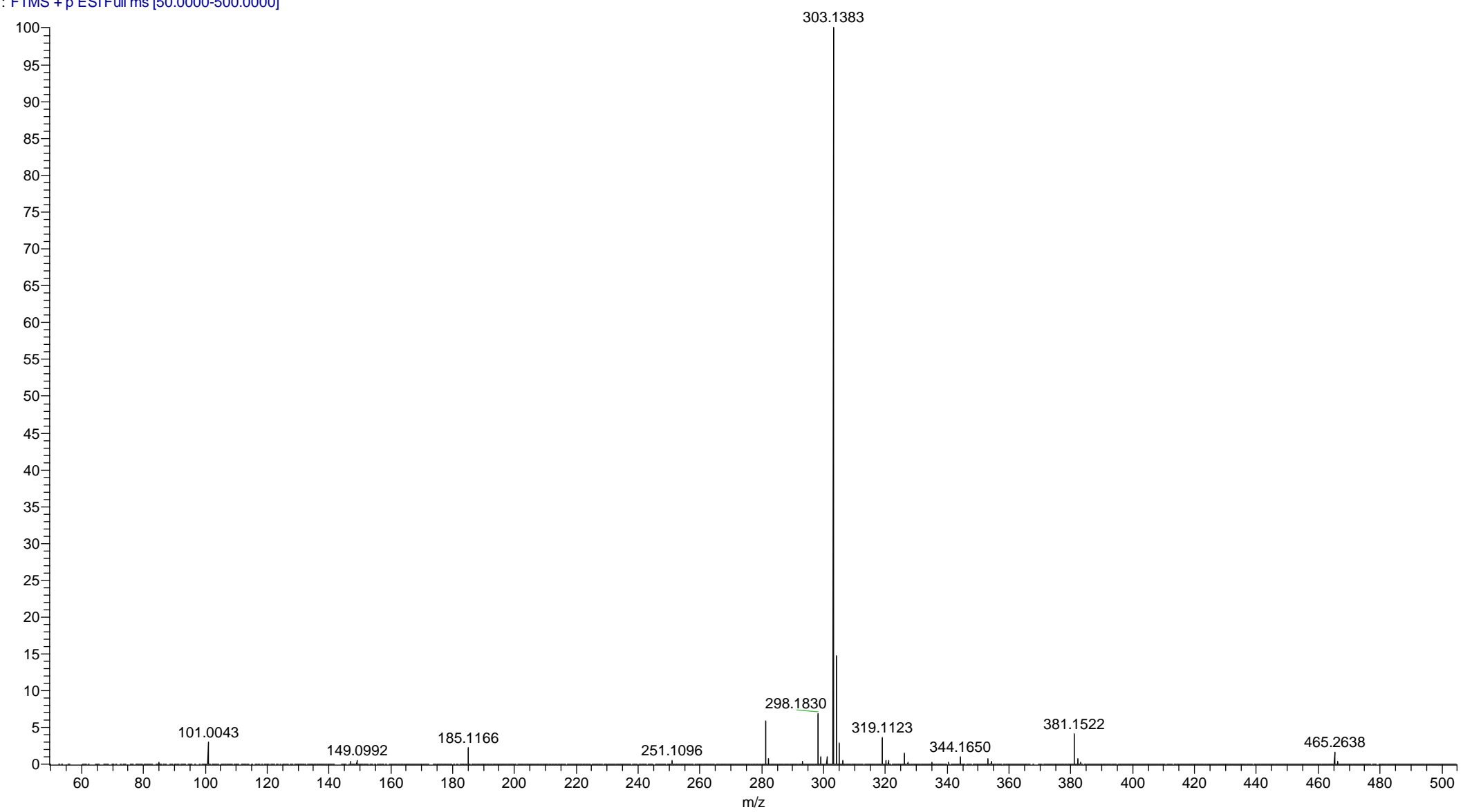


Figure S28. Compound TES 2 (A) FTIR (B) ${ }^{1} \mathrm{H}$ NMR (C) ${ }^{13} \mathrm{C}$ NMR (D) ${ }^{29}$ Si NMR (E) mass spectroscopy<smiles>CC[Si](CC)(CC)CC(=O)Sc1ccc(OC)cc1</smiles>

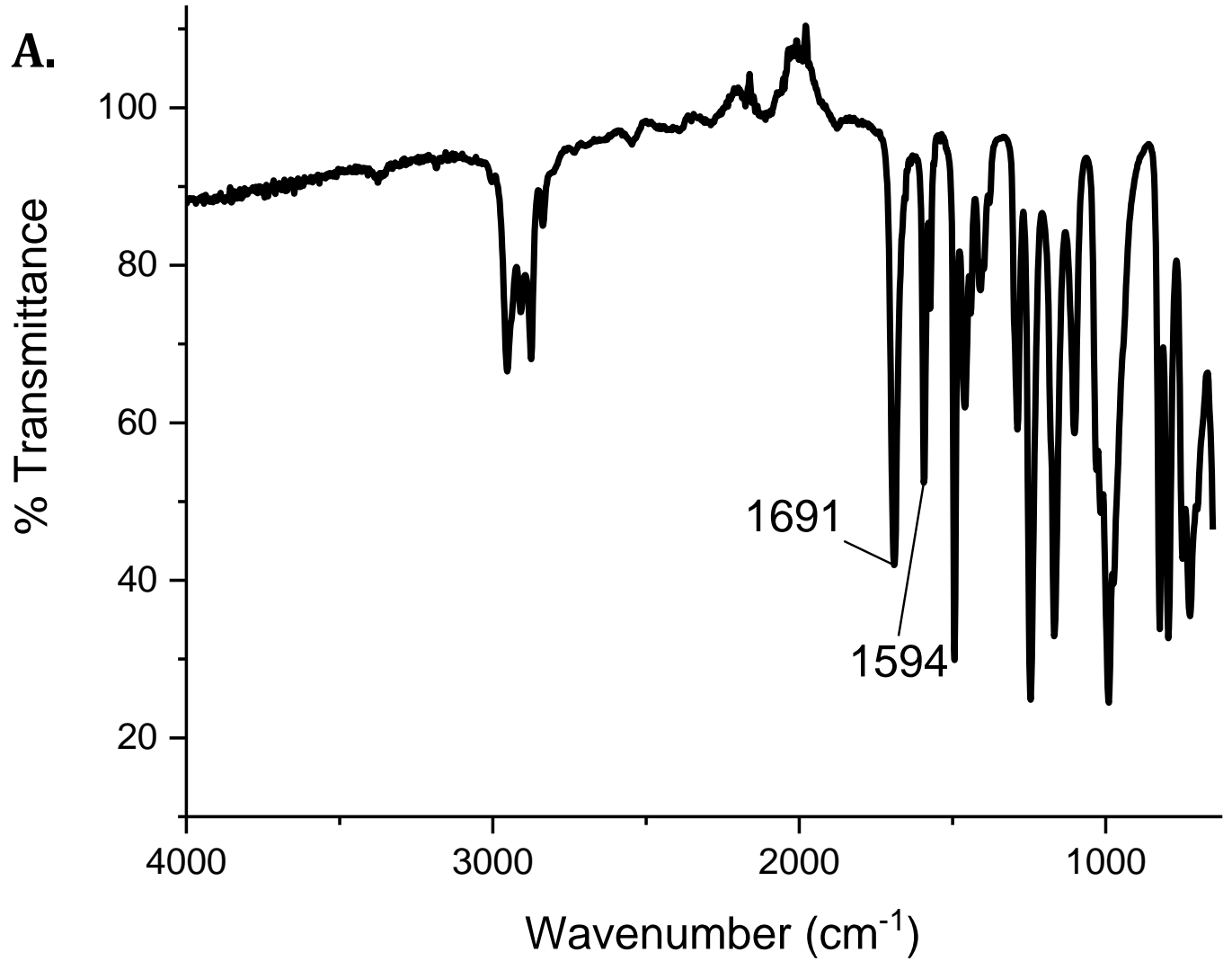


B.

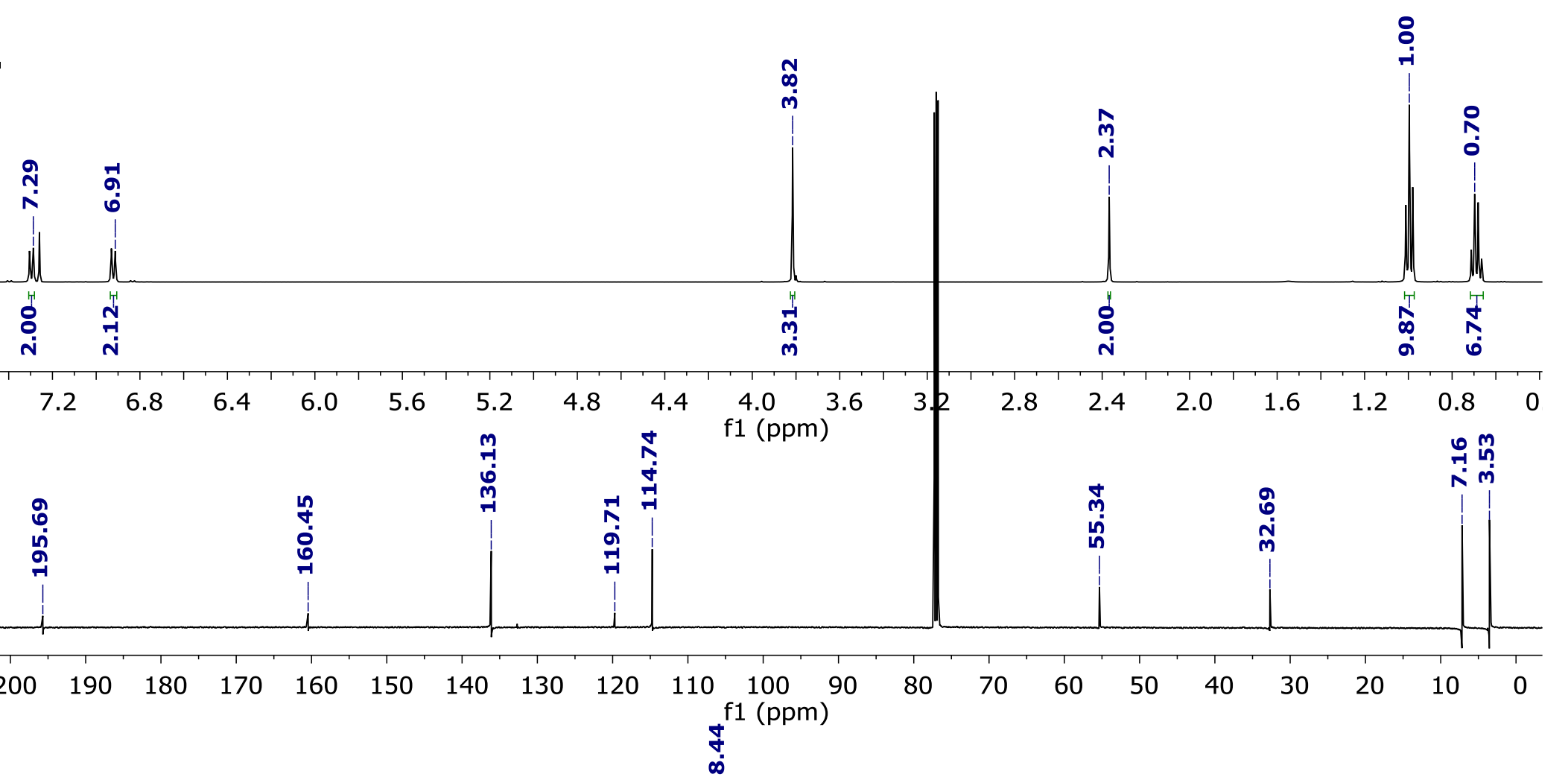

D.

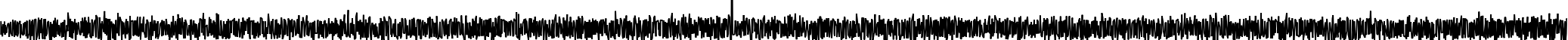

$\begin{array}{llllllllllllllllll}90 & 80 & 70 & 60 & 50 & 40 & 30 & 20 & 10 \underset{f 1(\mathrm{ppm})}{0} & -10 & -20 & -30 & -40 & -50 & -60 & -70 & -80 & -90\end{array}$


E.

09241910 \#127-163 RT: 0.57-0.73 AV: 37 SB: 20 0.07-0.16 NL: 1.72E8 T: FTMS + p ESI Full ms [50.0000-500.0000]

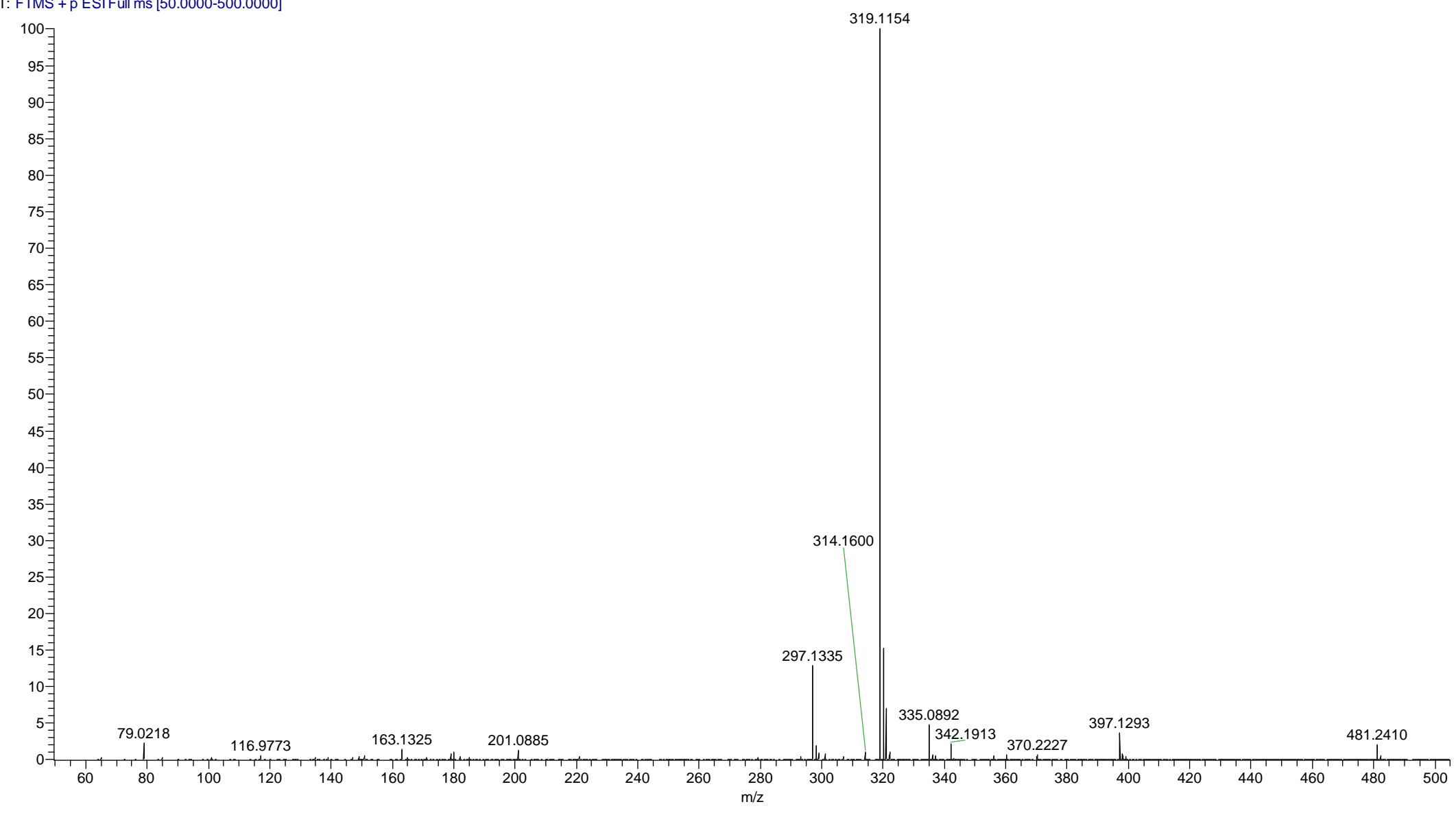


Figure S29. Compound TES 3 (A) FTIR (B) ${ }^{1} \mathrm{H}$ NMR (C) ${ }^{13} \mathrm{C}$ NMR (D) ${ }^{29} \mathrm{Si}$ NMR (E) mass spectroscopy<smiles>CC[Si](CC)(CC)CC(=O)Nc1ccccc1</smiles>

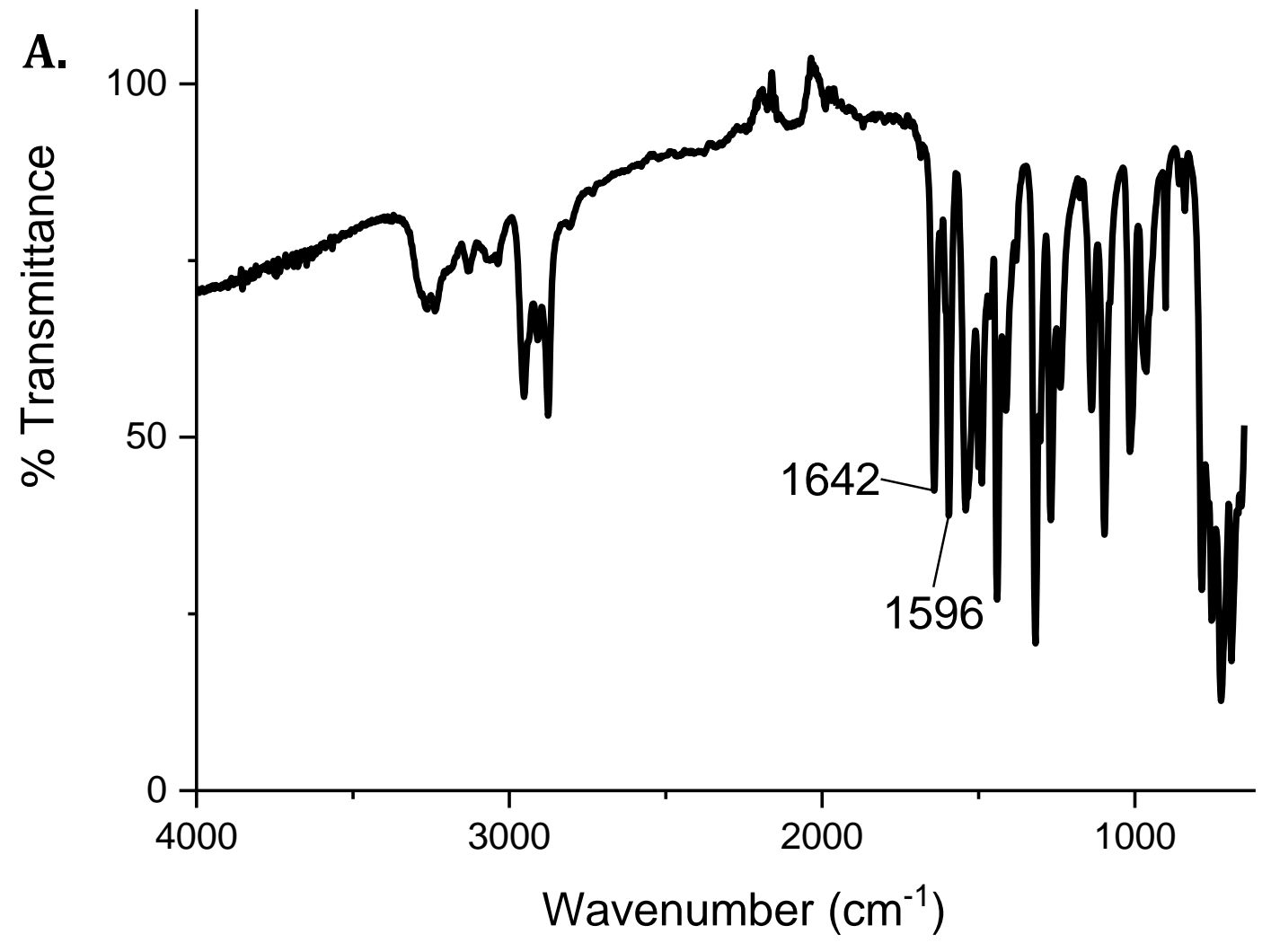




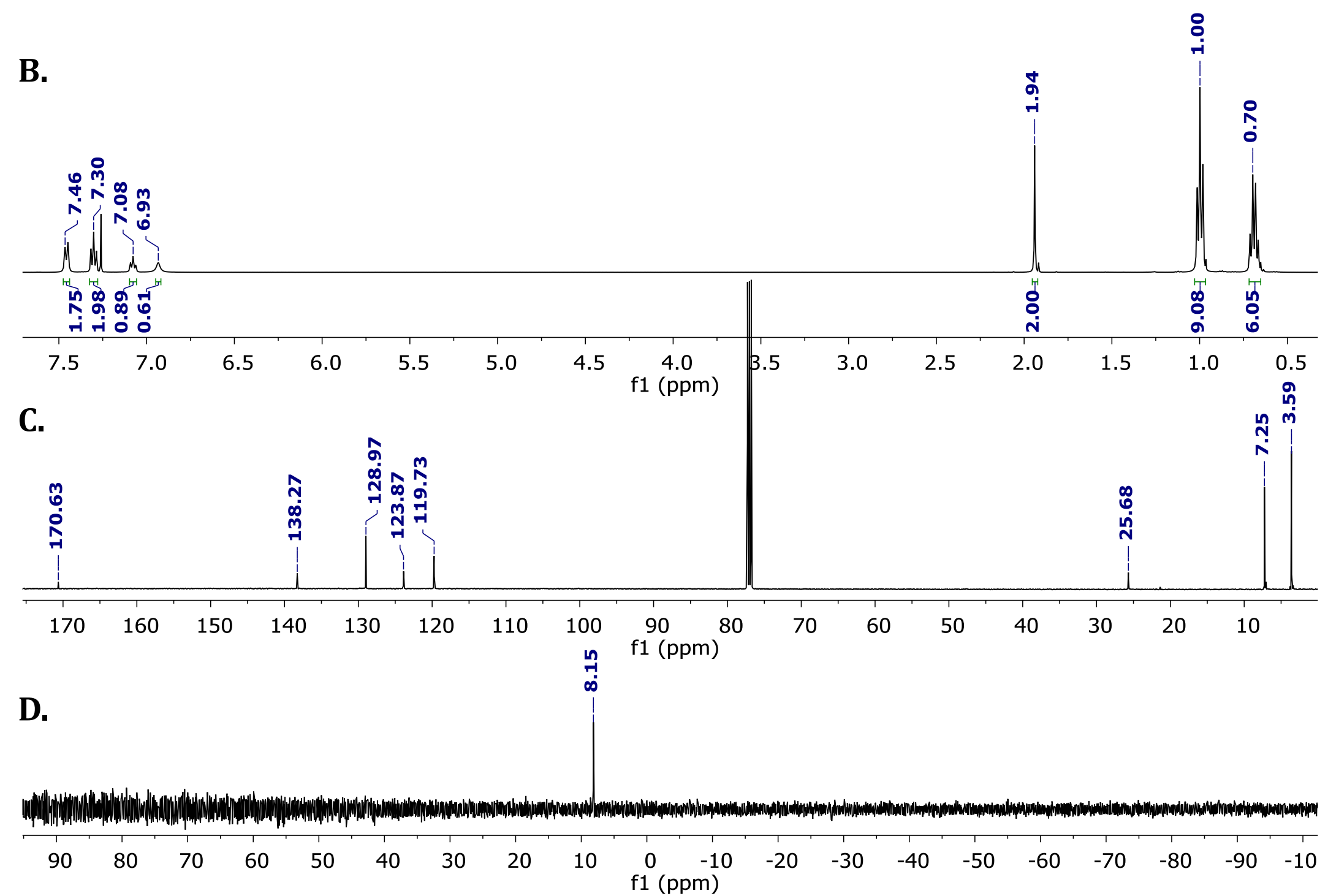


E.

09241911 \#95-103 RT: 0.42-0.46 AV: 9 SB: 13 0.19-0.24 NL: 3.86E8 T: FTMS + p ESI Full ms [50.0000-500.0000]

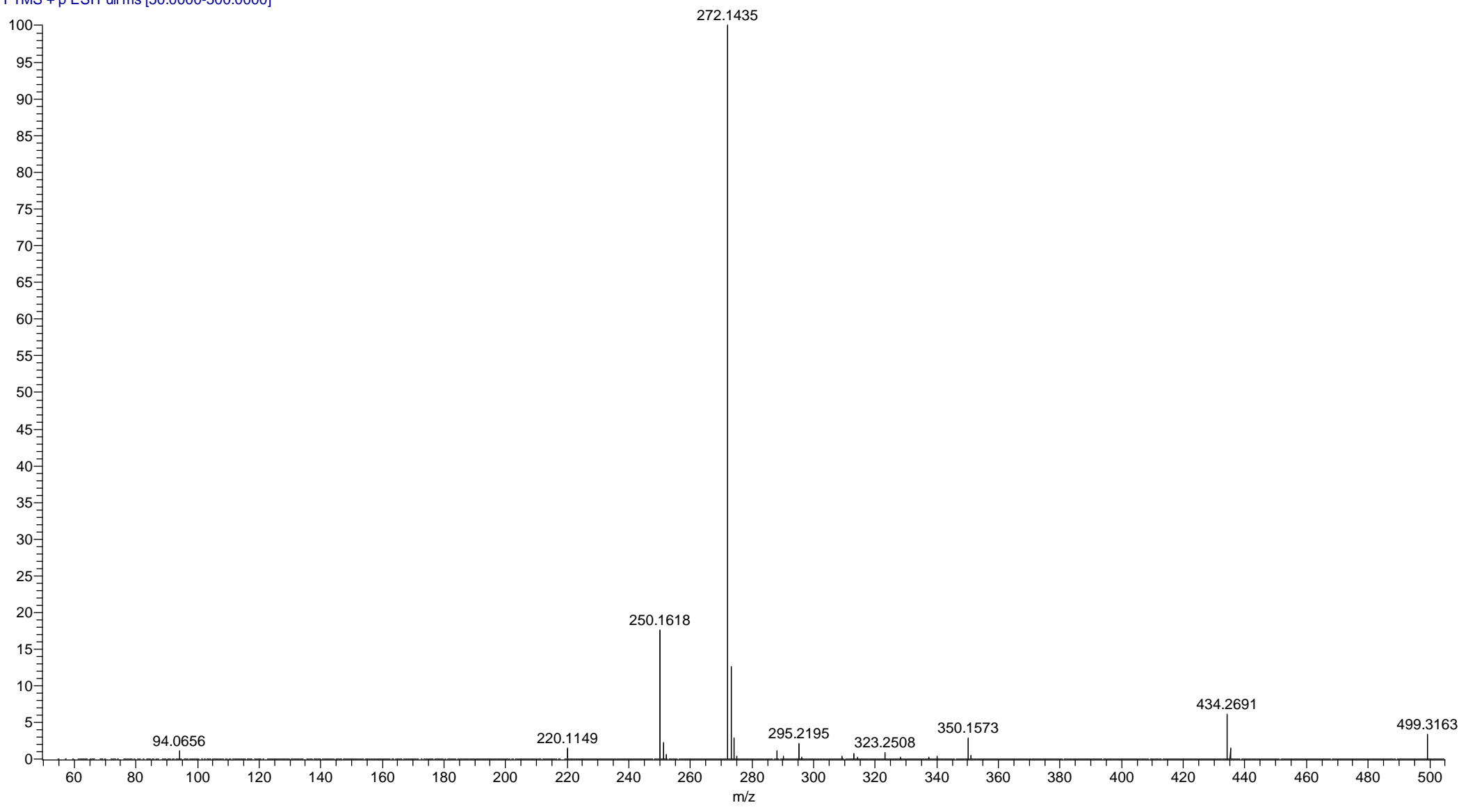


Figure S30. Compound TIPS 1 (A) FTIR (B) ${ }^{1} \mathrm{H}$ NMR (C) ${ }^{13} \mathrm{C}$ NMR (D) ${ }^{29}$ Si NMR (E) mass spectroscopy<smiles>COc1ccc(OC(=O)C[Si](C(C)C)(C(C)C)C(C)C)cc1</smiles>

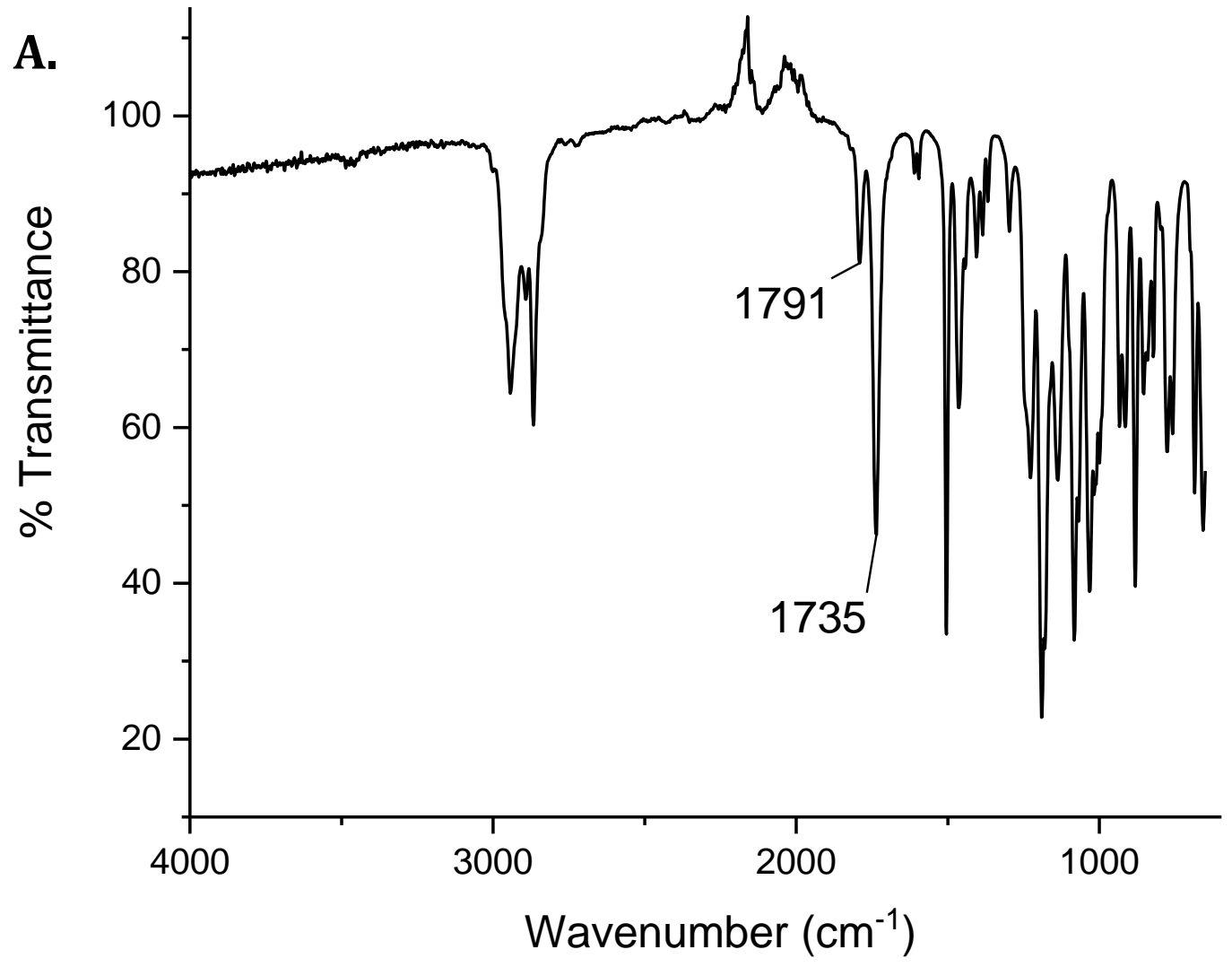




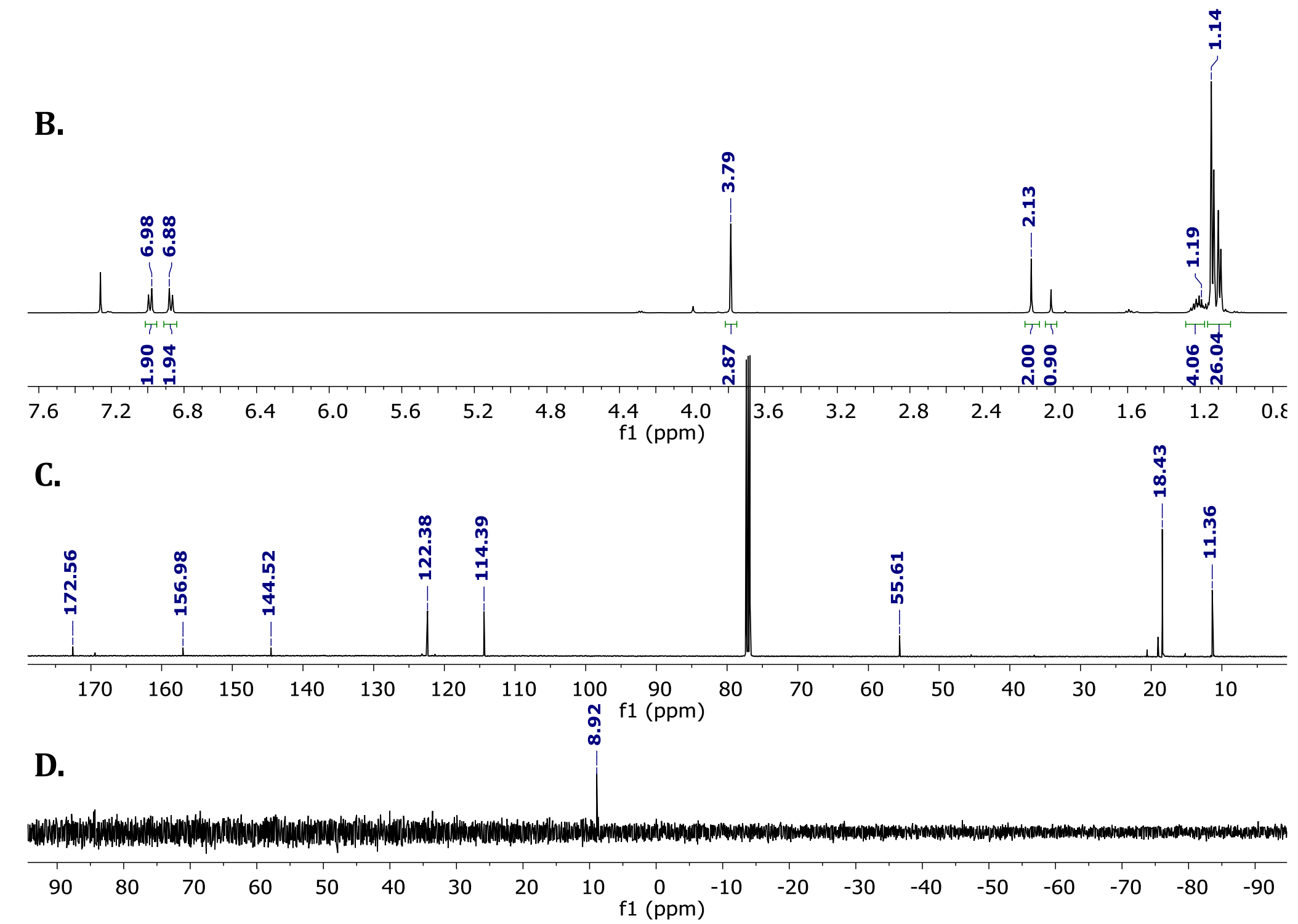


E.

09241912 \#102-120 RT: 0.45-0.53 AV: 19 SB: 17 0.23-0.30 NL: 4.44E7

T: FTMS + p ESI Full ms [50.0000-500.0000]

100 ユ

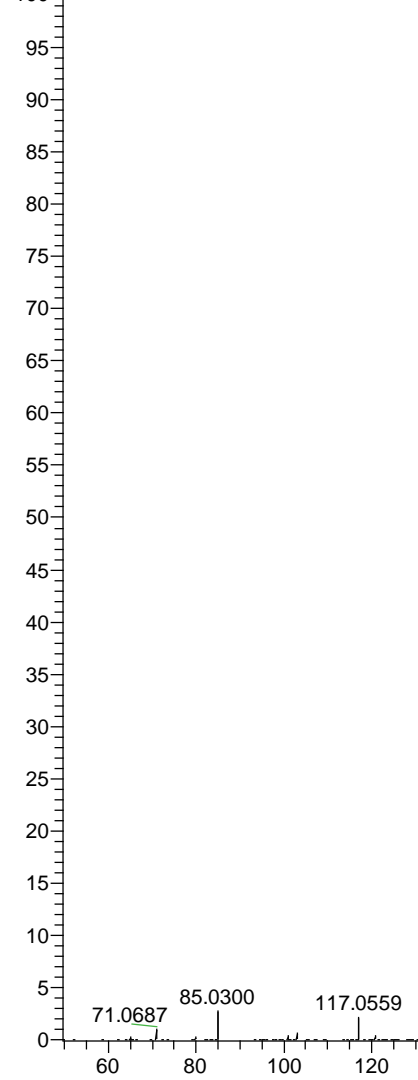

345.1852

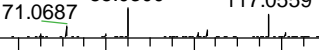

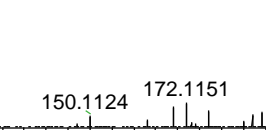

234.1882

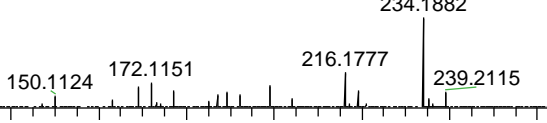


Figure S31. Compound TIPS 2 (A) FTIR (B) ${ }^{1} \mathrm{H}$ NMR (C) ${ }^{13} \mathrm{C}$ NMR (D) ${ }^{29}$ Si NMR (E) mass spectroscopy<smiles>COc1ccc(SC(=O)C[Si](C(C)C)(C(C)C)C(C)C)cc1</smiles>

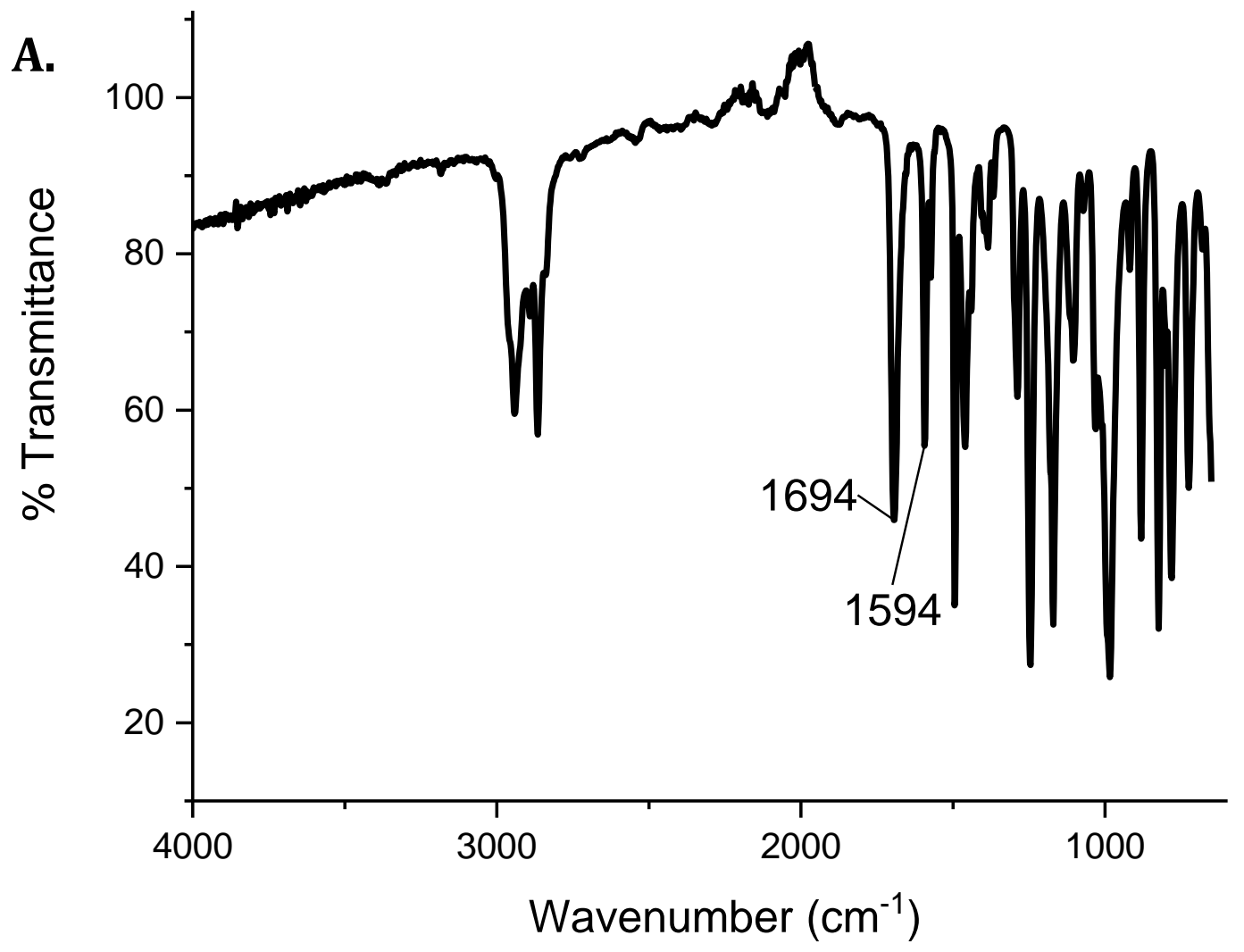




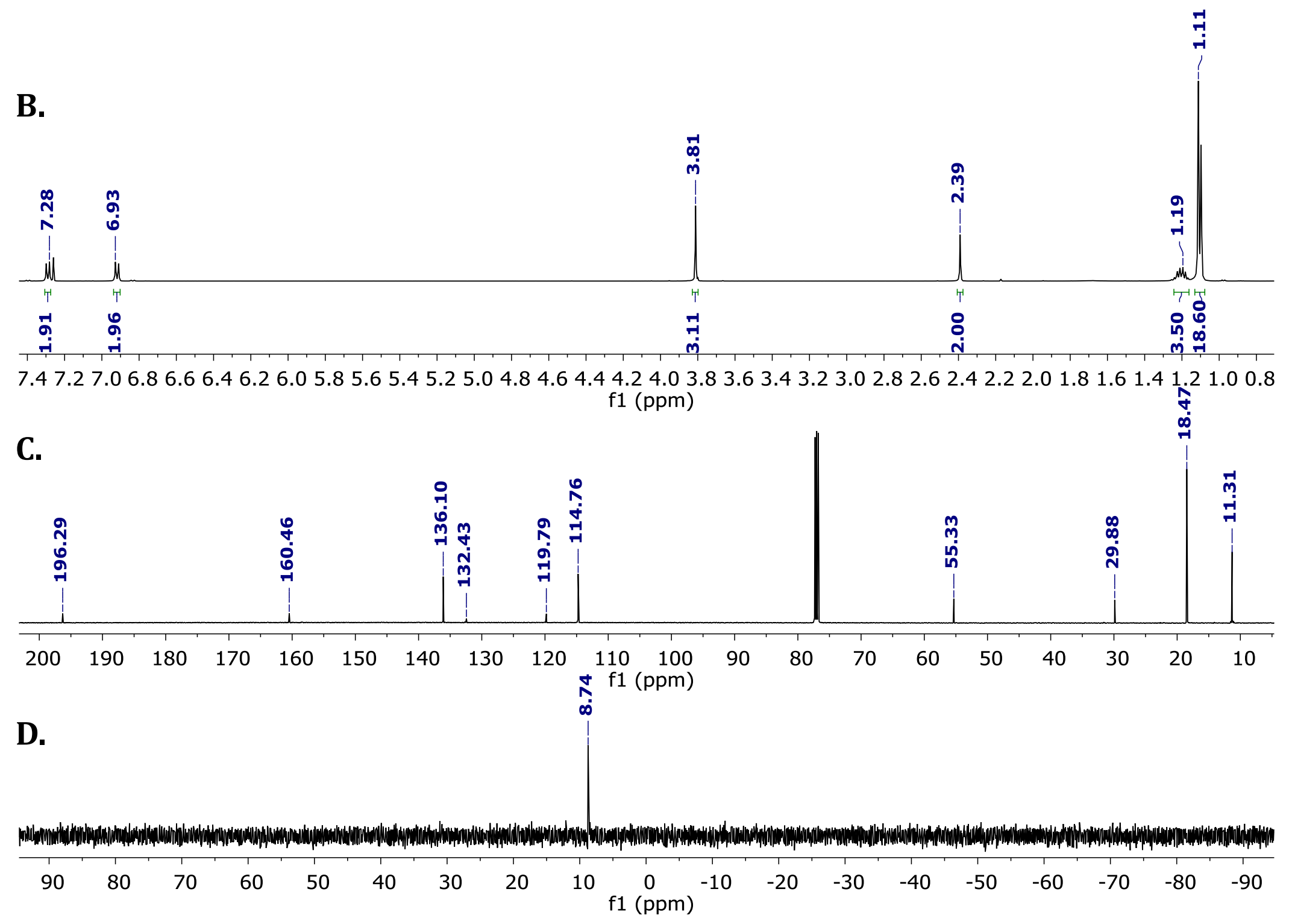


compound TIPS 2

E. 09241913\#132-139 RT: 0.59-0.62 AV: 8 SB: 22 0.05-0.14 NL: 7.39E7

${ }^{100} \mathrm{~F}$

95 年

90 年

80 年

$75^{3}$

70 寻

65

60 年

55

45격

${ }^{40} \mathrm{\exists}$

35 年

30

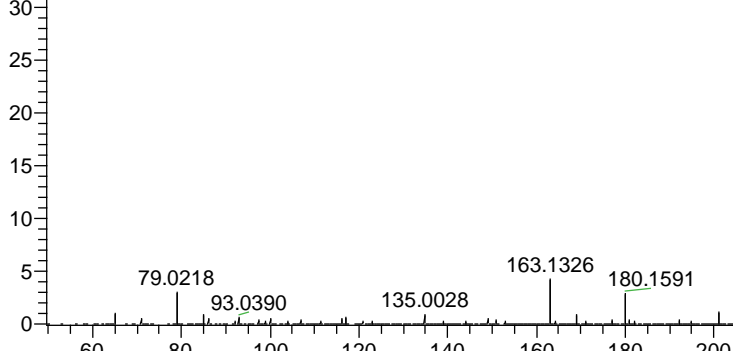

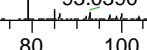
$+\frac{1}{1+1}$

180

$\frac{1}{200}$

361.1621

339.1804

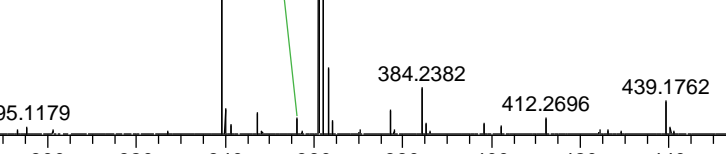


Figure S32. Compound TIPS 3 (A) FTIR (B) ${ }^{1} \mathrm{H}$ NMR (C) ${ }^{13} \mathrm{C}$ NMR (D) ${ }^{29}$ Si NMR (E) mass spectroscopy<smiles>CC(C)[Si](CC(=O)Nc1ccccc1)(C(C)C)C(C)C</smiles>

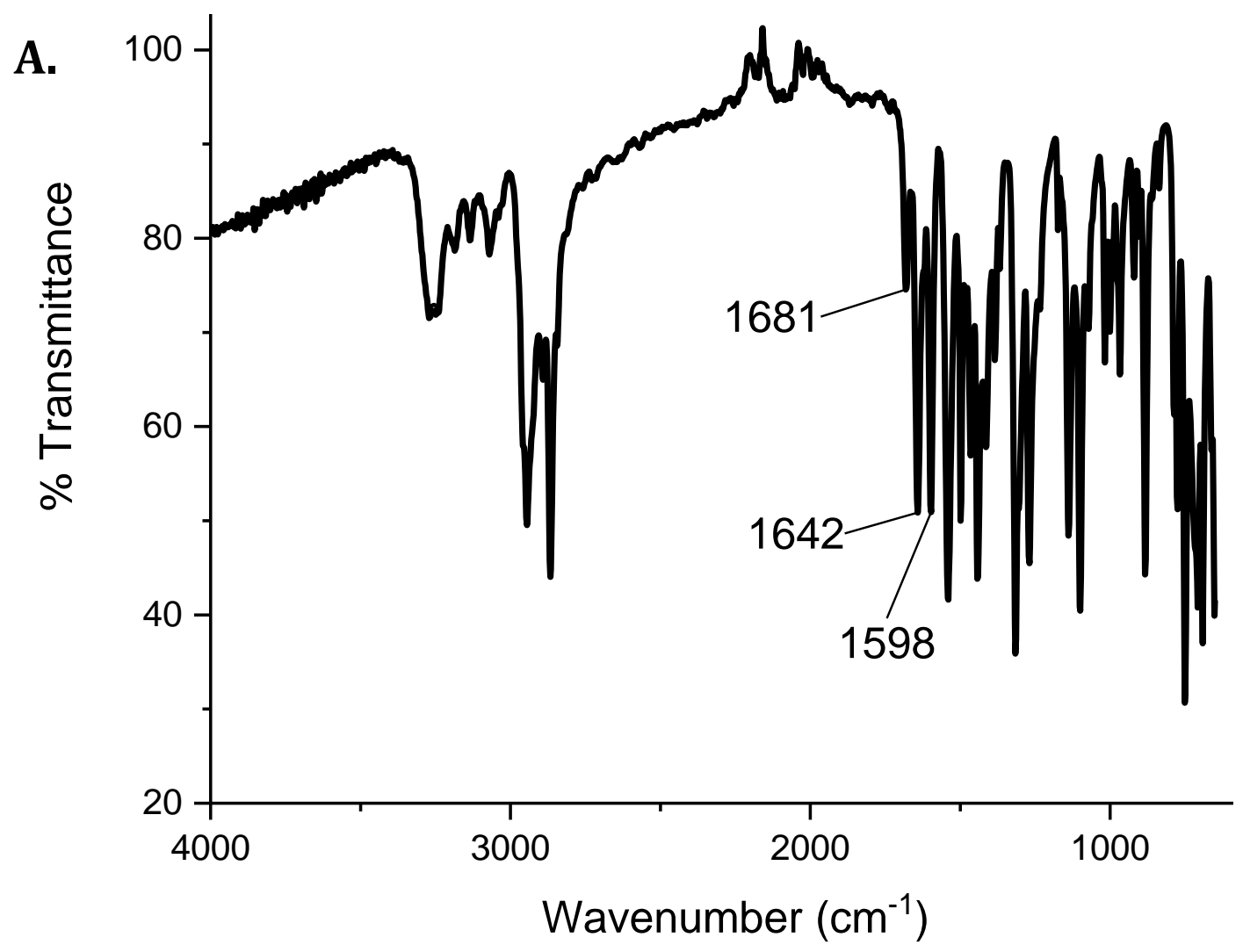




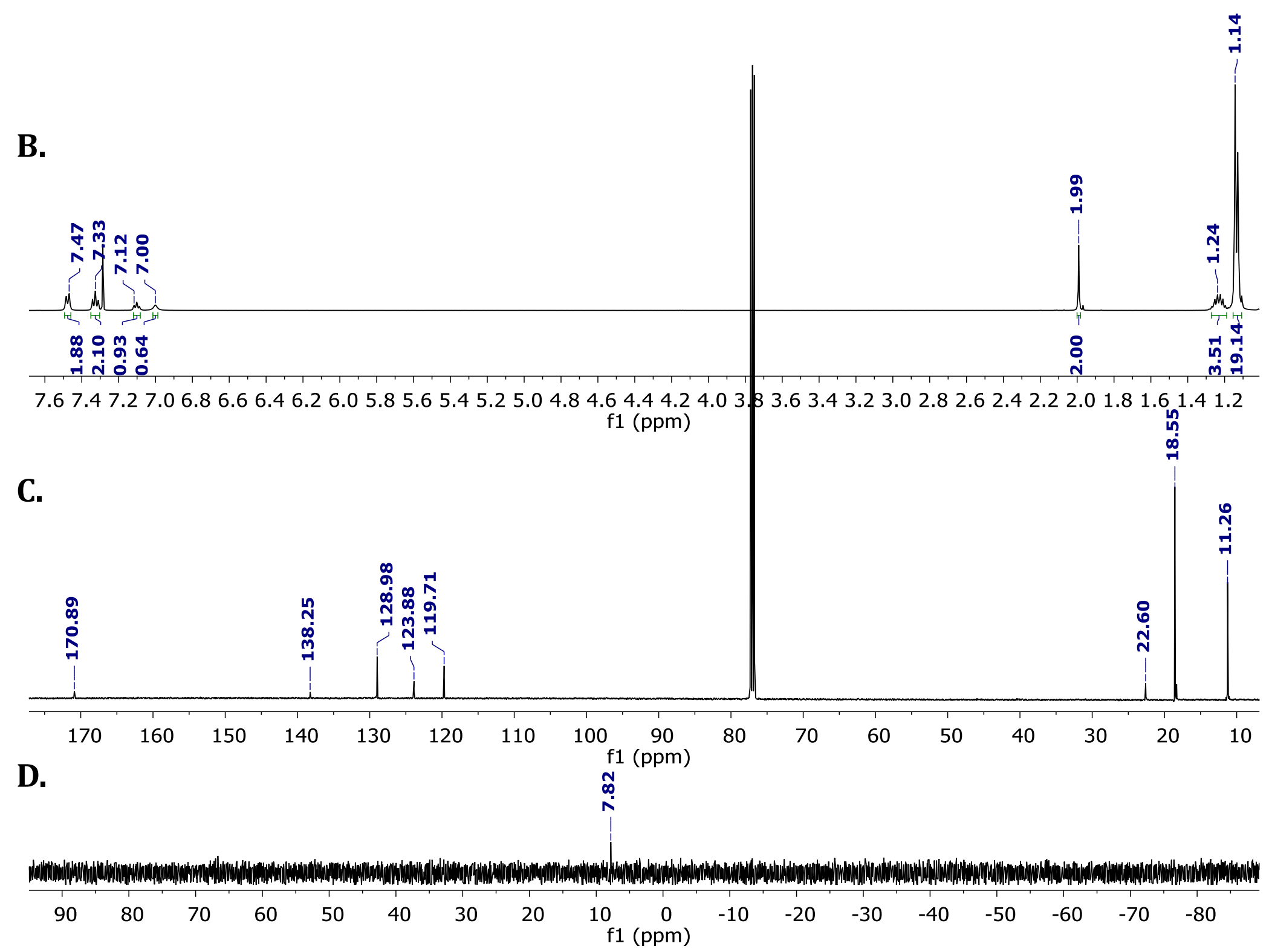


E.

09241914 \#101-116 RT: $0.45-0.52 \quad$ AV: 16 SB: 24 0.31-0.41 NL: 1.10E8
T: FTMS + p ESI Full ms [50.0000-500.0000]

95

$8{ }^{9} \mathrm{~A}$

$85 \exists$

80

70

65

60

55

50

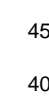

30

30

25

$20=$

15

10

10
5
0

85.0300

$\begin{array}{lrr}117.0558 & 169.1409 \quad 201.0886\end{array}$

314.1904

${ }_{60}{ }_{80} 100 \quad 120$

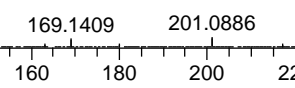

292.2086

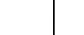

337.2664

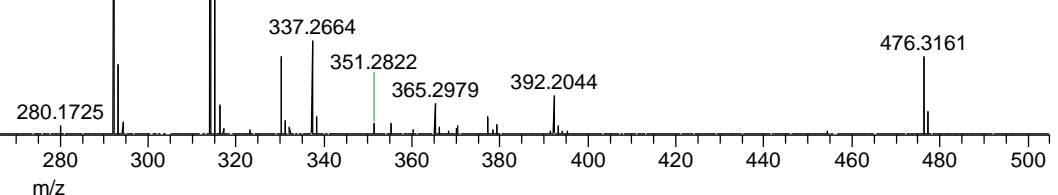


Figure S33. Compound DMPhS 1 (A) FTIR (B) ${ }^{1} \mathrm{H}$ NMR (C) ${ }^{13} \mathrm{C}$ NMR (D) ${ }^{29}$ Si NMR (E) mass spectroscopy<smiles>COc1ccc(OC(=O)C[Si](C)(C)c2ccccc2)cc1</smiles>

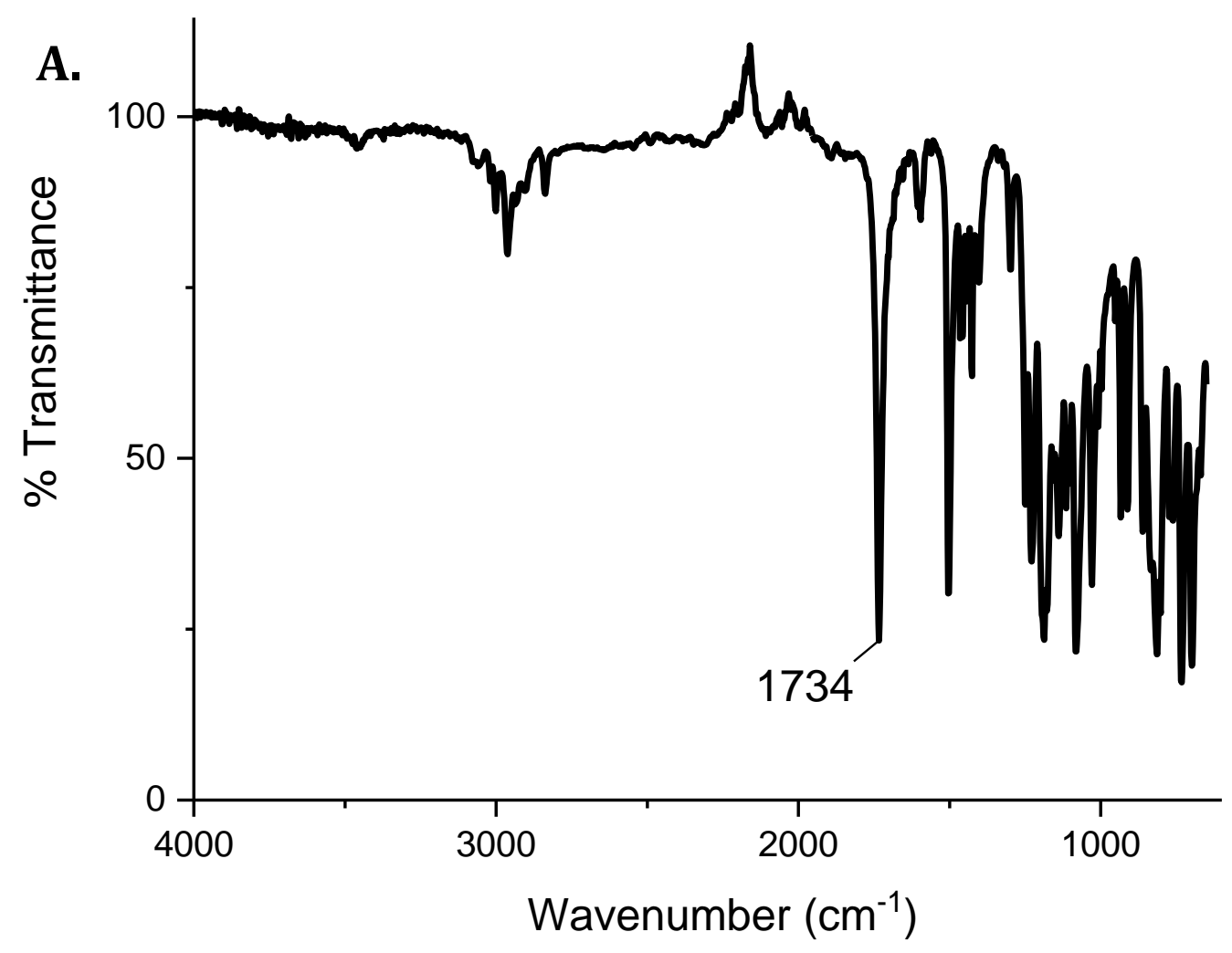


compound DMPhS 1

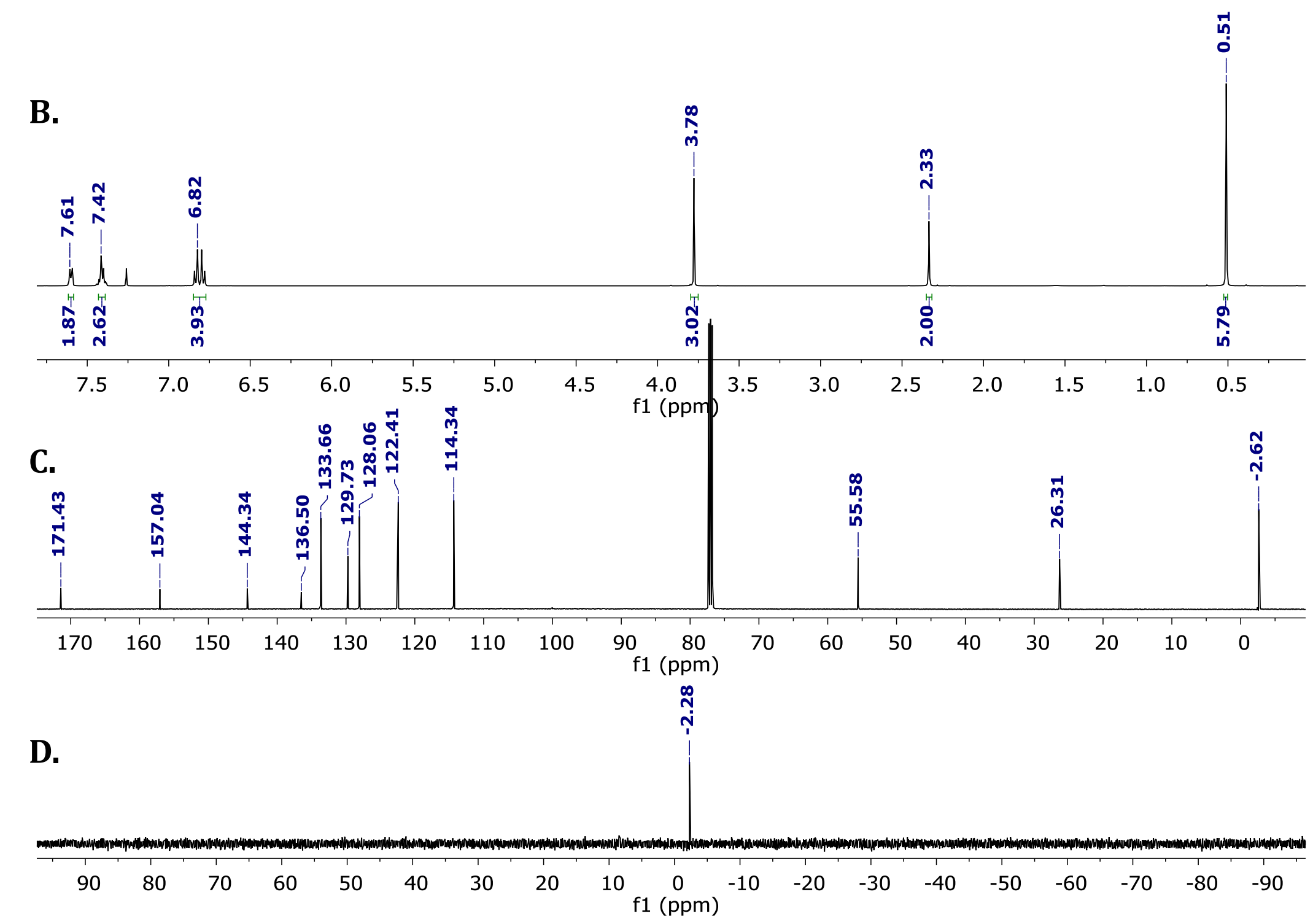


E.

09241915 \#93-104 RT: 0.41-0.46 AV: 12 SB: 27 0.28-0.40 NL: 2.13E8

9241915 \#93-104 RT: $0.41-0.46$ AV: 12
: FTMS + p ESI Full ms [50.0000-500.0000]

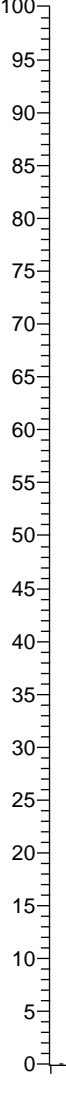

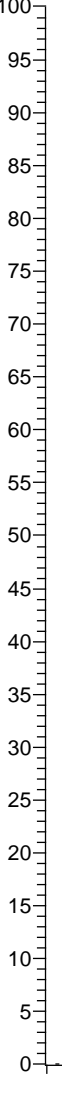

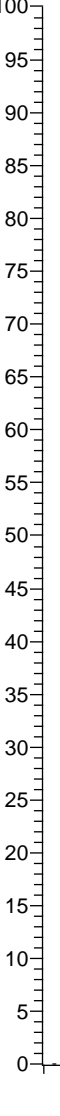

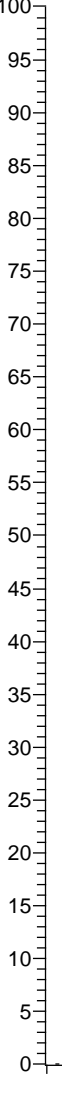

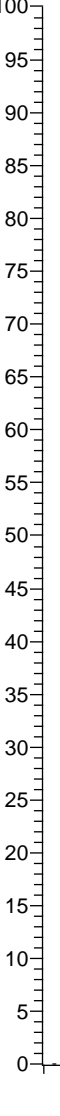

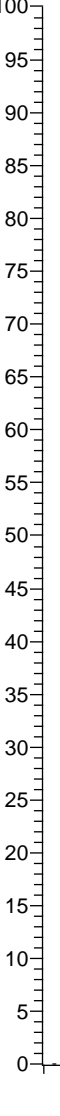

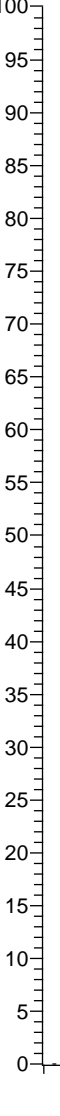

20

15

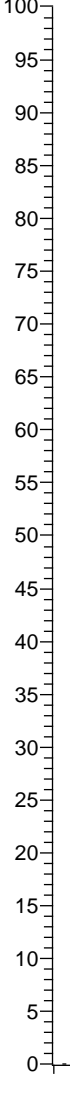

$$
\text { 10 }
$$

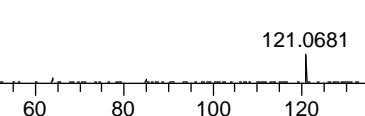

1
60
80
323.1069

18.1516

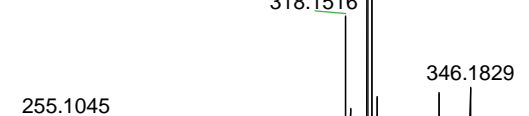

$\begin{array}{lll}6.1829 & 374.2142 \quad 401.1209\end{array}$

T) 220

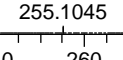

280

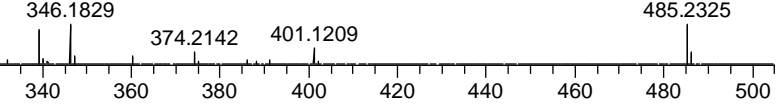


Figure S34. Compound DMPhS 2 (A) FTIR (B) ${ }^{1} \mathrm{H}$ NMR (C) ${ }^{13} \mathrm{C}$ NMR (D) ${ }^{29}$ Si NMR (E) mass spectroscopy<smiles>COc1ccc(SC(=O)C[Si](C)(C)c2ccccc2)cc1</smiles>

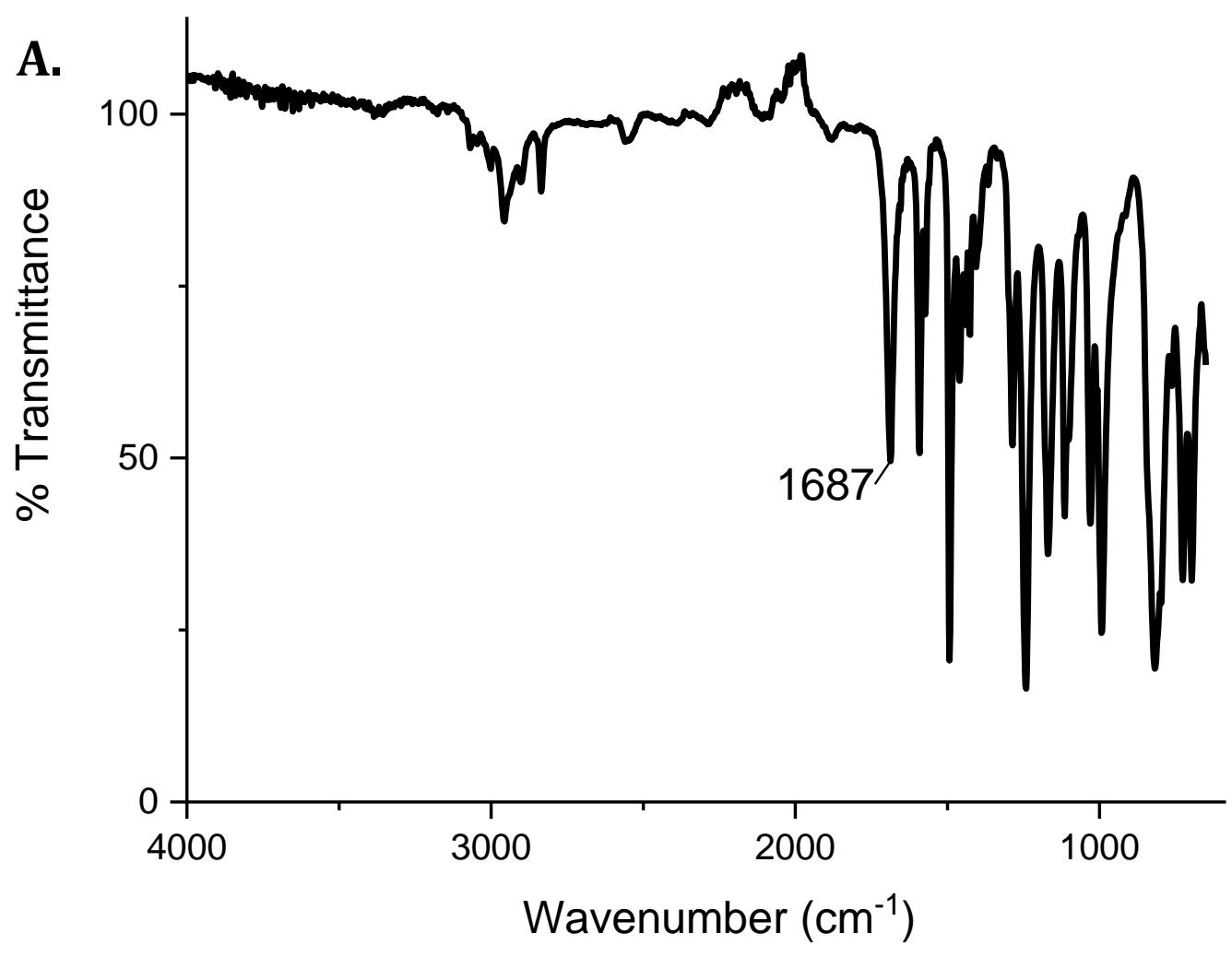


compound DMPhS 2

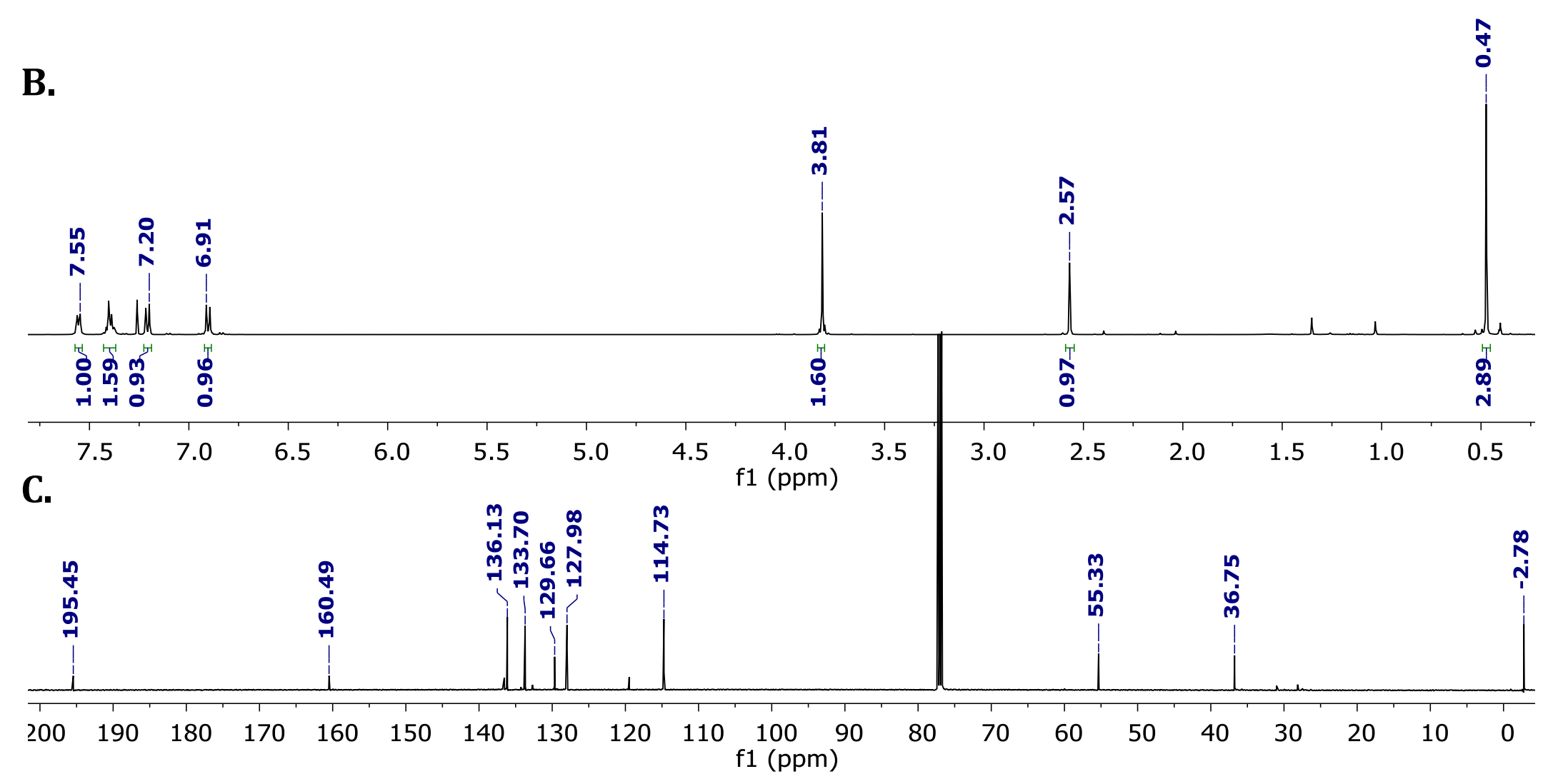

D.

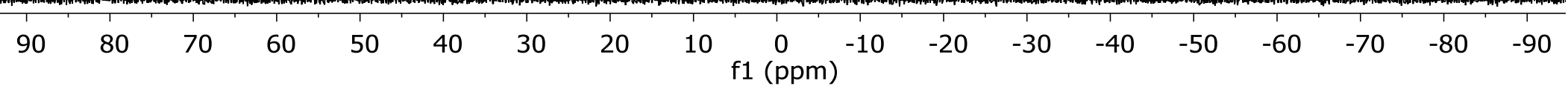


E.

09241916 \#96-108 RT: $0.43-0.48$ AV: 13 SB: 19 0.34-0.42 NL: 1.08E8
T: FTMS + p ESI Full ms [50.0000-500.0000]

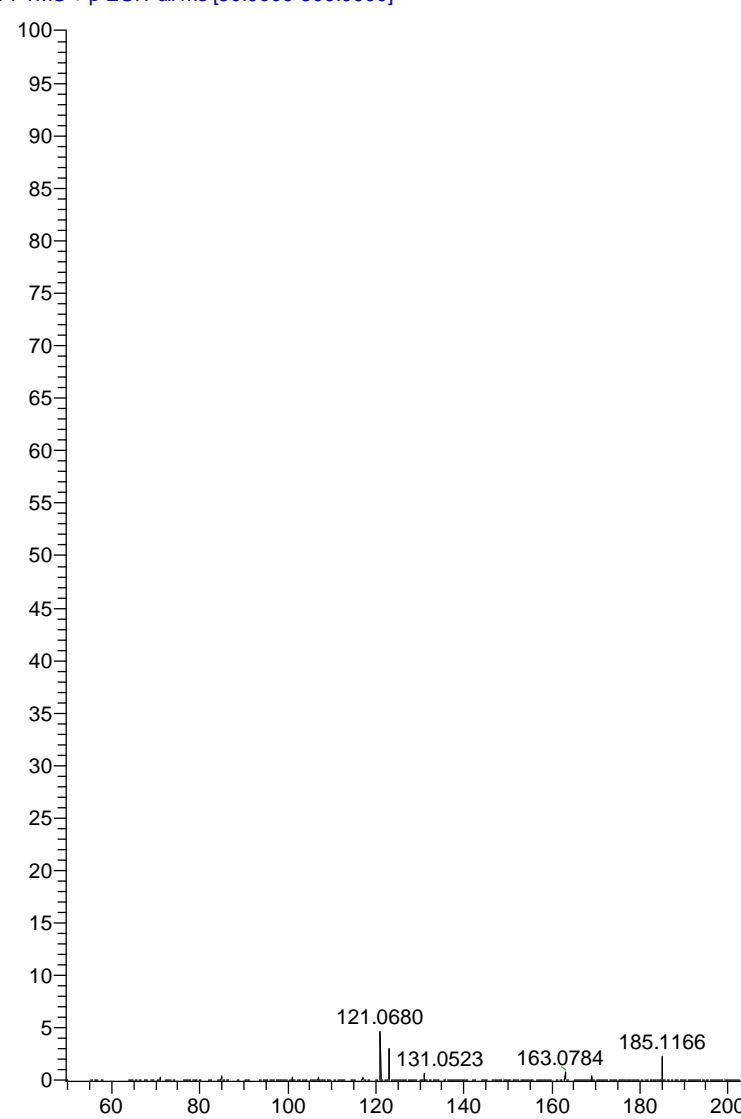

339.0840

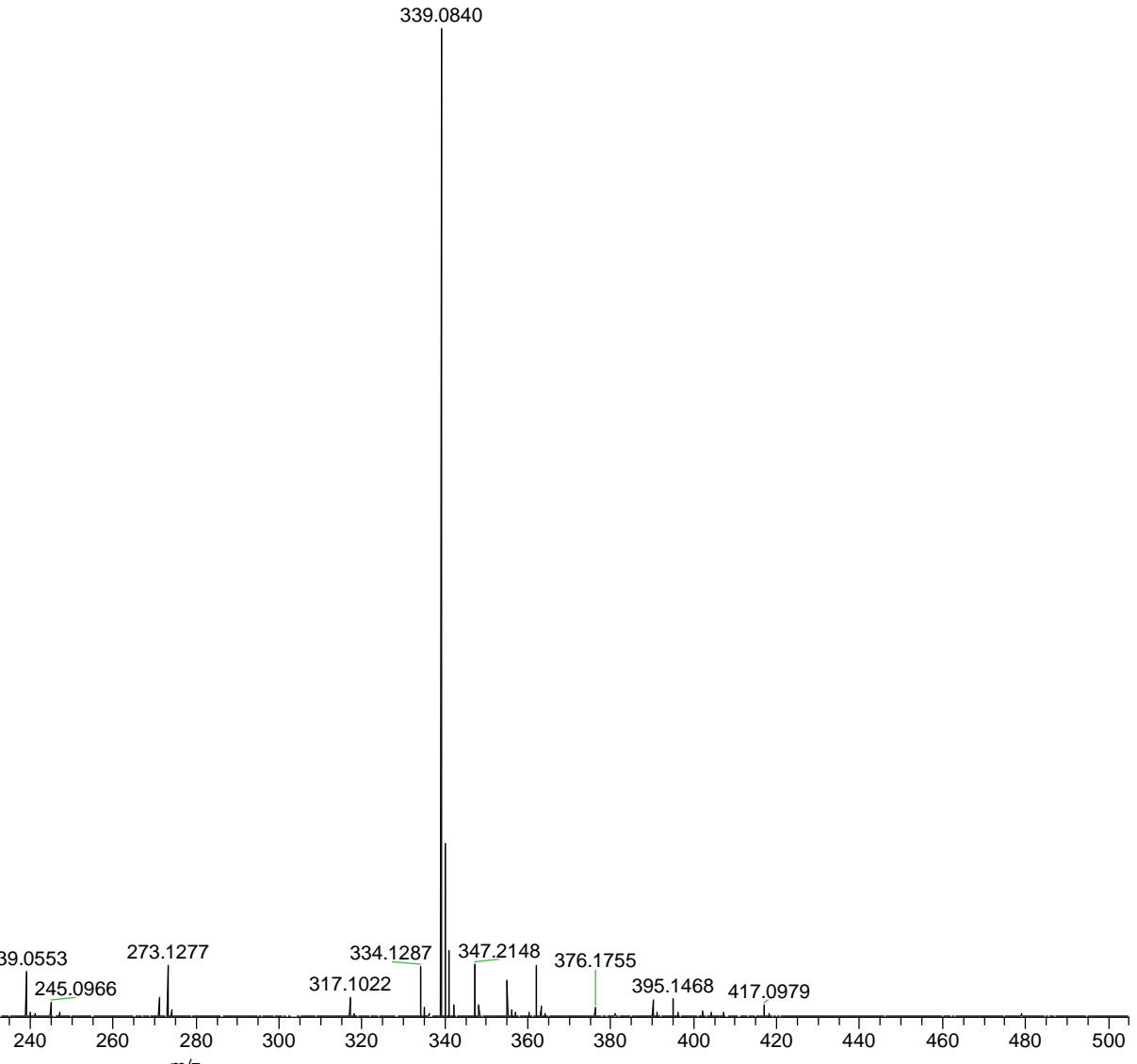


Figure S35. Compound DMPhS 3 (A) FTIR (B) ${ }^{1} \mathrm{H}$ NMR (C) ${ }^{13} \mathrm{C}$ NMR (D) ${ }^{29}$ Si NMR (E) mass spectroscopy<smiles>C[Si](C)(CC(=O)Nc1ccccc1)c1ccccc1</smiles>

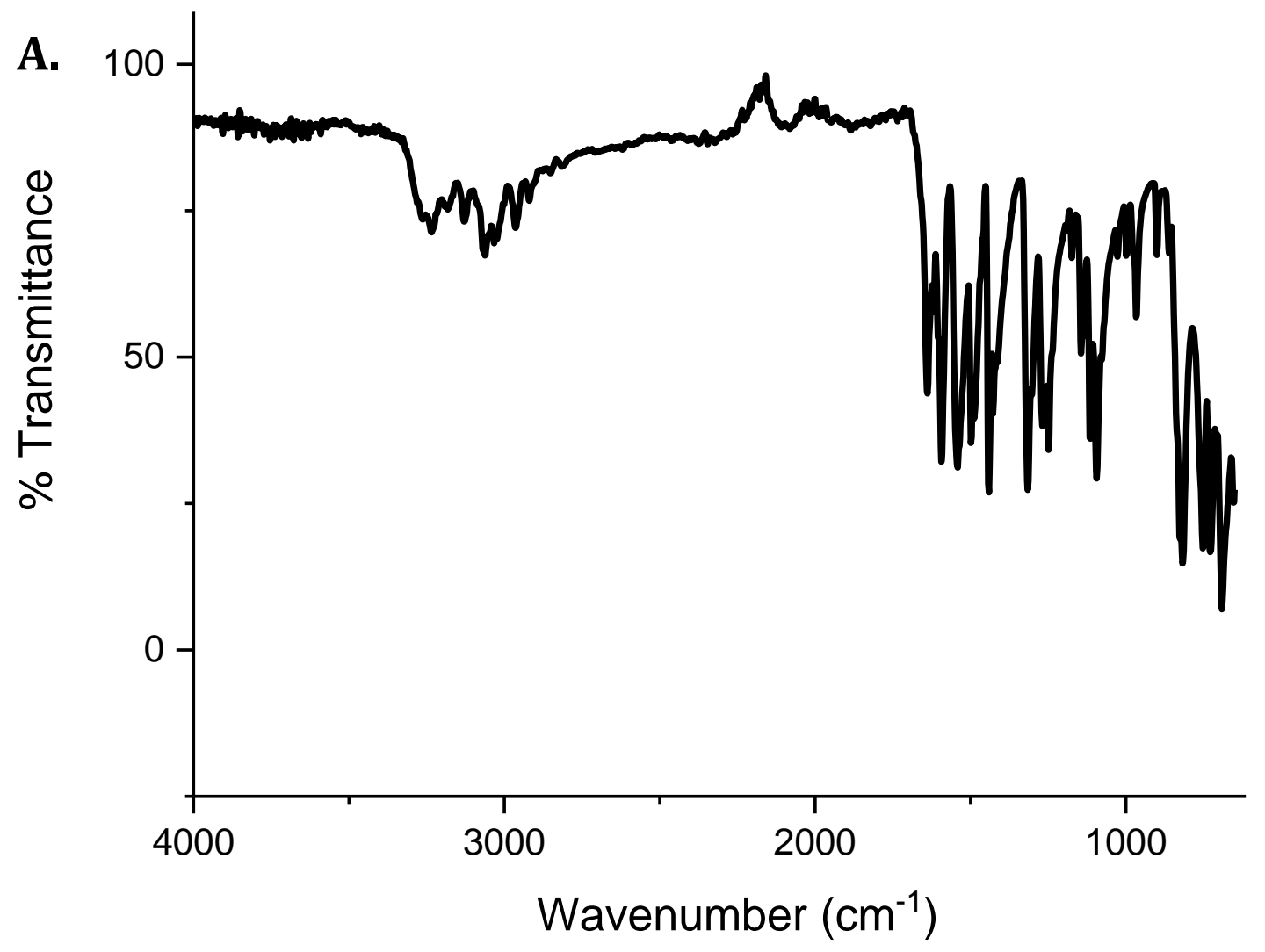


B.

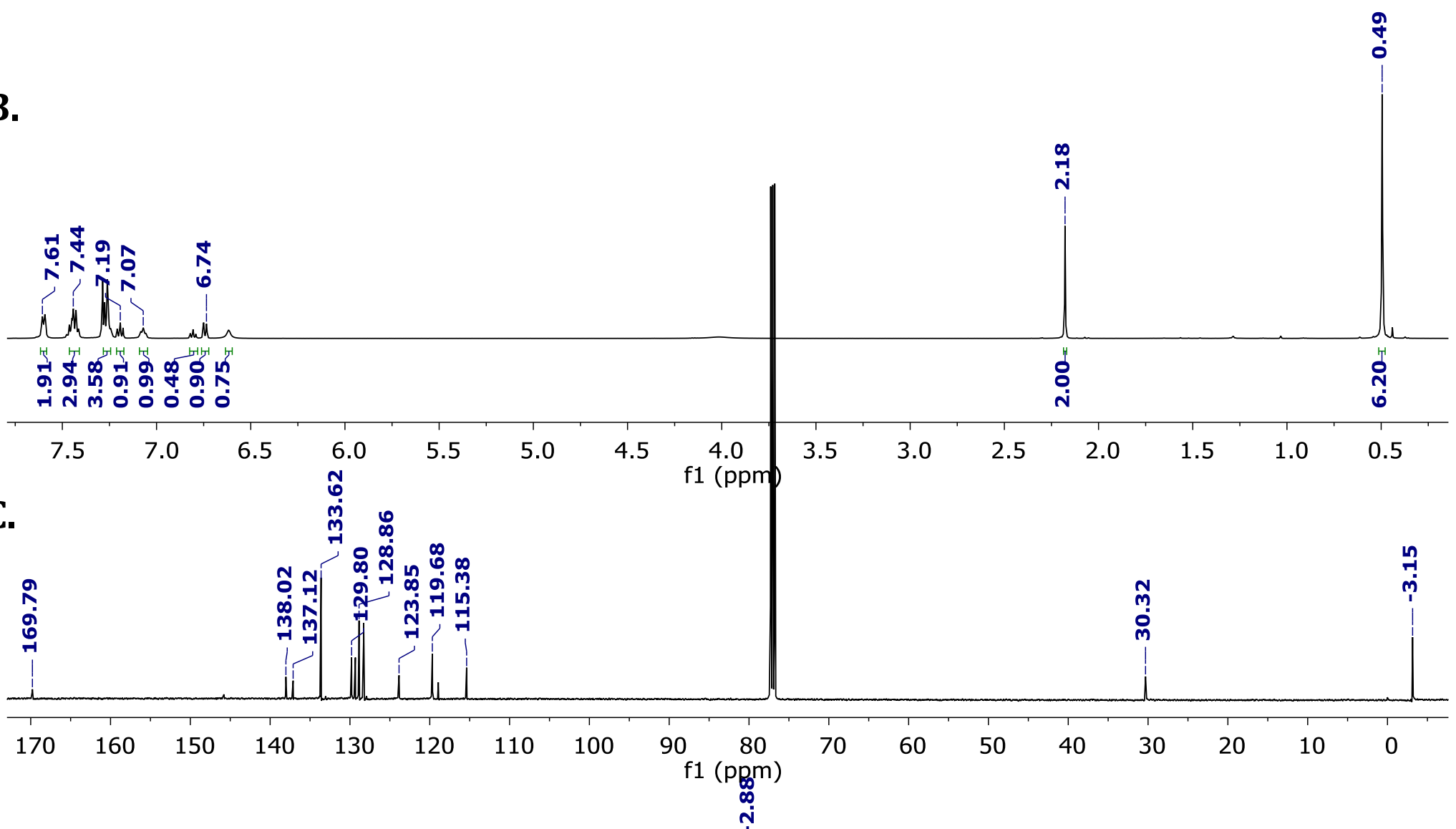

D.

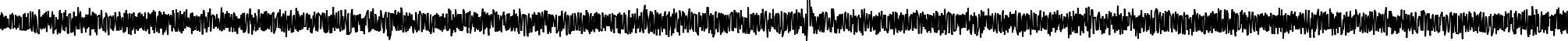

60

$\begin{array}{lll}50 & 40 \quad 30\end{array}$

20

$\mathrm{f} 1(\mathrm{ppm})^{-10}$

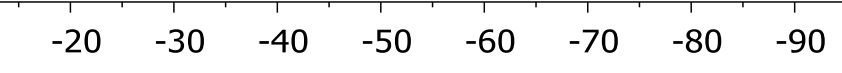


compound DMPhS 3

E. 09241917 \#98-105 RT: 0.44-0.47 AV: 8 SB: 31 0.29-0.43 NL: 1.90E8

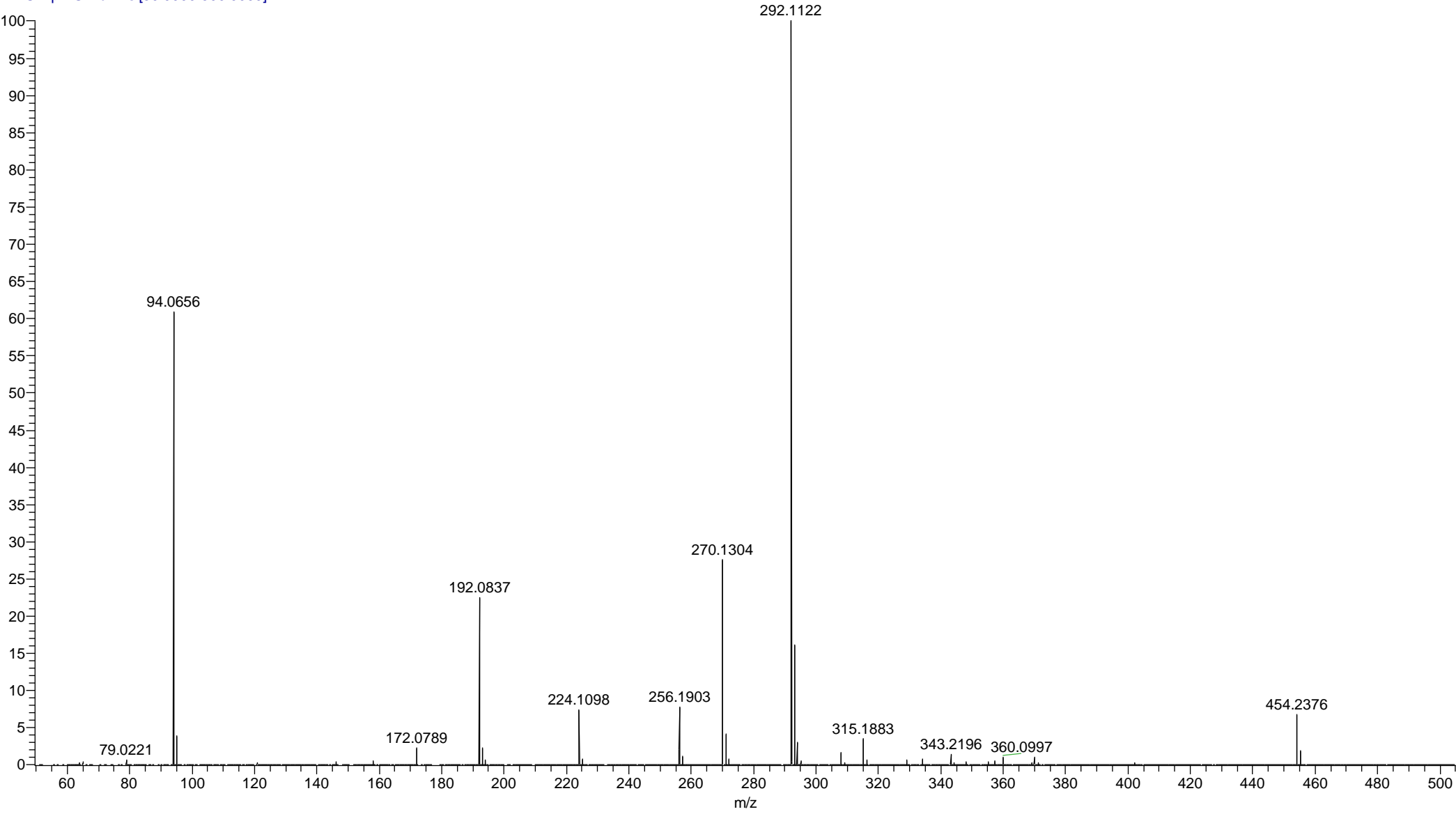


Figure S36. Compound TPhS 1 (A) FTIR (B) ${ }^{1} \mathrm{H}$ NMR (C) ${ }^{13} \mathrm{C}$ NMR (D) ${ }^{29} \mathrm{Si}$ NMR (E) mass spectroscopy<smiles>[R4]C(C)=[W]N(C)C(=O)C[Si](c1ccccc1)(c1ccccc1)c1ccccc1</smiles>

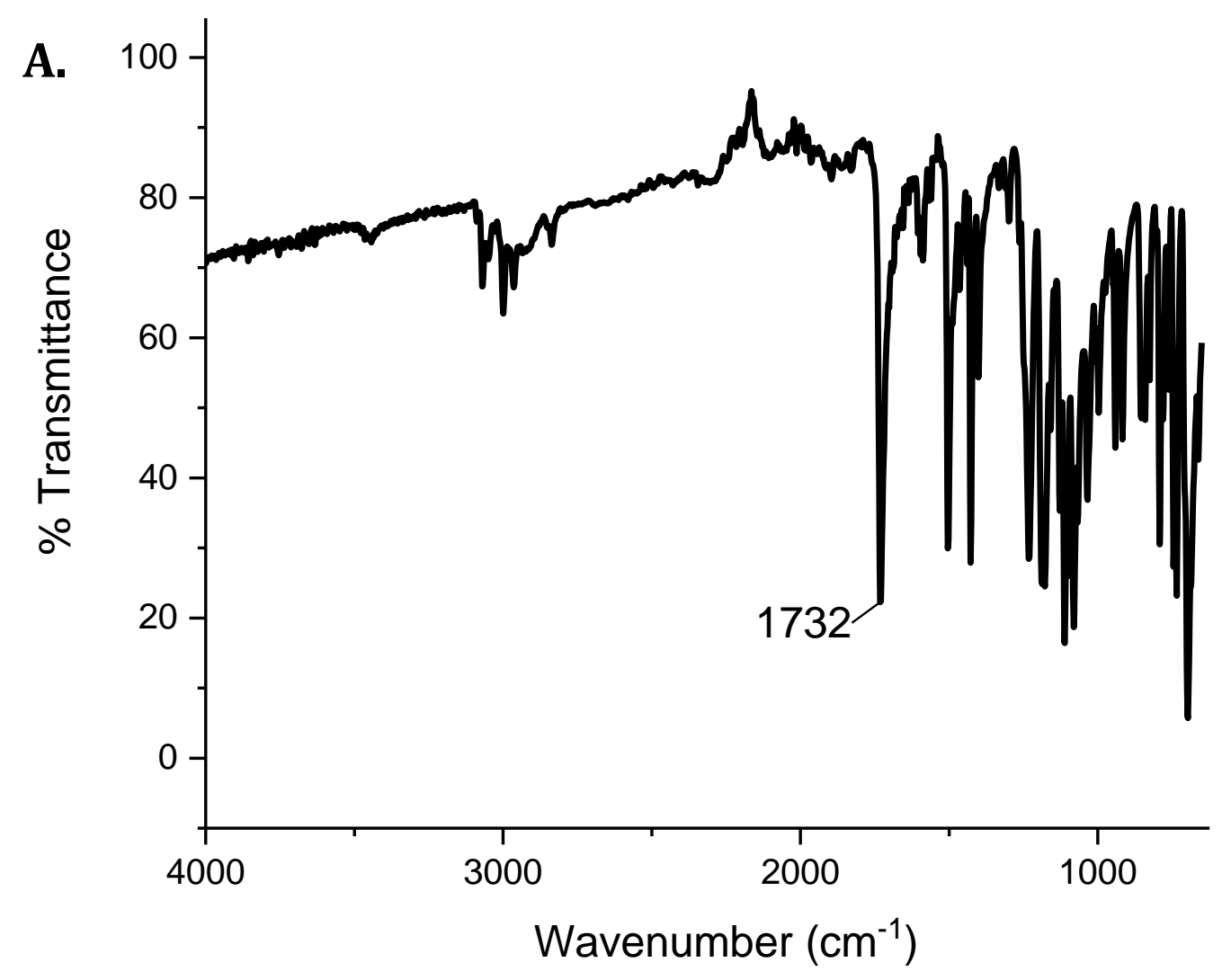




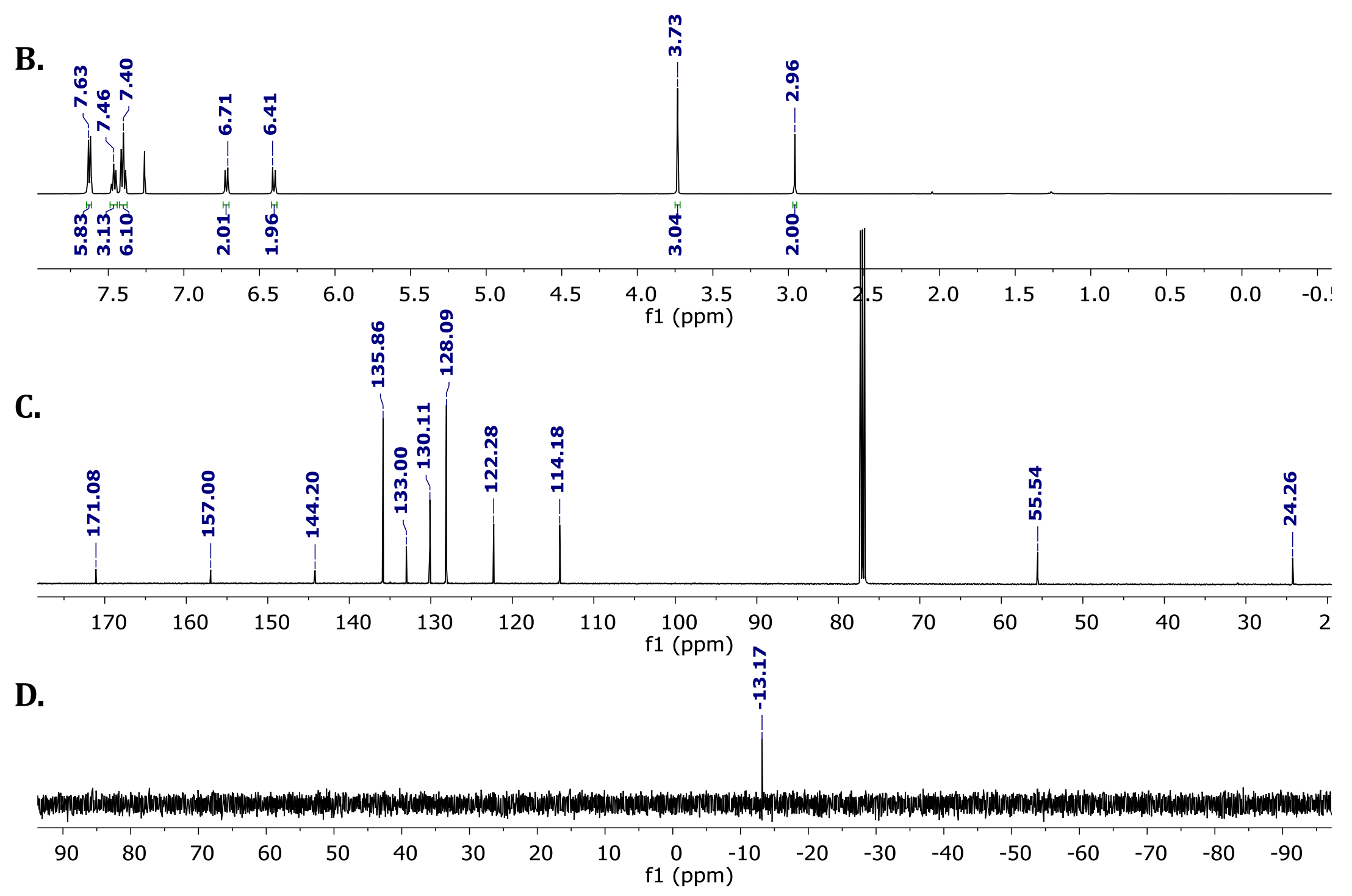


E.

09241918 \#95-102 RT: 0.42-0.45 AV: 8 SB: 6 0.20-0.22 NL: 1.96E7 T: FTMS + p ESI Full ms [50.0000-600.0000]

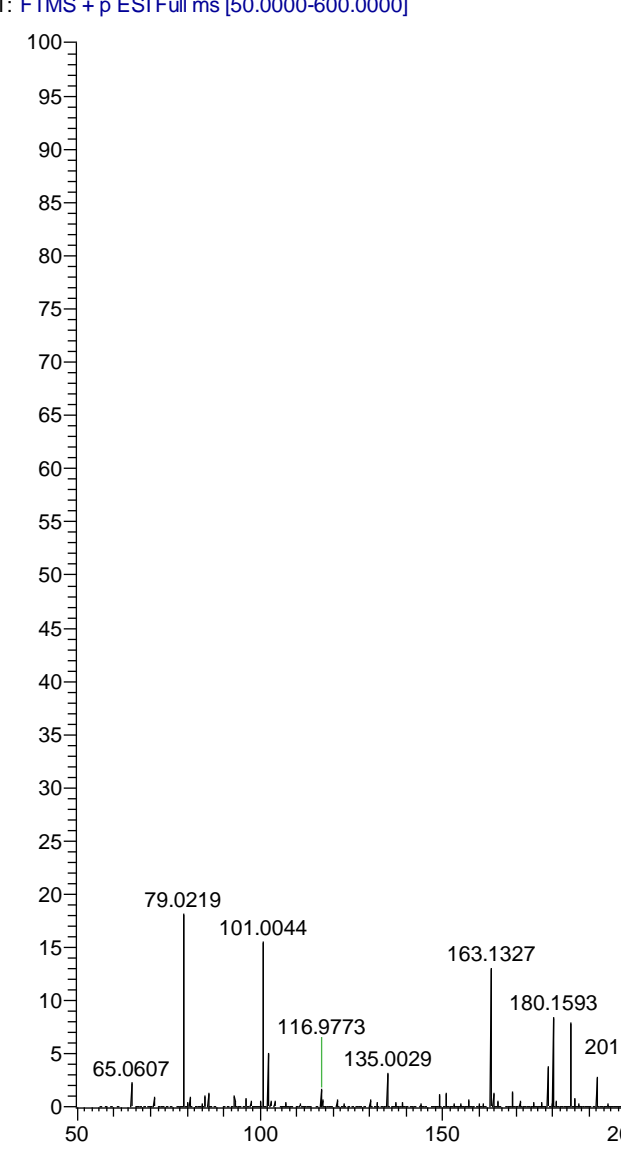

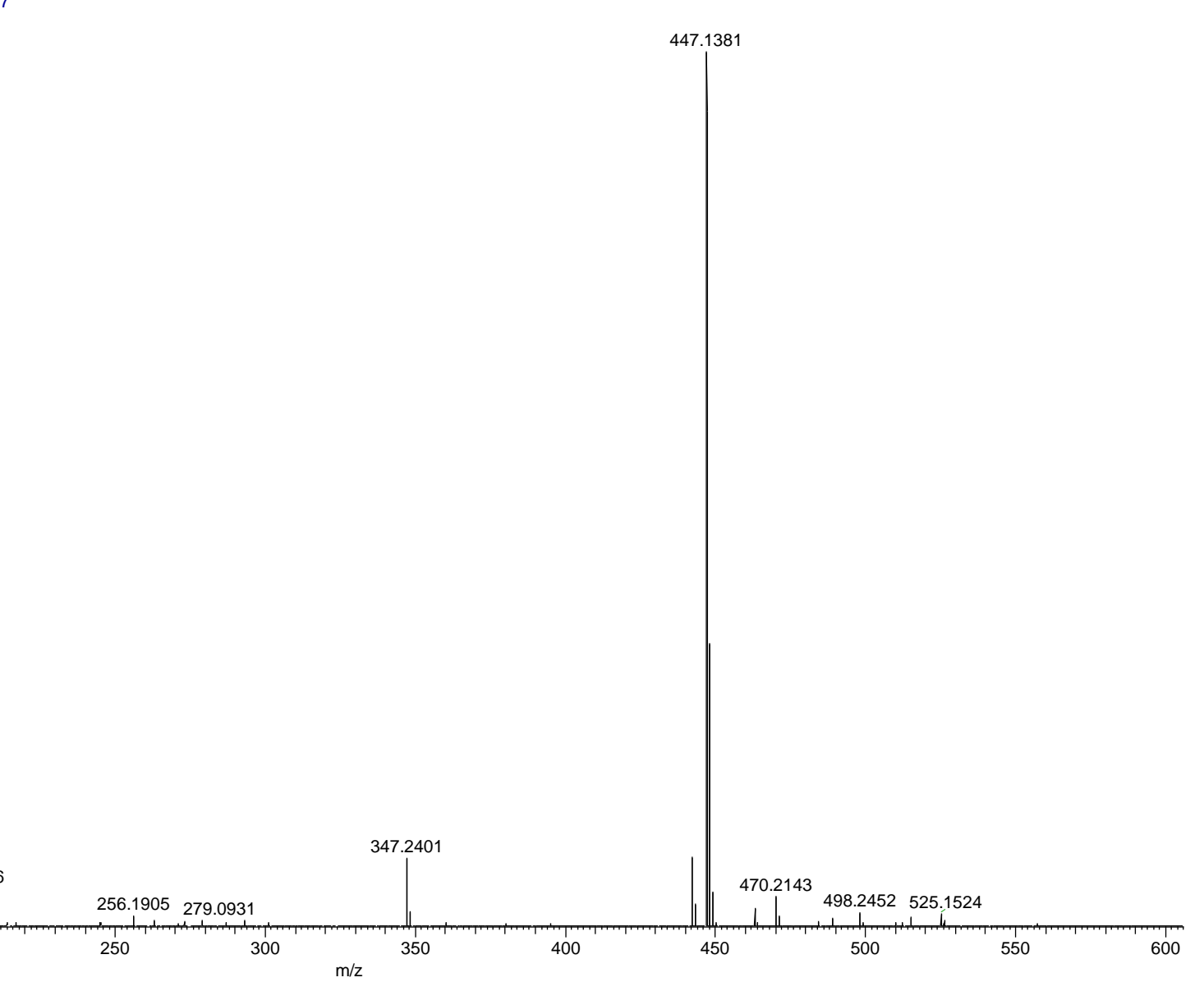


Figure S37. Compound TPhS 2 (A) FTIR (B) ${ }^{1} \mathrm{H}$ NMR (C) ${ }^{13} \mathrm{C}$ NMR (D) ${ }^{29}$ Si NMR (E) mass spectroscopy<smiles>COc1ccc(SC(=O)C[Si](c2ccccc2)(c2ccccc2)c2ccccc2)cc1</smiles>

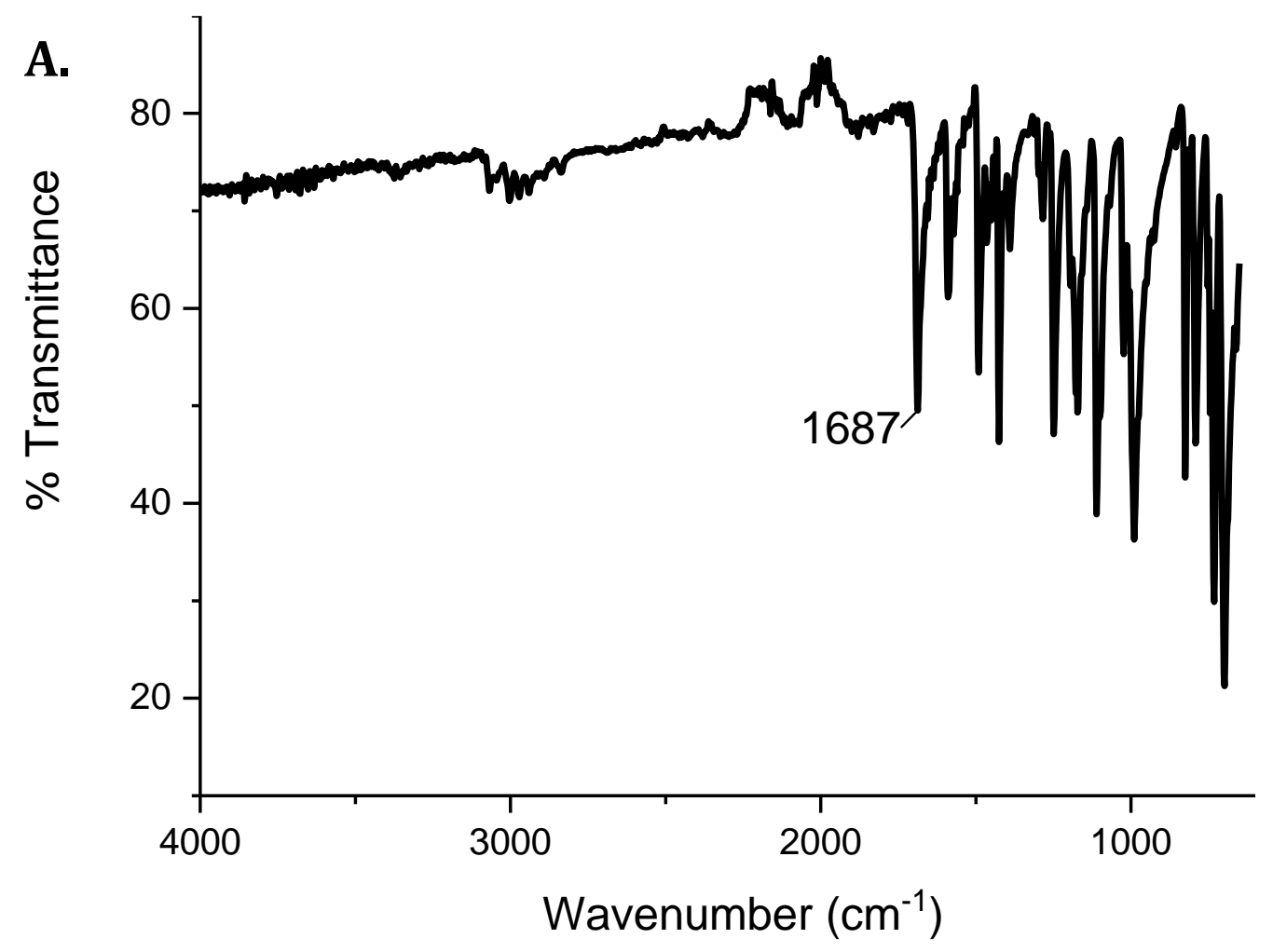




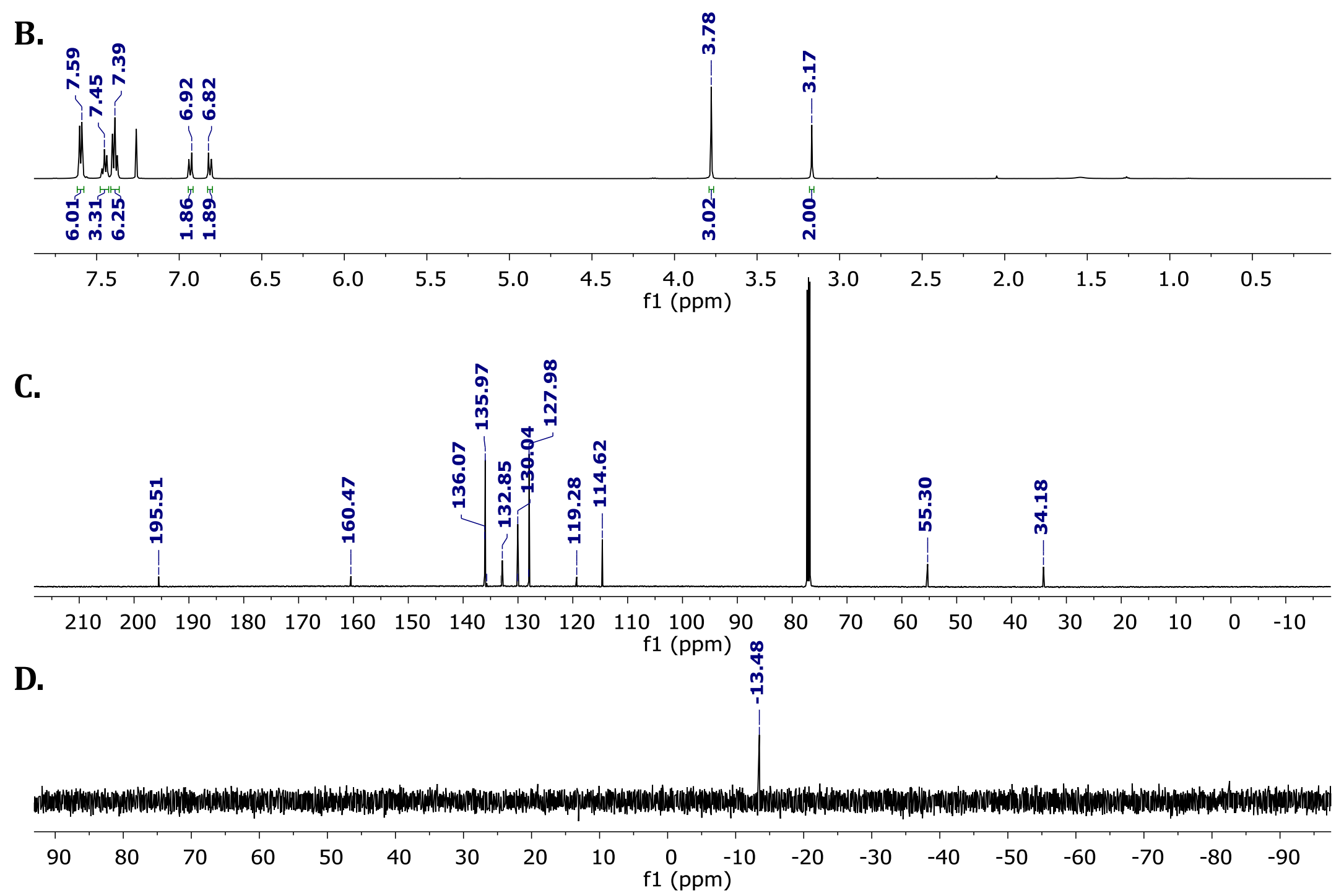


E.

09241919a \#88-93 RT: 0.39-0.41 AV: 6 SB: 18 0.00-0.04, 0.20-0.23 NL: $1.21 E 7$ T: FTMS + p ESI Full ms [50.0000-600.0000]

100 F

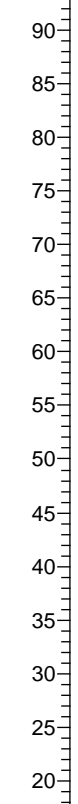

95 年

75 壮

50 年

45 当

40 年

35 年

30 年

20每

.

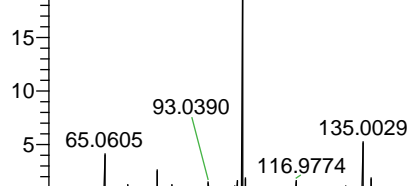

100

102.1282

$135.0029 \quad 185.1137$ 217.1046244 .9782273 .1799 200
463.1152

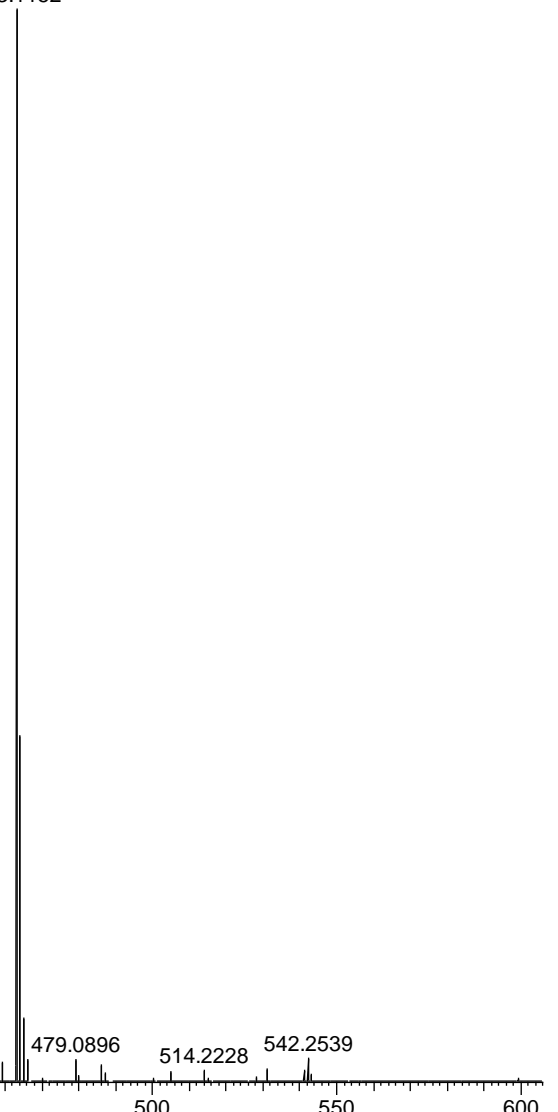


Figure S38. Compound TPhS 3 (A) FTIR (B) ${ }^{1} \mathrm{H}$ NMR (C) ${ }^{13} \mathrm{C}$ NMR (D) ${ }^{29} \mathrm{Si}$ NMR (E) mass spectroscopy<smiles>CS(=O)(=O)NCCCC(=O)Nc1ccccc1</smiles>

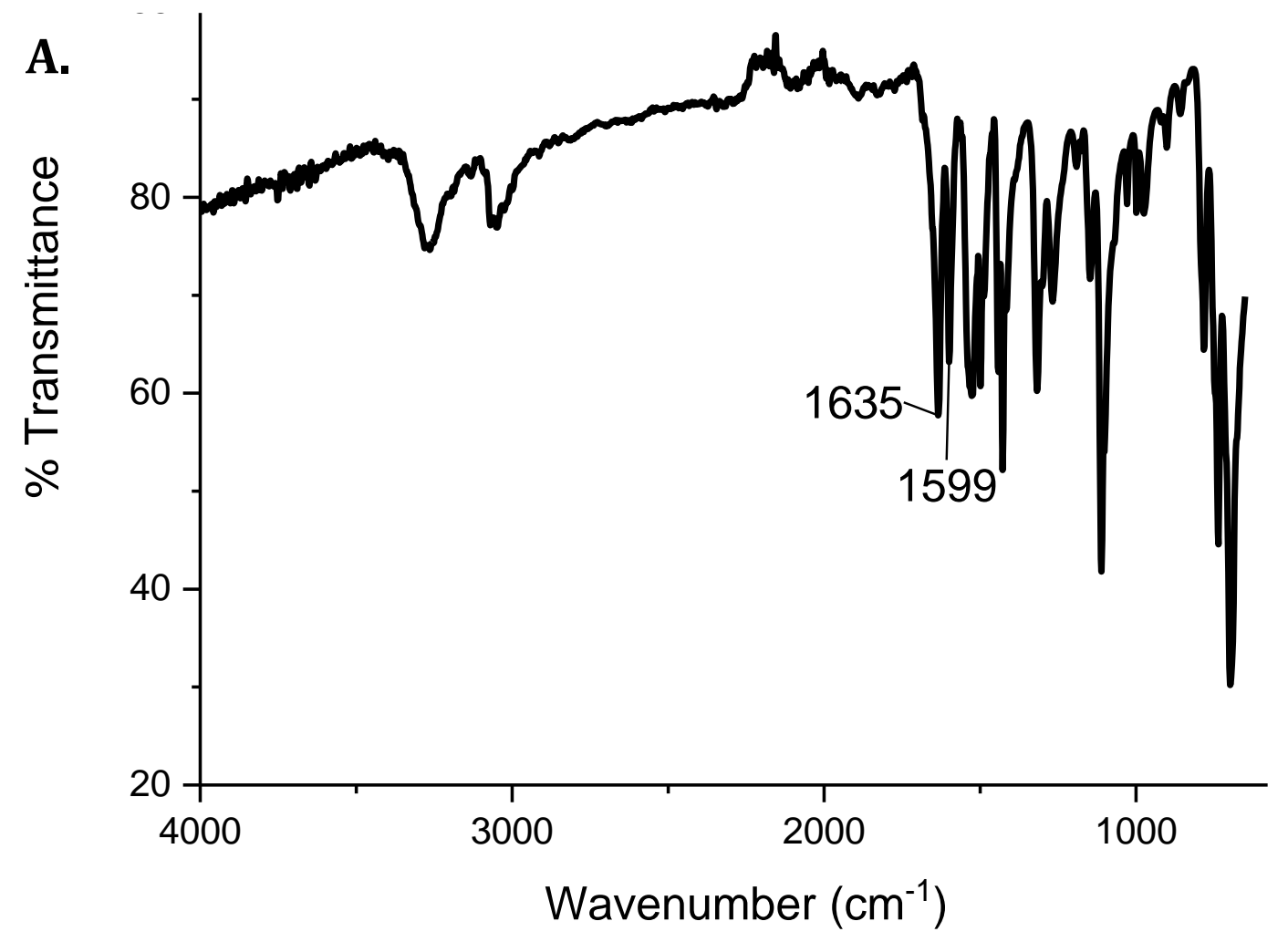




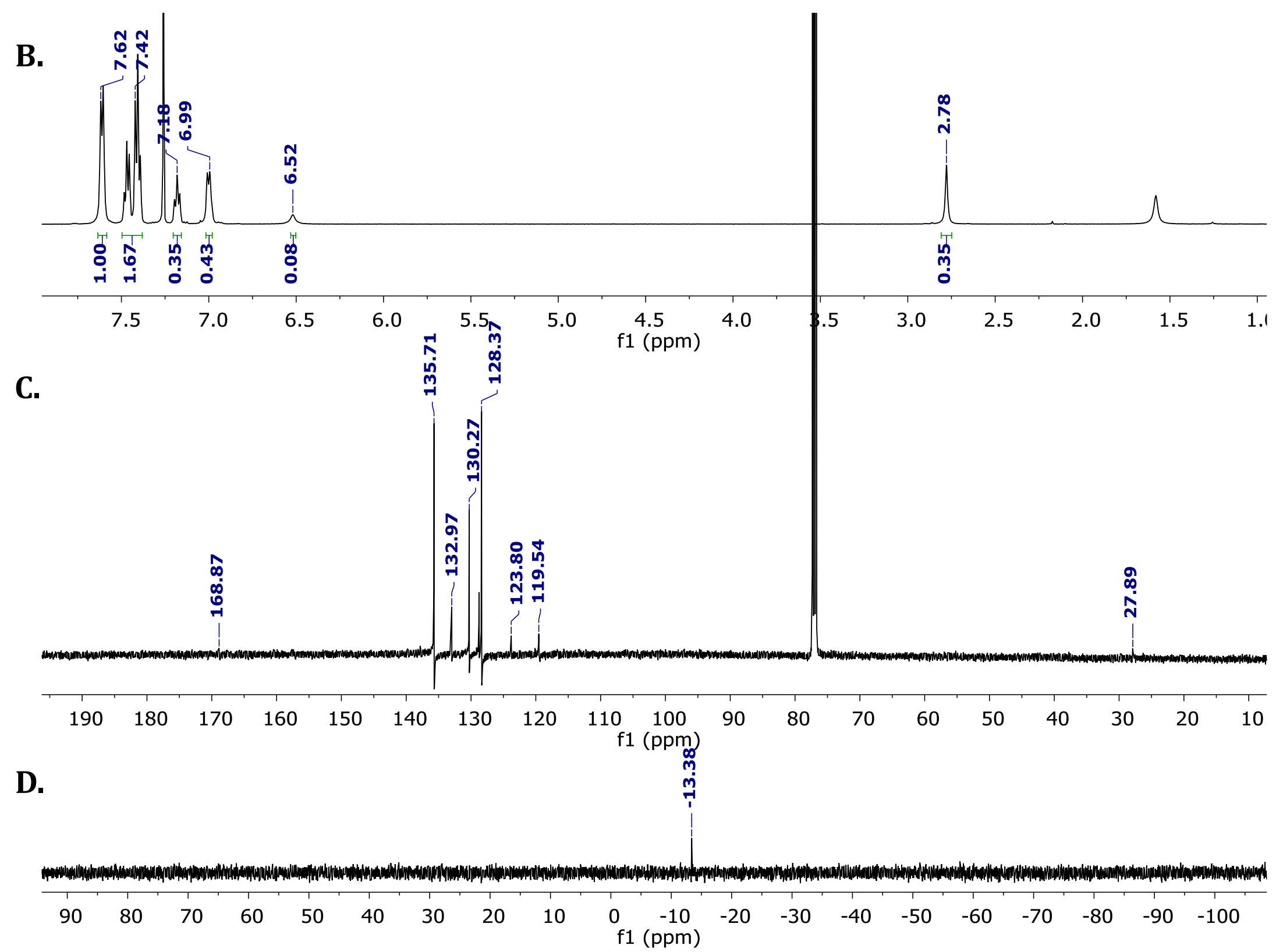


E. 09241920 \#101-118 RT: $0.45-0.53 \quad$ AV: 18 SB: 15 0.12-0.18 NL: 4.03E7
T: FTMS + p ESIFull ms [50.0000-600.0000]

100

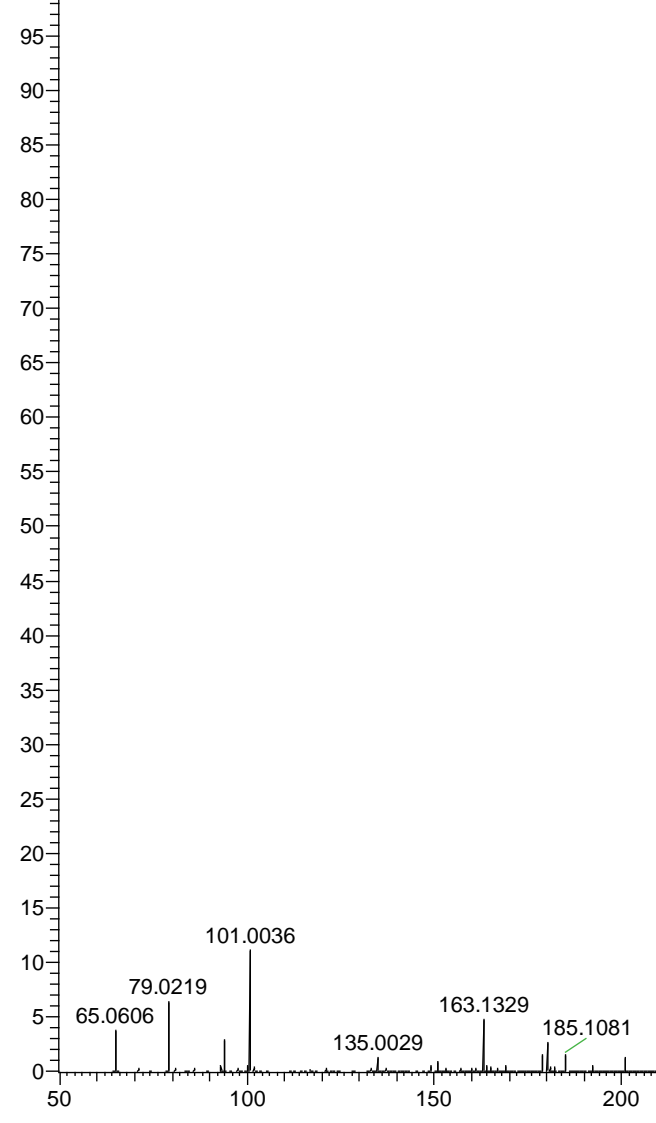

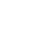

416.1437

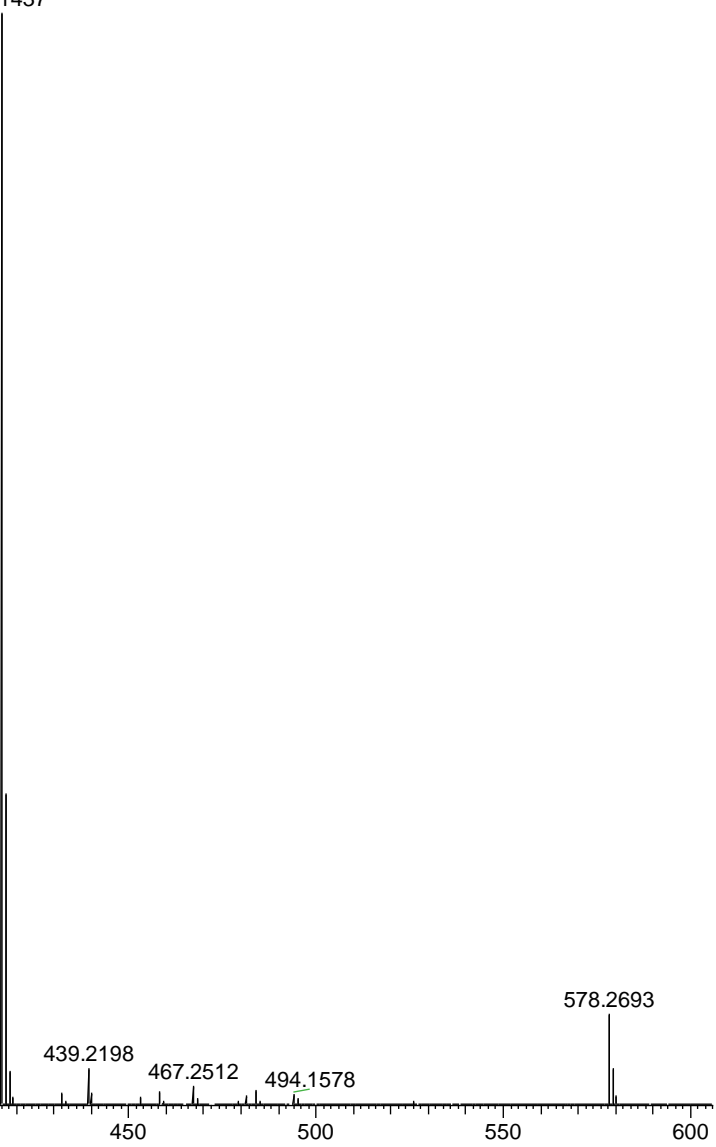

